\title{
Apar-T: code, validation, and physical interpretation of particle-in-cell results
}

\author{
Mickaël Melzani ${ }^{1}$, Christophe Winisdoerffer ${ }^{1}$, Rolf Walder ${ }^{1}$, Doris Folini ${ }^{1}$, Jean M. Favre ${ }^{2}$, \\ Stefan Krastanov ${ }^{1}$, and Peter Messmer ${ }^{3}$
}

\author{
1 École Normale Supérieure, CRAL, UMR CNRS 5574, Université de Lyon, Lyon, France \\ e-mail: mickael.melzani@ens-lyon.fr \\ 2 CSCS Lugano, Switzerland \\ ${ }^{3}$ NVIDIA Switzerland, Technoparkstr 1, 8005 Zürich, Switzerland
}

Received 23 March 2013 / Accepted 25 July 2013

\section{ABSTRACT}

\begin{abstract}
We present the parallel particle-in-cell (PIC) code Apar-T and, more importantly, address the fundamental question of the relations between the PIC model, the Vlasov-Maxwell theory, and real plasmas. First, we present four validation tests: spectra from simulations of thermal plasmas, linear growth rates of the relativistic tearing instability and of the filamentation instability, and nonlinear filamentation merging phase. For the filamentation instability we show that the effective growth rates measured on the total energy can differ by more than $50 \%$ from the linear cold predictions and from the fastest modes of the simulation. We link these discrepancies to the superparticle number per cell and to the level of field fluctuations. Second, we detail a new method for initial loading of Maxwell-Jüttner particle distributions with relativistic bulk velocity and relativistic temperature, and explain why the traditional method with individual particle boosting fails. The formulation of the relativistic Harris equilibrium is generalized to arbitrary temperature and mass ratios. Both are required for the tearing instability setup. Third, we turn to the key point of this paper and scrutinize the question of what description of (weakly coupled) physical plasmas is obtained by PIC models. These models rely on two building blocks: coarse-graining, i.e., grouping of the order of $p \sim 10^{10}$ real particles into a single computer superparticle, and field storage on a grid with its subsequent finite superparticle size. We introduce the notion of coarse-graining dependent quantities, i.e., quantities depending on $p$. They derive from the PIC plasma parameter $\Lambda^{\mathrm{PIC}}$, which we show to behave as $\Lambda^{\mathrm{PIC}} \propto 1 / p$. We explore two important implications. One is that PIC collision- and fluctuation-induced thermalization times are expected to scale with the number of superparticles per grid cell, and thus to be a factor $p \sim 10^{10}$ smaller than in real plasmas, a fact that we confirm with simulations. The other is that the level of electric field fluctuations scales as $1 / \Lambda^{\mathrm{PIC}} \propto p$. We provide a corresponding exact expression, taking into account the finite superparticle size. We confirm both expectations with simulations. Fourth, we compare the Vlasov-Maxwell theory, often used for code benchmarking, to the PIC model. The former describes a phase-space fluid with $\Lambda=+\infty$ and no correlations, while the PIC plasma features a small $\Lambda$ and a high level of correlations when compared to a real plasma. These differences have to be kept in mind when interpreting and validating PIC results against the Vlasov-Maxwell theory and when modeling real physical plasmas.
\end{abstract}

Key words. plasmas - methods: numerical - relativistic processes - instabilities - magnetic reconnection

\section{Introduction}

Full particle codes are now used by a large number of groups worldwide to study plasmas out of equilibrium in a large variety of environments, including electronic devices, inertial fusion (Dieckmann et al. 2006; Bret et al. 2010), tokamaks, Earth and solar magnetospheres (Hesse et al. 2001; Daughton et al. 2006; Drake et al. 2006; Klimas et al. 2008; Markidis et al. 2012; Baumann \& Nordlund 2012), or high-energy astrophysics (Silva et al. 2003; Pétri \& Lyubarsky 2007; Zenitani \& Hoshino 2008; Cerutti et al. 2012; Jaroschek et al. 2005; Nishikawa et al. 2008; Sironi \& Spitkovsky 2011).

Particle simulations actually appeared along with the first computers at universities and in industry around 1950. They first concerned electron beams in vacuum tubes, a device extensively used in the computers themselves. The beams were cold (Hartree 1950) or later hot (Tien \& Moshman 1956), and consisted of roughly 300 electron slabs moving in one dimension.

The step to plasma simulations was taken by Buneman (1959). He simulated an electrostatic plasma of 512 ions and electrons in one dimension, and showed that particle codes could be used to study the linear, nonlinear, and saturation phases of instabilities. At the time, the relevance of simulations with so few particles per Debye sphere was not clear and in 1962 Dawson (1962) and Eldridge \& Feix (1962) made an important contribution by showing that correct thermal behavior was produced.

All these algorithms used particle-particle interactions, and the first particle-mesh codes to introduce a grid appeared only later (Burger 1965; Hockney 1966; Yu et al. 1965). A great deal of literature on the drawbacks and benefits of the grid then appeared, and is now mostly concentrated in the two reference books of Birsdall \& Langdon (1985) and Hockney \& Eastwood (1988). Refinements of the algorithms quickly appeared (higher order grid interpolation, quiet codes, etc.), as well as code optimizations, at a time when programs were written in assembly language and depended heavily on machine architecture. Fully electromagnetic, relativistic, and 3D codes appeared with the studies of laser induced fusion (Buneman 1976), and closely resemble today's codes.

In 1967, it was possible for Birdsall (1967) to list the papers concerning simulations, and he noticed that they had grown exponentially since 1956 . In 1956, "many particles" meant 300; 
in $1985,10^{6}$; in $2008,10^{9}$; and in $2012,10^{12}$. For more historical details on PIC simulations, one may consult Birdsall (1967, 1999), Hockney \& Eastwood (1988, Sect. 9.1), or the introduction of Birsdall \& Langdon (1985).

Today, the latest generation of PIC algorithms consists of large versatile codes, featuring high order integration schemes and efficient parallelization. We can quote codes such as TRISTAN-MP (Spitkovsky 2005), OSIRIS (Fonseca et al. 2002, 2008), VORPAL (Nieter \& Cary 2004), WARP (Grote et al. 2005), ALaDyn (Benedetti et al. 2008), iPIC3D (Markidis et al. 2010), Photon-Plasma (Haugboelle et al. 2013), or Zeltron (Cerutti et al. 2013). They employ various simulation methods. The equations solved can differ: electrostatic codes, electromagnetic codes, Darwin approximation (Huang \& Ma 2008). The integration scheme can also vary: integration directly on the fields staggered on a grid, either with a charge conserving scheme or via solution of Poisson equation (Cerutti et al. 2012); or integration of the potential and correction of the discrepancies to charge conservation (Daughton et al. 2006). The time integration can be explicit or implicit (Markidis et al. 2012). Special parts of the numerical scheme can also differ; for example, the order of the field integration or the use of Fourier transforms. The interpolation of particle quantities to grid points and reciprocally can be done by a nearest grid point method (NGP), by a linear weighting (cloud in cell (CIC), or the PIC algorithm in the old terminology), or by a smoother shape (spline interpolation, Esirkepov 2001).

This article documents a new PIC code, Apar-T. Its first version, Tristan, was written by O. Buneman in 1990 (Matsumoto \& Omura 1993). It was then made parallel by Messmer (2001), and used in Messmer (2002) or Paesold et al. (2005). Given the large quantity of simulation methods, it is useful to first detail our algorithm. This is done in Sect. 2 and Appendix A.

In Sect. 3, we present a set of test problems and the results obtained with Apar-T. The first test is the study of the fluctuation spectra of a thermal plasma. The second and third tests explore the linear and nonlinear stages of the filamentation instability. A last test is the computation of the linear growth rates of the relativistic tearing instability, for which we give the general equilibrium relations for the relativistic Harris configuration with arbitrary temperature and mass ratio between the two species (Appendix C). In Sect. 4 we describe a method for loading a Maxwell-Jüttner momentum distribution with an arbitrary bulk velocity and temperature. The naive method, which initializes the comobile distribution and then boosts particles individually, is shown to be incorrect, mainly because space contraction is absent from the PIC code.

In Sect. 5, motivated by the above tests, we explore the physical implications of two main differences between PIC plasmas and real plasmas: coarse-graining, i.e., a reduction in the number of particles by a factor reaching $p \sim 10^{10}$, and the representation of the fields on a grid. We introduce the notion of coarse-graining dependent quantities, i.e., physical parameters depending on the number of real particles represented by a single computer superparticle. The prototype of these quantities is the plasma parameter $\Lambda$, which scales for a PIC plasma as $1 / p$. The dependence on coarse-graining has important consequences for key physical quantities such as the thermalization time, the collision time, or the level of field fluctuations.

We point out more generally that a PIC code simulates a microstate constituted by a restricted number of finite-sized particles each representing up to $10^{10}$ real plasma particles, while the Vlasov-Maxwell system models a plasma macrostate described by a continuous fluid in six-dimensional phase-space. These two descriptions are not equivalent and in particular PIC systems, with their small numbers of particles per Debye sphere, suffer from abnormally high noise levels and include to an unknown degree particle correlations absent from Vlasov-Maxwell equations.

We conclude in Sect. 6, stressing that validation and interpretation of PIC simulations requires detailed knowledge of the code as presented here as well as awareness of the above differences between the PIC model, the Vlasov-Maxwell model, and a real plasma.

\section{Physical model and numerical implementation}

This section presents the numerical scheme used in Apar-T. Broadly speaking, Apar-T is a parallel electromagnetic relativistic 3D PIC code with a staggered grid, where the fields are integrated via Faraday and Maxwell-Ampère equations, currents are computed by charge conserving volume weighting (CIC), and fields are interpolated with the same CIC volume weighting method.

\subsection{The PIC plasma}

The code simulates the time-evolution of charged particles under the action of the electromagnetic fields that they generate, and the evolution of these fields.

Plasmas in nature contain millions to tens of billions of particles per Debye sphere, and relevant microphysical phenomena spread over numerous Debye lengths. It is impossible to track these particles one by one. Rather, the numerical particles represent numerous real particles, and are consequently called superparticles.

A superparticle represents either $p$ real ions (having then a rest mass $m_{\mathrm{sp}}=p \times m_{\mathrm{i}}$ and a charge $\left.q_{\mathrm{sp}}=p \times q_{\mathrm{i}}\right)$, or $p$ real electrons (having then a rest mass $m_{\mathrm{sp}}=p \times m_{\mathrm{e}}$ and a charge $\left.q_{\mathrm{sp}}=p \times q_{\mathrm{e}}\right)$. The ratio of ion to electron charge is always $q_{\mathrm{i}} / q_{\mathrm{e}}=-1$, while that of their rest masses $m_{\mathrm{i}} / m_{\mathrm{e}}$ can be freely specified. Pair plasmas can thus be simulated. In Apar-T the number of real particles per superparticles $p$ is the same for all superparticles at all times, but other codes can introduce superparticle splitting (Fujimoto \& Machida 2006; Haugboelle et al. 2013; Cerutti et al. 2013).

We denote the physical size of a grid cell by $X_{0}$, a reference number of superparticles per cell by $\rho_{\mathrm{sp}}^{0}$ (including both ion superparticles and electron superparticles), and its associated number density of electrons by $n_{\mathrm{e}}^{0}$. Initially, the plasma is assumed to be quasi-neutral, in the sense that we load the same number of ion superparticles and electron superparticles in each cell. We have the relation

$2 n_{\mathrm{e}}^{0} \times X_{0}^{3}=$ number of real particles in one cell $=p \times \rho_{\mathrm{sp}}^{0}$.

The equations governing the superparticle plasma are the equation of motion with the Lorentz force for each superparticle, and Maxwell equations coupled to the superparticle motions by the current:

$$
\begin{aligned}
& \frac{\mathrm{d}}{\mathrm{d} t}\left(\gamma_{\mathrm{sp}} \boldsymbol{v}_{\mathrm{sp}}\right)=\frac{q_{\mathrm{sp}}}{m_{\mathrm{sp}}}\left(\boldsymbol{e}+\frac{\boldsymbol{v}_{\mathrm{sp}}}{c} \wedge \boldsymbol{b}\right) \\
& \frac{\mathrm{d}}{\mathrm{d} t} \boldsymbol{x}_{\mathrm{sp}}=\boldsymbol{v}_{\mathrm{sp}} \\
& \frac{\partial \boldsymbol{b}}{\partial t}=-c \nabla \wedge \boldsymbol{e} \\
& \frac{\partial \boldsymbol{e}}{\partial t}=c \nabla \wedge \boldsymbol{b}-\frac{1}{\epsilon_{0}} \boldsymbol{j} \\
& \boldsymbol{j}=\sum_{\mathrm{sp}} q_{\mathrm{sp}} \boldsymbol{v}_{\mathrm{sp}} S\left(\boldsymbol{x}-\boldsymbol{x}_{\mathrm{sp}}\right)
\end{aligned}
$$


Here, $c$ is the speed of light, $\boldsymbol{e}$ the electric field, $\boldsymbol{b}=c \boldsymbol{B}$ is $c$ times the magnetic field; $q_{\mathrm{sp}}, m_{\mathrm{sp}}, \gamma_{\mathrm{sp}}, \boldsymbol{v}_{\mathrm{sp}}$, and $\boldsymbol{x}_{\mathrm{sp}}$ are the charge, mass, Lorentz factor, velocity, and position of the superparticle number sp. The fields are stored on a grid, and a consequence is that the superparticles are seen by the grid as having a finite shape $S$, linked to the interpolation scheme used in the code.

In Apar-T we do not integrate the two other Maxwell equations because if they hold initially, then fulfilling the equation of conservation of charge and Eqs. (2c) and (2d) at all times insures that they remain correct to round-off errors. That the current is indeed computed in a charge conserving way is detailed in Appendix A.1. Initially, the fields and the charge density are correctly built by setting a magnetic field satisfying $\nabla \wedge \boldsymbol{b}=\mu_{0} \boldsymbol{j}$, a null electric field, and by placing the superparticles by pairs with one ion superparticle and one electron superparticle on top of each other so that the charge density is zero.

\subsection{Numerical implementation of Apar-T}

The code is based on the PIC program Tristan written by Buneman (Matsumoto \& Omura 1993), parallelized by Messmer (2001). We largely modified its structure, that now uses Fortran modules and allows for more flexibility, including the possibility of switching between different initial conditions or boundaries by changing entries in a configuration textfile.

The need for a deep understanding of simulation methods to interpret their results, as is the case for the test problems of Sect. 3, motivated a detailed description of Apar-T. This section presents important points. More details (numerical scheme and parallelization efficiency) can be found in Appendix A.

Briefly, the global integration scheme of Eqs. (2a)-(2e) is a time-centered and time-reversible leap-frog scheme, and is second-order accurate in time and space. The electric and magnetic fields are stored on a staggered Yee lattice, which allows for a simple integration explicit in time of Eqs. (2c) and (2d) (without the current). The current is computed with the volume change of the superparticles in the grid cells, and added in a time explicit way to the integration of the electric field (Eq. (2d)).

\subsubsection{Temporal and spatial discretization, normalization}

The spatial discretization of the code is $X_{0}=d_{\mathrm{e}}^{0} / n_{x}$, a fraction $n_{x}$ of the electron skin depth $d_{\mathrm{e}}^{0}=c / \omega_{\mathrm{pe}}^{0}$, where the electron plasma pulsation is $\omega_{\mathrm{pe}}^{0}=\sqrt{n_{\mathrm{e}}^{0} e^{2} /\left(\epsilon_{0} m_{\mathrm{e}}\right)}$, with $-e$ and $m_{\mathrm{e}}$ the electron charge and rest mass. The timestep $\Delta t$ is a fraction $n_{t}$ of the electron plasma period: $\Delta t=T_{\mathrm{pe}}^{0} / n_{t}$, with $T_{\mathrm{pe}}^{0}=2 \pi / \omega_{\mathrm{pe}}^{0}$. We stress that the superscript ${ }^{0}$ is used for quantities based on the reference density $n_{\mathrm{e}}^{0}$.

Spatial quantities are normalized by the cell length $X_{0}$, and normalized quantities are then denoted with a tilde. For example, the electron Debye length $\lambda_{\mathrm{De}}^{0}=\sqrt{\epsilon_{0} T_{\mathrm{e}} /\left(n_{\mathrm{e}}^{0} e^{2}\right)}=v_{\mathrm{th}, \mathrm{e}} / \omega_{\mathrm{pe}}^{0}$ (with $\left.v_{\text {th,e }}=\sqrt{T_{\mathrm{e}} / m_{\mathrm{e}}}\right)$ has for normalized counterpart $\tilde{\lambda}_{\mathrm{De}}^{0}=\lambda_{\mathrm{De}}^{0} / X_{0}=$ $n_{x} v_{\text {th,e }} / c$.

\subsubsection{Superparticle volume}

The use of a grid for PIC algorithms implies that the fields are known at grid nodes, and that information relative to the superparticles (charge and current) need to be interpolated on the grid. This interpolation is equivalent to considering the superparticles as clouds of charge of finite extension (Langdon 1970). The shape of the cloud then determines the interpolation formula.
In our case, a superparticle is assumed to be a cube of volume $V_{\mathrm{sp}}=X_{0}^{3}$, and the current it produces is calculated by the change of the volume of the superparticle in the cell containing its center. This interpolation scheme is equivalent to linear weighting (CIC) and is exactly charge conserving. Details of the numerical implementation of the current computation can be found in Appendix A; in Sect. 5 the implications of the superparticle finite sizes are discussed.

\subsubsection{Particle initialization in momentum space}

Several momentum distributions can be loaded: MaxwellBoltzmann distribution with anisotropic temperature, boosted Maxwell-Boltzmann distribution, waterbag distribution, or Maxwell-Jüttner distribution. We have not found in the literature a method for initializing the Maxwell-Jüttner distribution when both the bulk velocity and the temperature are relativistic, so we present one in Sect. 4.

\subsubsection{Input, output, and data analysis}

Data output and data analysis can be very time consuming for large scale simulations. To reduce data storage and writing time, we implemented parallel output in HDF5 format ${ }^{1}$. Files are written according to the .h5part format, and can be read by the advanced visualization and data-analysis software VisIt (Childs et al. 2012), which is fully parallel, but also by a reader in Python. The .h5part files can either contain the whole simulation data, and then be used to restart the simulation, or be lighter with only a fraction of the particles. These lighter files then include cell-averaged quantities related to the particles, such as the mean particle velocity or number, temperature, highest energy, etc.

We implemented the VisIt Libsim in situ library (Whitlock et al. 2011) into Apar-T. In this way, VisIt is able to connect to the simulation while it is running and to access the solver's data at the current timestep. It can then perform data visualization and data analysis without the need to write data on the hard drive. This feature is fully parallelized, by exploiting the datadistribution model of the code, and as such is not restricted to the current parallelization model. It allows the data-IO from memory to hard drive - which is a major reason for slow-down of simulations using big data-sets - to be significantly reduced. For example, a volume-rendering of 3D data performed in situ takes less time than dumping $10 \mathrm{~GB}$ of data to the disk. VisIt in situ is also well suited to monitor ongoing simulations and to singlestep through the execution and is, in this way, of great help for debugging.

Finally, a set of test problems has been implemented. Based on a Python script, these problems can be automatically run to check code sanity after modifications.

\section{Examples and code validation}

\subsection{Cold plasma modes}

A first test is to simulate a thermal plasma at rest and to observe its modes of oscillation. It is an easy test if we focus on the pulsations for modes of zero wavevector, $\boldsymbol{k}=0$. To do so, we compute at each timestep $t_{j}$ the sum of the momentum of the particles along a given direction, for example the $x$-direction, $\sum_{\mathrm{sp}} \gamma_{\mathrm{sp}}\left(t_{j}\right) v_{\mathrm{sp}, x}\left(t_{j}\right)$, where the summation runs over

1 http://www.hdfgroup.org/HDF5/ 
all the electron superparticles in the simulation (of Lorentz factor $\gamma_{\mathrm{sp}}$ and velocity $v_{\mathrm{sp}}$ ). This sum is equivalent to the volume integral of the momentum, and is thus equal to the $\boldsymbol{k}=0$ Fourier mode of the electron momentum, with a spectral resolution of $2 \pi /$ [box size in units of electron skin depth]. We then perform a Fourier transform in time to extract the pulsations of oscillation,

$F_{x}(\omega)=\sum_{j} \exp \left(\mathrm{i} \omega t_{j}\right) \times \sum_{\mathrm{sp}} \gamma_{\mathrm{sp}}\left(t_{j}\right) v_{\mathrm{sp}, x}\left(t_{j}\right)$,

and similarly along $y$ and $z$.

For all the simulations of this section, the initial state consists of a homogeneous plasma at rest, with superparticles loaded according to a classical Maxwell-Boltzmann distribution. A uniform background magnetic field along $z$ is set up for the magnetized plasma case. Periodic boundaries are used.

When the background magnetic field $\boldsymbol{B}_{0}$ is strong (electron cyclotron pulsation larger than electron plasma pulsation, $\omega_{\mathrm{ce}} \gg$ $\omega_{\text {pe }}$ ), the particle trajectories are Larmor gyrations in $\boldsymbol{B}_{0}$, unperturbed by collective effects such as Langmuir oscillations. This example then probes the accuracy of the particle motion integrator. When the background magnetic field is weaker $\left(\omega_{\mathrm{ce}} \lesssim \omega_{\text {pe }}\right)$, the dynamics is set by Langmuir oscillations possibly modified by $\boldsymbol{B}_{0}$. These oscillations involve the creation of electric fields by local charge imbalance. The fields set the particles into motion, and the particles then oscillate because of their finite inertia. Several parts of the algorithm are thus involved: electric field production and propagation, as well as particle motion.

\subsubsection{No background magnetic field}

With no background magnetic field, the only cold modes are the electromagnetic transverse wave of dispersion relation $\omega_{\mathrm{Tr}}^{2}=$ $\omega_{\mathrm{P}}^{2}\left[1+\left(k c / \omega_{\mathrm{P}}\right)^{2}\right]$ and the electrostatic Langmuir oscillation at the plasma frequency $\omega_{\mathrm{P}}=\omega_{\mathrm{pe}}\left(1+m_{\mathrm{e}} / m_{\mathrm{i}}\right)^{1 / 2}$. The latter is modified by thermal effects to a wave of dispersion relation $\omega_{\mathrm{La}}^{2}=\omega_{\mathrm{P}}^{2}\left(1+3 k^{2} \lambda_{\mathrm{D}}^{2}\right)$. Consequently, we expect $F_{a}(\omega)$ to peak at $\omega_{\mathrm{La}}(\boldsymbol{k}=0)=\omega_{\mathrm{Tr}}(\boldsymbol{k}=0)=\omega_{\mathrm{P}}$.

Our simulations span a large range of parameters: $n_{x}$ and $n_{t}$ (spatial and temporal resolution, see Sect. 2.2.1) vary between 5 and 50 and between 300 and 2000, respectively; $\rho_{\mathrm{sp}}$ (the number of superparticles per cell) varies between 4 and 32 ; and the thermal velocity $v_{\mathrm{th}, \mathrm{e}}=\sqrt{T_{\mathrm{e}} / m_{\mathrm{e}}}$ of the electrons between $0.04 c$ and $0.1 c$ (the ions have the same temperature as the electrons). This results in Debye lengths between 0.2 and 2 cells. We checked that the simulation box size, comprised between 10 and 30 Debye lengths, does not influence the results.

We use a mass ratio $m_{\mathrm{i}} / m_{\mathrm{e}}=49$, which results in $\omega_{\mathrm{P}}=$ $1.010 \omega_{\mathrm{pe}}$. Our simulations last $100 T_{\mathrm{pe}}$, so that the frequency resolution is $\Delta \omega=2 \pi /\left(100 T_{\mathrm{pe}}\right)=0.01 \omega_{\mathrm{pe}}$.

For all these parameters, we find that the position and width of the frequency peak are always the same as in Fig. 1. It coincides with the theoretical plasma pulsation, which is expected because our temporal spectra are for the wavenumber $\boldsymbol{k}=0$, and because $\omega(\boldsymbol{k}=0)=\omega_{\mathrm{P}}$ for the two modes present in this situation.

This is the case even for simulations where the Debye length is not resolved. However, an under-resolved Debye length leads to more numerical heating and can trigger instabilities in situations less trivial than a thermal plasma at rest (see Appendix B), so that we have not pushed our investigations too far in this direction.

The main difference between the simulations is that less resolved ones present noisier spectra, and thus more fluctuations.

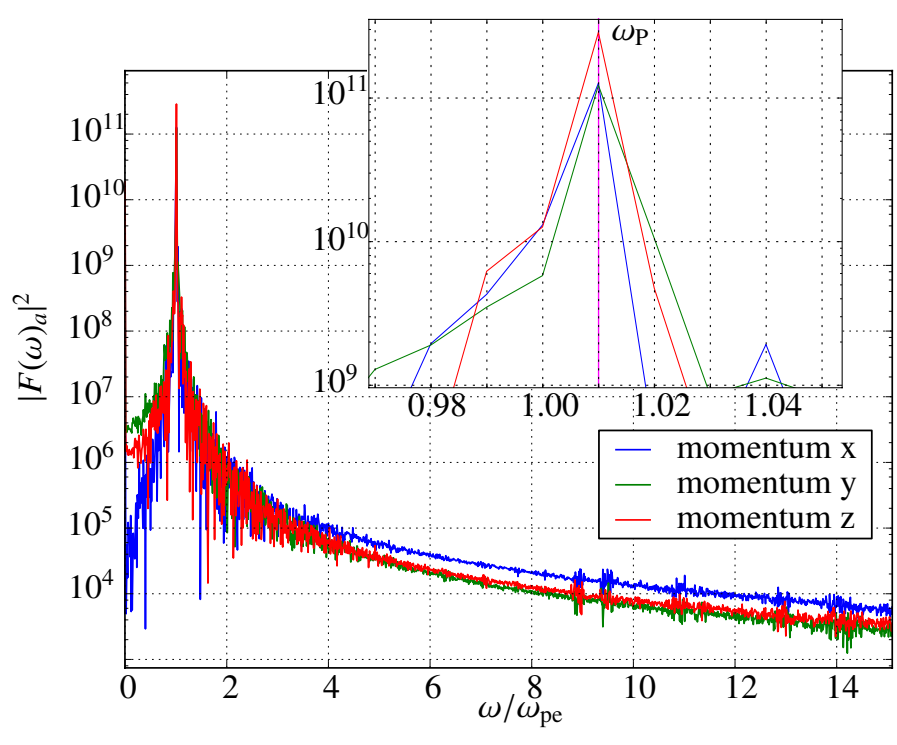

Fig. 1. Power spectra of the total momentum of the electrons $\left|F_{a}\right|^{2}$ (Eq. (3)) with $a=x, y$, or $z$ in the unmagnetized case. The inset is a zoom around the peak. Here $n_{x}=25, n_{t}=500, \rho_{\mathrm{sp}}=16, v_{\mathrm{th}, \mathrm{e}}=0.04 c$, $T_{\mathrm{i}}=T_{\mathrm{e}}$, box size of $25 \times 25 \times 25$ cells, duration of $100 T_{\mathrm{pe}}$.

The increase of fluctuation level with decreasing resolution is a universal feature of PIC simulations and is explored in more detail in Sect. 5.

\subsubsection{With a background magnetic field}

In a uniform and cold magnetized plasma, the plasma modes depend solely on the ratio of the electron cyclotron pulsation $\omega_{\mathrm{ce}}=e B / m_{\mathrm{e}}$ to the electron plasma pulsation $\omega_{\mathrm{pe}}$ (for a fixed $\left.m_{\mathrm{i}} / m_{\mathrm{e}}\right)$. This ratio sets the relative importance of individual particle motion $\left(\omega_{\text {ce }}\right)$ against collective effects $\left(\omega_{\text {pe }}\right)$. In addition, the background magnetic field favors a direction, thus making the mode spectrum anisotropic (however, the mode pulsations for $\boldsymbol{k}=0$ remain independent of the direction of the wavevector).

For wavevectors parallel to the magnetic field, the Langmuir oscillation remains unchanged because the oscillations of the particles are longitudinal, and thus along $\boldsymbol{B}_{0}$ and unaffected by the magnetic field. On the other hand, the electromagnetic wave with $\boldsymbol{k}$ along $\boldsymbol{B}_{0}$ separates into two branches, one starting at $\omega_{k=0}=\omega_{\mathrm{L}}=0.5\left[\left(\omega_{\mathrm{ce}}^{2}+4 \omega_{\mathrm{pe}}^{2}\right)^{1 / 2}-\omega_{\mathrm{ce}}\right]$ and the other at $\omega_{k=0}=\omega_{\mathrm{R}}=0.5\left[\left(\omega_{\mathrm{ce}}^{2}+4 \omega_{\mathrm{pe}}^{2}\right)^{1 / 2}+\omega_{\mathrm{ce}}\right]$ (see, e.g., Fitzpatrick 2011, chap. 4), in both cases with particles oscillating in the transverse plane, i.e., perpendicular to $\boldsymbol{B}_{0}$. Two other branches appear, but they start at $\omega_{k=0}=0$ and will thus not appear in $F_{a}(\omega)$.

For wavevectors perpendicular to the magnetic field, the presence of the background magnetic field deforms the Langmuir oscillation $\omega(k)=\omega_{\mathrm{P}}$ into a branch starting from $\omega_{k=0}=\omega_{\mathrm{L}}$, with particle oscillations in the plane perpendicular to $\boldsymbol{B}_{0}$. The transverse electromagnetic wave still exists with particle oscillations along $\boldsymbol{B}_{0}$, unaffected by the magnetic field. Another branch appears, which is a deformation of the transverse electromagnetic wave for particle oscillations not along $\boldsymbol{B}_{0}$, and starts at $\omega_{k=0}=\omega_{\mathrm{R}}$ with oscillations in the plane perpendicular to $\boldsymbol{B}_{0}$. Another branch appears, starting at $\omega_{\boldsymbol{k}=0}=0$.

All in all, we expect to find a peak at $\omega=\omega_{\mathrm{P}}$ for the component of the momentum parallel to $\boldsymbol{B}_{0}$, and two peaks at $\omega=\omega_{\mathrm{L}}$ and $\omega_{\mathrm{R}}$ for the component of the momentum perpendicular 
M. Melzani et al.: Apar-T: code, validation, and physical interpretation of particle-in-cell results

Table 1. Theoretical versus experimental pulsations for a magnetized cold plasma.

\begin{tabular}{|c|c|c|c|c|c|c|c|c|c|c|c|c|}
\hline$\omega_{\mathrm{ce}}$ & $n_{t}$ & $n_{x}$ & $\rho_{\mathrm{sp}}$ & $v_{\text {th, }} / c$ & $\tilde{\lambda}_{\mathrm{De}}$ & $\tilde{r}_{\mathrm{ce}}$ & $\omega_{\mathrm{ce}} \Delta t$ & $\omega_{/ /}^{\text {simu }}$ & $\omega_{\perp}^{\text {simu }}$ & $\omega_{\mathrm{P}}^{\text {theory }}$ & $\omega_{\mathrm{L}}^{\text {theory }}$ & $\omega_{\mathrm{R}}^{\text {theory }}$ \\
\hline $\begin{array}{l}0.5 \\
\prime \prime\end{array}$ & $\begin{array}{c}4000 \\
\prime \prime\end{array}$ & $\begin{array}{l}32 \\
\prime \prime\end{array}$ & $\begin{array}{c}4 \\
16\end{array}$ & $\begin{array}{c}0.04 \\
\prime \prime\end{array}$ & $\begin{array}{c}1.28 \\
\prime \prime\end{array}$ & $\begin{array}{c}2.56 \\
\prime \prime\end{array}$ & $7.9 \times 10^{\prime \prime}$ & $\begin{array}{l}1.01 \\
1.01\end{array}$ & $\begin{array}{l}0.79,1.28 \\
0.79,1.28\end{array}$ & $\begin{array}{c}1.010 \\
\prime \prime\end{array}$ & $\begin{array}{c}0.78 \\
\prime \prime\end{array}$ & $\begin{array}{c}1.28 \\
\prime \prime\end{array}$ \\
\hline $\begin{array}{l}1 \\
\prime \prime\end{array}$ & $\begin{array}{l}1000 \\
4000\end{array}$ & $\begin{array}{l}25 \\
32\end{array}$ & $\begin{array}{c}16 \\
4\end{array}$ & " & $\begin{array}{c}1 \\
1.28\end{array}$ & $\begin{array}{c}1 \\
1.28\end{array}$ & $\begin{array}{l}6.3 \times 10^{-3} \\
1.6 \times 10^{-3}\end{array}$ & $\begin{array}{l}1.01 \\
1.01\end{array}$ & $\begin{array}{l}0.64,1.62 \\
0.64,1.62\end{array}$ & $\begin{array}{c}1.010 \\
\prime \prime\end{array}$ & $\begin{array}{c}0.62 \\
\prime \prime\end{array}$ & $\begin{array}{c}1.62 \text { Fig. } 2 \\
\text { " }\end{array}$ \\
\hline $\begin{array}{l}2 \\
\text { " } \\
\text { " } \\
\text { " }\end{array}$ & $\begin{array}{l}1000 \\
1000 \\
4000 \\
4000\end{array}$ & $\begin{array}{l}16 \\
25 \\
43 \\
64\end{array}$ & $\begin{array}{c}4 \\
16 \\
4 \\
4\end{array}$ & $\begin{array}{l}1 \\
\text { " } \\
\text { " } \\
\text { " }\end{array}$ & $\begin{array}{c}0.64 \\
1 \\
1.72 \\
2.56\end{array}$ & $\begin{array}{c}0.32 \\
0.5 \\
0.86 \\
1.28\end{array}$ & $\begin{array}{c}1.3 \times 10^{-2} \\
3.1 \times 10^{-3} \\
\quad 10^{-3}\end{array}$ & $\begin{array}{l}1.01 \\
1.01 \\
1.01 \\
1.01\end{array}$ & $\begin{array}{l}0.43,2.64 \\
0.46,2.40 \\
0.46,2.40 \\
0.46,2.40\end{array}$ & $\begin{array}{c}1.010 \\
\prime \prime \\
\prime \prime \\
\prime \prime\end{array}$ & $\begin{array}{l}0.41 \\
" 1 \\
\prime \prime \\
\prime \prime\end{array}$ & $\begin{array}{c}2.41 \\
\text { " } \\
\prime \prime \\
\prime \prime\end{array}$ \\
\hline $\begin{array}{l}4 \\
\prime \prime\end{array}$ & $\begin{array}{l}1000 \\
2000\end{array}$ & $\begin{array}{c}25 \\
128\end{array}$ & $\begin{array}{l}4 \\
4\end{array}$ & " & $\begin{array}{c}1 \\
5.12\end{array}$ & $\begin{array}{l}0.25 \\
1.28\end{array}$ & $\begin{array}{l}2.5 \times 10^{-2} \\
1.3 \times 10^{-2}\end{array}$ & $\begin{array}{l}1.01 \\
1.01\end{array}$ & $\begin{array}{l}0.32,4.22 \\
0.32,4.21\end{array}$ & 1.010 & 0.24 & 4.24 \\
\hline
\end{tabular}

Notes. $\omega_{\perp}^{\text {simu }}$ and $\omega_{/ /}^{\text {simu }}$ are the peak in the spectra of the parallel and perpendicular total particle momentum from the simulations. The right columns give the theoretical pulsations for a cold plasma. See the main text for the formula. Here $m_{\mathrm{i}} / m_{\mathrm{e}}=49$, for the expression of the plasma pulsation $\omega_{\mathrm{P}}$, and $m_{\mathrm{i}} / m_{\mathrm{e}}=\infty$ for the expressions of the right and left cut-off pulsations $\left(\omega_{\mathrm{R}}\right.$ and $\left.\omega_{\mathrm{L}}\right)$. The duration of each simulation is $100 T_{\mathrm{pe}}$, implying a spectral precision of $\Delta \omega=0.01 \omega_{\mathrm{pe}}$. Ion and electron temperatures are equal. $\tilde{\lambda}_{\mathrm{De}}$ and $\tilde{r}_{\mathrm{ce}}$ are the electron Debye length and Larmor radius in units of cell number. All pulsations are in units of $\omega_{\mathrm{pe}}$.

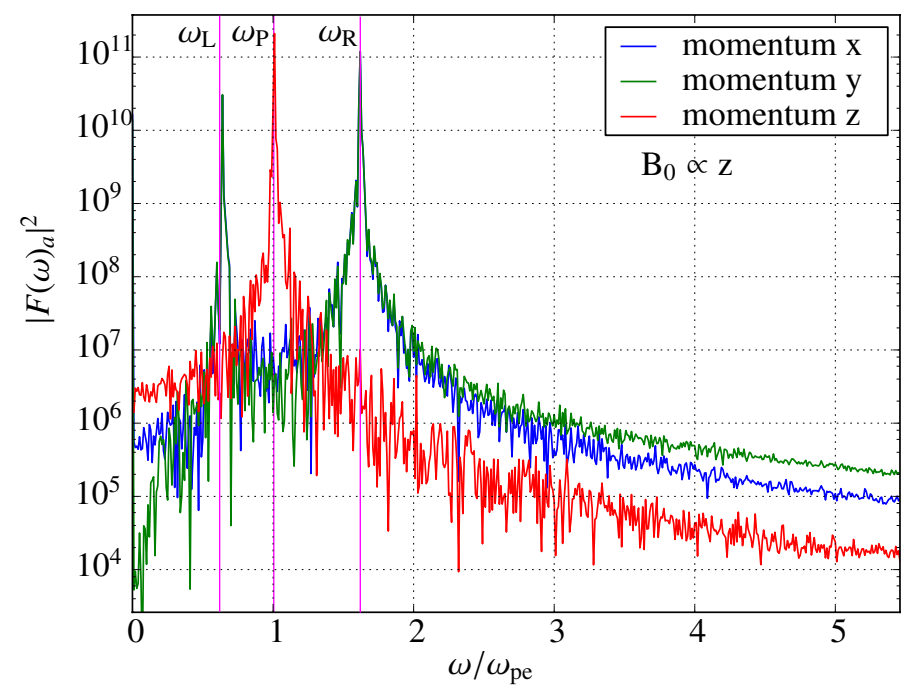

Fig. 2. Power spectra of the total momentum of the electrons $\left|F_{a}\right|^{2}$ (Eq. (3)) with $a=x, y$, or $z$ in the magnetized case. Parameters are given in Table 1 (case labeled Fig. 2). The purple lines are the pulsations $\omega_{\mathrm{L}}^{\text {theory }}, \omega_{\mathrm{P}}^{\text {theory }}$, and $\omega_{\mathrm{R}}^{\text {theory }}$.

to $\boldsymbol{B}_{0}$. We ran the set of simulations described in Table 1, with a ratio $\omega_{\text {ce }} / \omega_{\text {pe }}$ ranging from 0.5 to $4, n_{x}$ from 16 to $128, n_{t}$ from 1000 to 4000 , and $\rho_{\mathrm{sp}}$ from 4 to 16 , and we did find the required pulsation peaks for $F_{a}(\omega)$ (see Fig. 2 for a sample spectrum). The positions and widths of these three peaks are almost constant within our parameter range.

We note that the peak positions and widths are not changed even for cases where the thermal Larmor radius $\tilde{r}_{\mathrm{ce}}=r_{\mathrm{ce}} / X_{0}=$ $v_{\text {th,e }} /\left(X_{0} \omega_{\text {ce }}\right)=\tilde{\lambda}_{\text {De }} /\left(\omega_{\text {ce }} / \omega_{\text {pe }}\right)$ is not resolved. It is expected that the resolution of the Larmor radius by the grid is of no importance to describe particle trajectories in constant fields, because the interpolation of these fields from grid points to superparticle position gives the same result regardless of the grid size if the fields are constant. The relevant constraint is instead that $r_{\mathrm{ce}}$ should be resolved along the trajectory, $v_{\mathrm{sp}} \Delta t<r_{\mathrm{ce}}$, with $v_{\mathrm{sp}}$ the superparticle velocity. This relation is equivalent to $\Delta t<\omega_{\mathrm{ce}}^{-1}$.

However, for simulations with under-resolved Larmor radii the electric field energy starts to behave abnormally after some tens of plasma pulsations, and energy conservation curves
Table 2. Energy conservation for simulations of a thermal plasma with no background magnetic field.

\begin{tabular}{lccc|cc}
\hline \hline$n_{t}$ & $n_{x}$ & $\rho_{\text {sp }}$ & $v_{\text {th,e }} / c$ & $\tilde{\lambda}_{\text {De }}$ & $T_{\text {pe } \frac{\mathrm{d}}{\mathrm{d} t} \frac{E_{\text {tot }}(t)}{E_{\text {tot }}(0)}}$ \\
\hline 500 to 2000 & 10 & 16 & 0.04 & 0.4 & $1.6 \times 10^{-4}$ \\
$" \prime$ & 10 & 16 & 0.10 & 1 & $5.8 \times 10^{-5}$ \\
$"$ & 14 & 16 & 0.07 & 1 & $3.5 \times 10^{-5}$ \\
$"$ & 18 & 16 & 0.04 & 0.7 & $5.3 \times 10^{-5}$ \\
300 to 2000 & 25 & 4 & 0.04 & 1 & $1.0 \times 10^{-4}$ \\
$" \prime$ & 25 & 16 & 0.04 & 1 & $2.5 \times 10^{-5}$ \\
$"$ & 25 & 32 & 0.04 & 1 & $1.3 \times 10^{-5}$ \\
\hline
\end{tabular}

Notes. The energy increase rate is measured on the total energy normalized by the total initial energy, while time is again normalized with the electron plasma pulsation $T_{\mathrm{pe}}$. Here, $m_{\mathrm{i}} / m_{\mathrm{e}}=49$ and $T_{\mathrm{i}}=T_{\mathrm{e}}$.

present an exponential heating (see Sect. 3.1.3) that can lead to dramatic consequences. This parameter range must be avoided.

\subsubsection{Energy conservation}

Independent of the strength of the background magnetic field, we observe a linear increase of the total energy with time that is due to interactions of particles with the grid (see Appendix B.2). The growth rate is independent of the size of the timestep (from $n_{t}=2000$ down to Courant condition $\Delta t \sim X_{0} / c$, equivalent to $n_{t}=2 \pi n_{x}$ ). Its dependence on the number of superparticles per cell is quite precisely given by $1 / \rho_{\text {sp }}$. However, its dependence on the spatial resolution $n_{x}$ and thermal spread $v_{\text {th,e }}$ is less clear. In particular, it does not depend only on the product $n_{x} v_{\text {th,e }} / c=$ $\tilde{\lambda}_{\text {De }}$. The rate increases with increasing $v_{\text {th,e }}$, and decreases with increasing $n_{x}$. Some examples are given for reference in Table 2.

Simulations with under-resolved Larmor radii $\tilde{r}_{\text {ce }}<1$ show an exponential (instead of linear) increase of the total energy starting after roughly $40 T_{\mathrm{pe}}$. This numerical instability is believed to arise because field perturbation at wavelength $\lambda=2 r_{\text {ce }}$ and their aliases $\left( \pm \lambda+n \times 2 \pi / X_{0}\right.$, with $n$ an integer and $X_{0}$ the grid size) are allowed to couple when $2 X_{0}>\lambda$ (see Appendix B.2). An inspection of the energy curves shows that the energy gain is for the kinetic energy. We note that it did not disturbed the spectra of Sect. 3.1.2 because they were computed before the heating reached a significant level. It is interesting 
to note that this numerical instability is develops slowly, so that particles with under-resolved Larmor radii in constant fields can be included in simulations if they spend a small amount of time before being heated or before reaching areas with smaller magnetic fields.

\subsection{Linear growth rates of the counter-streaming instability}

Another standard test is to study the linear phase of the counterstreaming instability. We use relativistic streaming velocities to validate the behavior of the algorithm for relativistic particle motions. Moreover, since magnetic fields are generated for this range of parameters, this test also probes the integration of $\boldsymbol{b}$.

The initial setup consists of two unmagnetized and cold counter-streaming electron-positron beams, with velocity $\pm \beta_{0} \hat{z}$ and associated Lorentz factor $\Gamma_{0}$. There is no background magnetic field, and the particles are loaded according to a drifting Maxwell-Boltzmann distribution. This situation is unstable, and the kinetic energy of the beam is converted into particle thermal kinetic energy and electromagnetic field energy, the initial perturbation coming from fluctuations due to the finite superparticle number. The linear instability spectrum is described by a branch comprising an electrostatic longitudinal two-stream mode and a transverse electromagnetic filamentation mode, the general case being an oblique mixed mode (Bret et al. 2010).

\subsubsection{Theoretical model}

We take the growth rates derived analytically by Michno \& Schlickeiser (2010) on the basis of a cold two-fluid model and denote this result as theoretical. Our thermal velocity $v_{\text {th }}$, identical for both species, is low enough to insure that thermal effects are negligible (Bret et al. 2010, Eq. (28)), but high enough to have a resolved Debye length and to avoid numerical instabilities (Appendix B). Our parameters are chosen such that the transverse filamentation mode always dominates. The fastest growing modes are those at large wavenumbers perpendicular to the beams, i.e., $k_{\perp} d_{\mathrm{e}} \gg \sqrt{2} / \Gamma_{0}^{3 / 2}$ (with $d_{\mathrm{e}}=c / \omega_{\mathrm{pe}}$ ), and that grow according to $b_{x}, b_{y} \propto \exp \left\{t / \tau_{\max }^{\text {theory }}\right\}$ with

$\tau_{\max }^{\text {theory }}=\frac{1}{2 \pi} \sqrt{\frac{\Gamma_{0}}{2}} \beta_{0}^{-1} T_{\mathrm{pe}}$.

The $k_{\perp}$-dependance of the growth rate is plotted in Fig. 3. We see that all modes above a few $d_{\mathrm{e}}^{-1}$ quickly reach the maximum growth rate $\tau_{\max }^{\text {theory }}=\tau^{\text {theory }}\left(k_{\perp}=+\infty\right)$.

\subsubsection{Method of measurement}

We measure the growth rates of the magnetic fields $b_{x}$ and $b_{y}$ with two methods. The first is a direct measure on the total energy curve, e.g., $\int \mathrm{d} V b_{x}^{2} \propto \exp (2 t / \tau)$ (see Fig. 4, top, for an illustration). It gives an effective growth rate that we denote by $\tau_{\text {tot en }}^{\mathrm{simu}}$, equal to $0.48 T_{\mathrm{pe}}$ in this case.

The second consists in following the time evolution of the Fourier modes of the fields. At a fixed time $t_{0}$, we compute the 2D Fourier transform of the fields in a plane $(x, z)$ with a fixed $y$, that we denote by $\mathrm{FT}_{y=y_{0}}\left(t_{0}, k_{x}, k_{z}\right)$. We then average the power spectrum over all the planes $y=$ cst to obtain the power spectrum $\mathrm{PS}\left(t_{0}, k_{x}, k_{z}\right)=\sum_{y_{0}}\left|\mathrm{FT}_{y=y_{0}}\left(t_{0}, k_{x}, k_{z}\right)\right|^{2}$. We then repeat this procedure for several $t_{0}$. The discrete mode spectrum is sampled with $\left(k_{z} d_{\mathrm{e}}, k_{x} d_{\mathrm{e}}\right)=2 \pi n_{x}\left(i / N_{z}, j / N_{x}\right)$, where $N_{z}$ and $N_{x}$ are the total number of cells in the $z$ and $x$ directions, and $i=0 \ldots N_{z} / 2$,

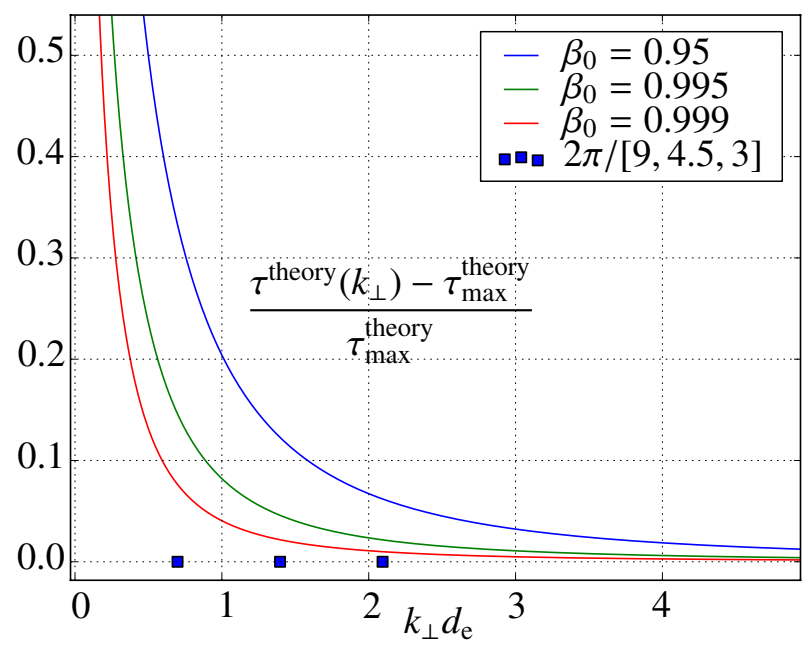

Fig. 3. Wavenumber dependance of the growth rate $\tau^{\text {theory }}\left(k_{\perp}\right)$, represented here as the deviation $\left[\tau^{\text {theory }}\left(k_{\perp}\right)-\tau_{\max }^{\text {theory }}\right] / \tau_{\max }^{\text {theory }}$. We recall that $\tau_{\max }^{\text {theory }}=\tau^{\text {theory }}\left(k_{\perp}=+\infty\right)$. From Eq. (69) of Michno \& Schlickeiser (2010). The squares are the resolution in $k_{\perp}$ for a box of transverse size $9,4.5$, and $3 d_{\mathrm{e}}$.

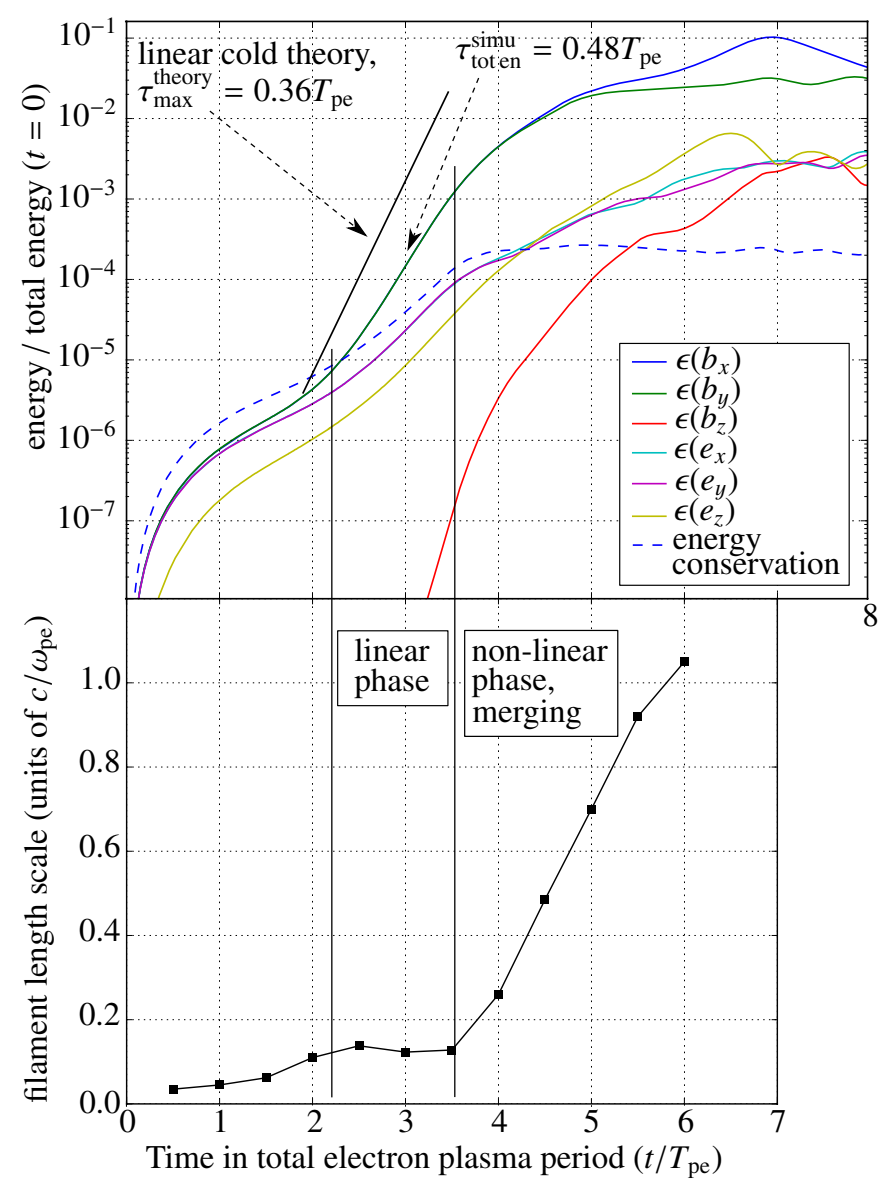

Fig. 4. Top: energy curves for the filamentation instability (case labeled Fig. 4 in Table 3). They are normalized by the total initial energy $E_{\text {tot }}^{0}$, which is mostly the kinetic energy of the particles. For example $\epsilon\left(b_{x}\right)=\int \mathrm{d} V B_{x}(t)^{2} /\left(2 \mu_{0}\right) / E_{\mathrm{tot}}^{0}$. The curve "energy conservation" is $\left(E_{\mathrm{tot}}^{0}-\right.$ total energy $\left.(t)\right) / E_{\mathrm{tot}}^{0}$. After $8 T_{\mathrm{pe}}$, the situation is more or less steady. Bottom: autocorrelation scale of the current amplitude.

$j=0 . . N_{x} / 2$. The spectral resolution in the direction perpendicular to the beam is thus $\Delta k_{\perp} d_{\mathrm{e}}=2 \pi n_{x} / N_{x}=2 \pi /$ (box width in $d_{\mathrm{e}}$ ). 
M. Melzani et al.: Apar-T: code, validation, and physical interpretation of particle-in-cell results

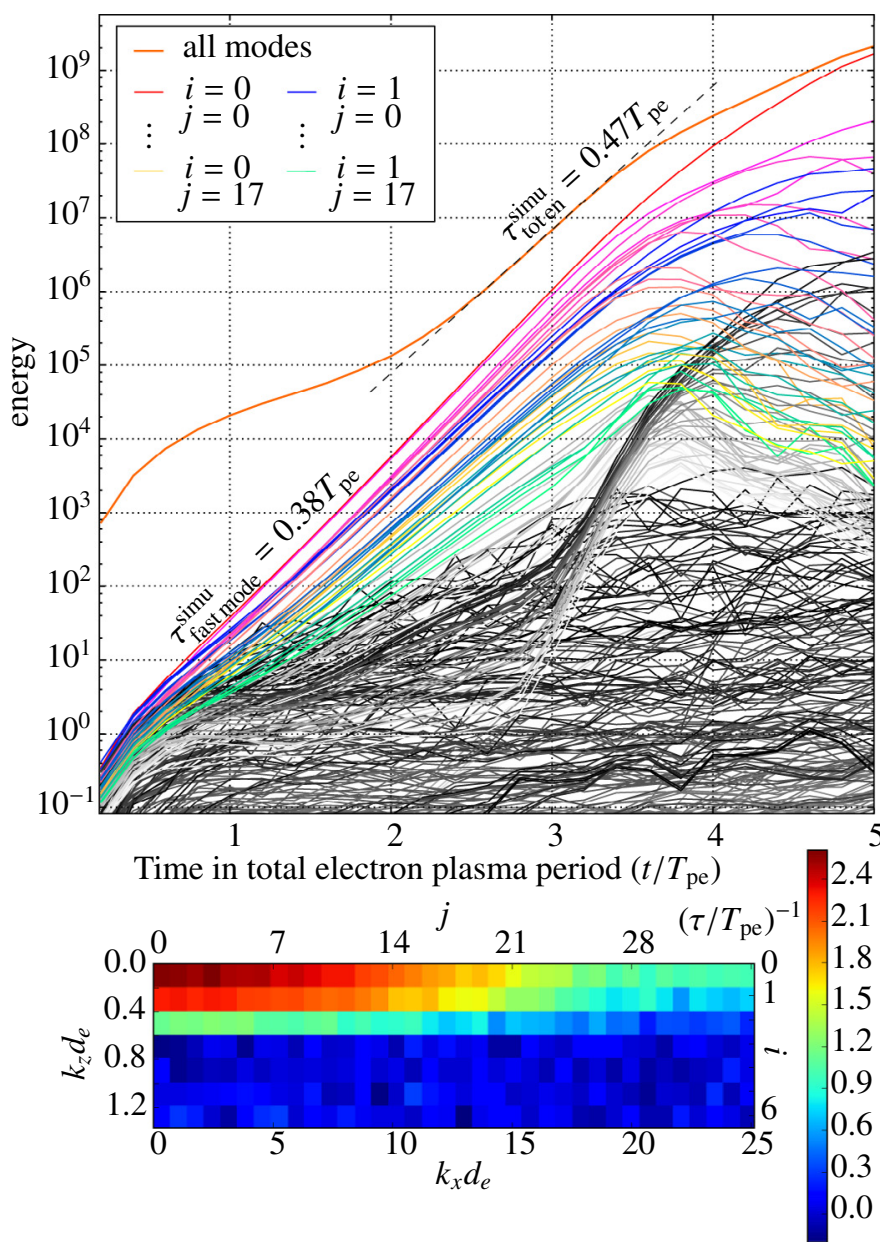

Fig. 5. Top: growth of individual Fourier modes for $b_{x}$ (for the run labeled Fig. 4 in Table 3). The modes shown are those for $i=0,1,2$, and $j=0,1, \ldots, 25$, and one mode every 100 modes for the remaining. We note that the graphic has been cut and that the weak modes actually fill a continuum down to an energy of $10^{-7}$. The sum of all $320 \times 90$ modes is shown in orange. We recall that mode $(i, j)$ corresponds to $\left(k_{z} d_{\mathrm{e}}, k_{x} d_{\mathrm{e}}\right)=2 \pi \times 20(i / 640, j / 180)$. Bottom: growth map of the Fourier modes, in units of $T_{\mathrm{pe}}$.

The squares in Fig. 3 represent this spectral resolution for the different box sizes that we use.

\subsubsection{Results}

Figure 5 is an example of the temporal evolution of the modes of $b_{x}$ for the same simulation as in Fig. 4. The sum of all modes grows at the same effective growth rate as the total energy in $b_{x}$ (to within $\pm 1 \%$ ), $\tau_{\text {tot en }}^{\text {simu }}=0.48 T_{\text {pe }}$. However, the fastest growing modes are those for $k_{z}=0$ and $0 \leq k_{x} d_{\mathrm{e}} \leq 5$, with $\tau_{\text {fast mode }}^{\text {simu }}=0.38 T_{\text {pe }}$, which is close to the cold-fluid result $\tau_{\max }^{\text {theory }}=0.36 T_{\text {pe }}$. It is seen from Fig. 5 that the large difference between the effective growth rate $\tau_{\text {tot en }}^{\text {simu }}$ and the growth rate of the fastest modes $\tau_{\text {fast mode }}^{\text {simu }}$ is due to a significant contribution of all the modes during the whole linear phase. The fastest mode thus never dominates the total energy in the linear phase. We suspect that this is due to the large noise level present in PIC simulations.

These results hold for all the test simulations that we conducted, which are summarized in Table 3. The effective growth rates $\tau_{\text {tot en }}^{\text {simu }}$ measured on the total energy present various levels of discrepancies with $\tau_{\max }^{\text {theory }}$, between $14 \%$ and $67 \%$. Figure 6

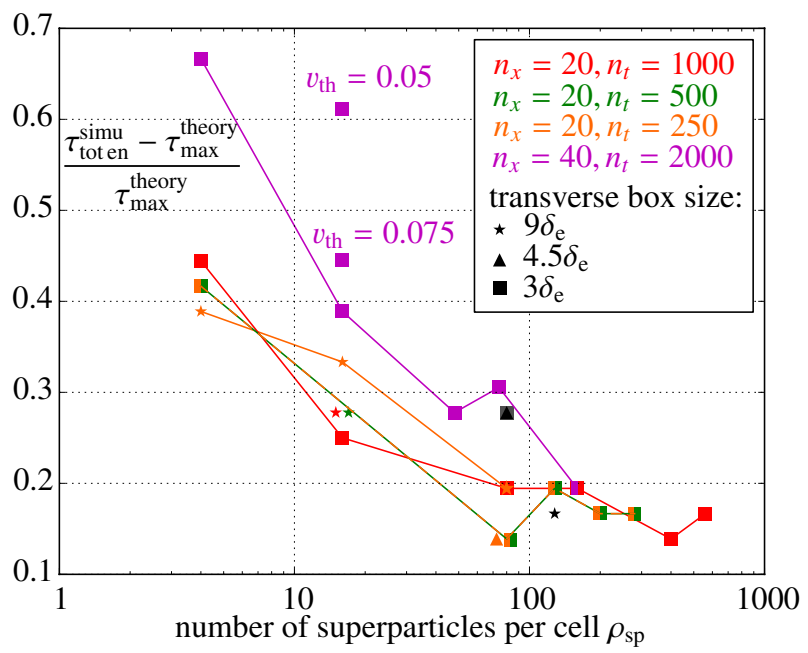

Fig. 6. Difference between the growth rate measured on the total energy curve $\tau_{\text {tot en }}^{\text {simu }}$ and the theoretical growth rate of the fastest modes $\tau_{\max }^{\text {theory }}$. Except when labeled otherwise, $v_{\text {th }}=0.1 c$. Each symbol corresponds to a transverse box size, and each color to fixed $\left(n_{x}, n_{t}\right)$.

shows the dependance of these discrepancies. There is a small sensitivity with respect to the timestep $\left(n_{t}\right)$ and the box size, and an important influence of the spatial resolution $\left(n_{x}\right)$. There is a systematic decrease in the difference when the superparticle number per cell $\rho_{\mathrm{sp}}$ is increased (all other parameters are kept constant). Since the fluctuation level in the PIC plasma decreases with increasing $\rho_{\mathrm{sp}}$, this indicates that the high fluctuation level excites all the modes and prevents the fastest ones from dominating the energy.

On the other hand, the growth rates $\tau_{\text {fast mode }}^{\text {simu }}$ measured on the fastest modes differ from $\tau_{\max }^{\text {theory }}$ by a more systematic factor, $14 \%$ for $\beta_{0}=0.95,7 \pm 1 \%$ for $\beta_{0}=0.995$, and $6 \%$ for $\beta_{0}=0.999$. These systematic differences can be explained by looking at the mode spectrum. In all the simulations, the fastest modes are for $k_{z}=0$ and $0 \leq k_{x} d_{\mathrm{e}} \leq 5-15$ (see, e.g., Fig. 5, bottom). Given the spectral resolution $\Delta k_{\perp} d_{\mathrm{e}}=2 \pi /\left(\right.$ box length in $\left.d_{\mathrm{e}}\right)$, these modes actually cover a portion of $k_{\perp} d_{\mathrm{e}}$ where the curves $\tau^{\text {theory }}\left(k_{\perp}\right)$ of Fig. 3 vary significantly and do not yet reach $\tau_{\max }^{\text {theory }}$. It explains the sign and order of magnitude of the difference $\tau_{\text {fast mode }}^{\text {simu }}-\tau_{\max }^{\text {theory }}$. It also explains the increasingly better agreement when $\beta_{0}$ increases.

We note that $\boldsymbol{e}$ and $b_{z}$ are zero in the linear two-fluid theory, and the fact that they are not zero in our simulation (see Fig. 4) reflects an early nonlinear evolution or the effects of fluctuations and correlations absent from the linear model but present in PIC simulations. These differences are discussed further in Sect. 5.

\subsection{Nonlinear evolution of the filamentation instability: filament merging}

We now consider the nonlinear phase of the filamentation instability. We study the same counter-streaming configuration as in Sect. 3.2, with the setup labeled by Fig. 4 in Table 3. The energy curves are shown in Fig. 4.

As a diagnostic, we focus on the filament growing and merging processes. The linear phase of the filamentation instability produces current filaments. Since they are threaded by parallel currents, they attract each other and, starting from the end of the linear phase, start to merge to produce larger and larger filaments (Medvedev et al. 2005). This is clearly visible in Fig. 7. 

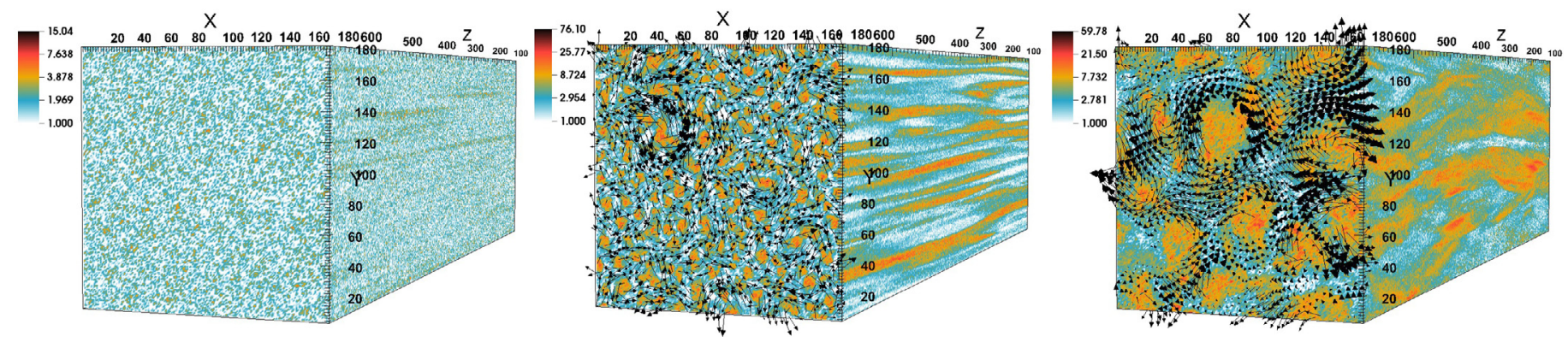

Fig. 7. Three snapshots of the filamentation instability, at times $2.5,4$, and $5.5 T_{\mathrm{pe}}$ (from left to right) for the simulation of Fig. 4 . Colors represent the current magnitude per cell (units are number of superparticles per cell times their mean velocity), while arrows are the magnetic field. Lengths are in cell number.

Table 3. Theoretical versus experimental values of the filamentation growth rate $\tau$.

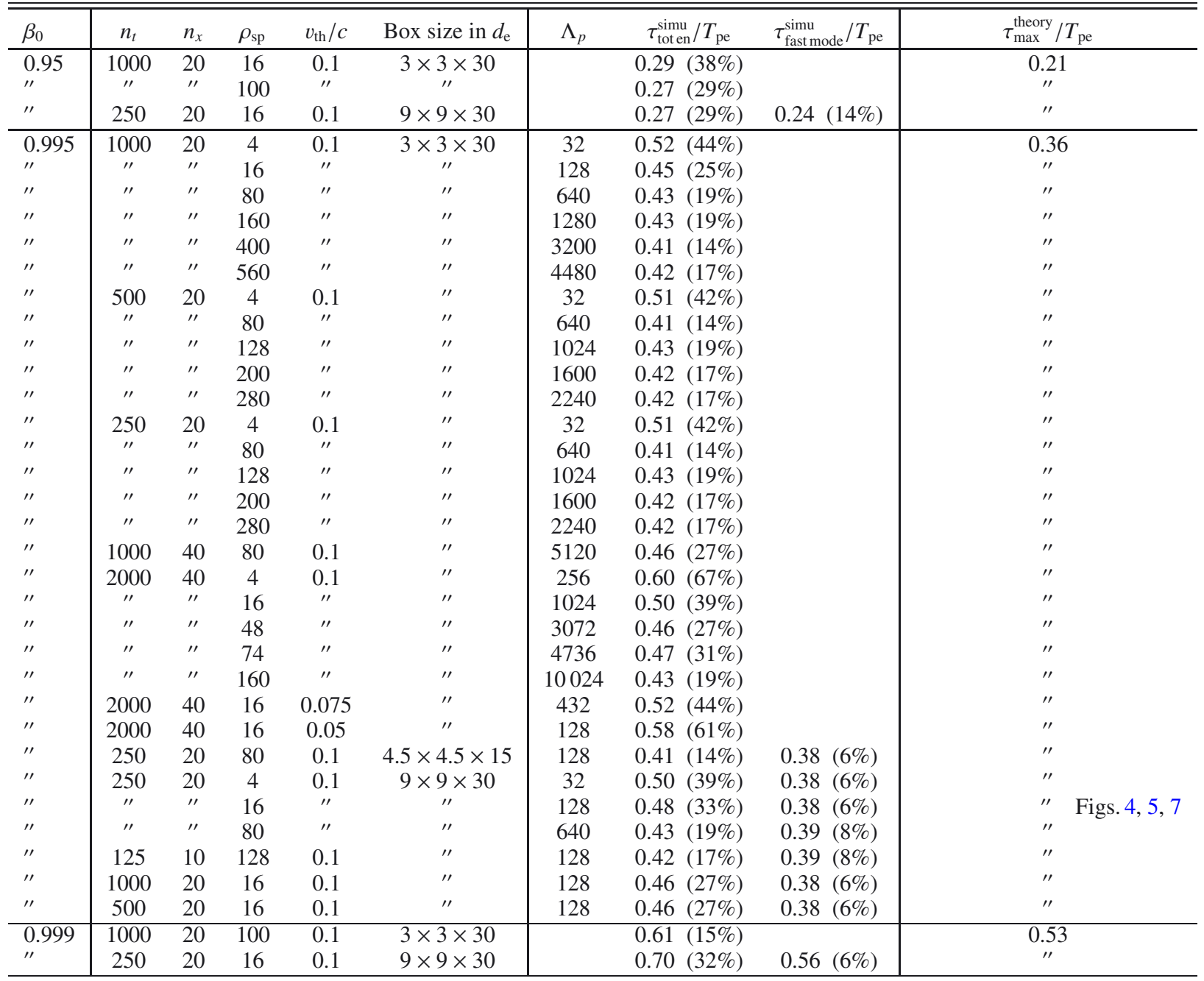

Notes. Numbers in parenthesis are the discrepancy with respect to $\tau_{\max }^{\text {theory }}$, i.e., $\left[\tau_{\text {tot en }}^{\text {simu }}-\tau_{\max }^{\text {theory }}\right] / \tau_{\max }^{\text {theory }}$ and $\left[\tau_{\text {fast mode }}^{\text {simu }}-\tau_{\max }^{\text {theory }}\right] / \tau_{\max }^{\text {theory }} . T_{\text {pe }}$ is the plasma period comprising all electrons, and $d_{\mathrm{e}}$ the plasma skin depth based on this period. We recall that $d_{\mathrm{e}}$ corresponds to $n_{x}$ cells. We also give the PIC plasma parameter $\Lambda_{p}=n_{\mathrm{e}, \mathrm{sp}} \lambda_{\mathrm{De}}^{3}=\rho_{\mathrm{sp}}\left(n_{x} v_{\mathrm{th}} / c\right)^{3}$ (see Sect. 5.1).

We measure the size of the filaments by computing the twodimensional autocorrelation function, in the $x-y$ plane, of the $z$-averaged current amplitude ( $z$ is the direction of the beams). This autocorrelation function is azimuthally averaged to obtain a radial function $\operatorname{corr}(r)$. We normalize $\operatorname{corr}(0)$ to 1 . The scale of the filaments is then taken to be five times the radius where $\operatorname{corr}(r)=0.8$ (taking this scale as the radius at which $\operatorname{corr}(r)$ first vanishes yields the same results).
The results are shown in Fig. 4 (bottom). We clearly see two regimes: one during the linear growth of the filamentation instability (from $t=2.2$ to $3.6 T_{\mathrm{pe}}$ ) where the filament correlation length is set by the wavelength of the fastest growing mode and remains constant, and one in the nonlinear regime (after $t=3.6 T_{\mathrm{pe}}$ ) where the filaments merge and thus quickly increase their size. In the second case, the growth is roughly linear with time, which agrees with the PIC simulation results of 
Table 4. Theoretical versus experimental values of the tearing growth rate $\tau$.

\begin{tabular}{l|ccccccc|ccc}
\hline \hline$\Theta$ & $\omega_{\text {ce }} / \omega_{\mathrm{pe}}$ & $\Gamma_{\mathrm{e}} U_{\mathrm{e}} / c$ & $L / d_{\mathrm{e}}$ & $n_{t}$ & $n_{x}$ & $\rho_{\mathrm{sp}}$ & Box size in $d_{\mathrm{e}}$ & $\tau^{\text {simu }} / T_{\mathrm{pe}}$ & $\tau^{\text {theory }} / T_{\mathrm{pe}}$ & $\left(\tau^{\text {simu }}-\tau^{\text {theory }}\right) / \tau^{\text {simu }}$ \\
\hline 0.01 & 0.2 & 0.14 & 0.72 & 250 & 15 & 1000 & $53 \times 0.3 \times 42$ & 5.4 & 5 & $8 \%$ \\
$\prime \prime$ & $\prime \prime$ & $\prime$ & $\prime \prime$ & $\prime \prime$ & $\prime \prime$ & $\prime \prime$ & $80 \times 0.3 \times 42$ & 5.4 & ${ }^{\prime \prime}$ & $8 \%$ \\
\hline 0.1 & 0.61 & 0.40 & 0.83 & 250 & 15 & 1000 & $53 \times 0.3 \times 42$ & 2.1 & 2 & $5 \%$ \\
\hline 1 & 1.82 & 0.68 & 1.62 & 250 & 15 & 1000 & $53 \times 0.3 \times 42$ & 2.5 & 2.7 & $7 \%$ \\
\hline
\end{tabular}

Notes. $T_{\mathrm{pe}}$ is the plasma period comprising all electrons, and $d_{\mathrm{e}}$ the plasma skin depth based on this period. We recall that $d_{\mathrm{e}}$ corresponds to $n_{x}$ cells. Other simulations were performed in the case $\Theta=0.01$, but with an initial setup slightly out of equilibrium, and they presented variations in the growth rates of less than $3 \%$ when $n_{x}, n_{t}$, and $\rho_{\mathrm{sp}}$ were doubled, or when $\rho_{\mathrm{sp}}$ was divided by two.

Dieckmann (2009) for a similar setup. After $t=6.5 T_{\text {pe }}$, the filament growth stops. However, we suspect that the periodic boundaries start influencing the dynamics at this point.

\subsection{Linear growth rates of the relativistic tearing instability}

We also study the linear phase of the tearing mode for a relativistic Harris sheet in a pair plasma. Contrary to the preceding case, this example provides a test of the algorithm in a situation where thermal effects are essential.

The equilibrium consists of a magnetic field

$\boldsymbol{B}=\hat{z} B_{0} \tanh \left(\frac{x}{L}\right)$,

sustained by a population of electrons and positrons of density $\propto 1 / \cosh ^{2}(x / L)$ flowing with opposite bulk velocities $U_{\mathrm{e}}=-U_{\mathrm{i}}$ in the $\pm y$ directions. We denote the associated Lorentz factor by $\Gamma_{\mathrm{e}}$, and the temperature of the two species by $\Theta=T /\left(m_{\mathrm{e}} c^{2}\right)$. The exact relations between the different parameters to satisfy the equilibrium are given in Appendix C. In particular, one should be careful to distinguish between quantities in the frame moving with one species (denoted with a prime) and quantities in the simulation frame. For example, contraction of the electron density leads to $\omega_{\mathrm{pe}}=\sqrt{\Gamma_{\mathrm{e}}} \omega_{\mathrm{pe}}^{\prime}$.

The superparticles are loaded according to a drifting Maxwell-Jüttner distribution with the method described in Sect. 4. There is no initial perturbation, and the instability grows out of the fluctuations produced by the finite number of superparticles.

As can be seen in Table 4, the bulk velocities and the temperatures of electrons and positrons are relativistic. Loading these distributions in a PIC code is a non-trivial task, and we have developed a special method for this. It is presented in Sect. 4.

The simulation domain is periodic along $z$ and $y$. Reflecting boundaries for particles and fields are present along the $x$ direction. There are no background particles, only that of the current sheet.

An example of the energy evolution is presented in Fig. 8. After some time, the system becomes unstable and the magnetic field starts reconnecting. As expected, $b_{z}$ dwindles while $b_{x}$ rises, which corresponds to the formation of magnetic islands. We measure the linear growth rate on $b_{x}$ as $\int \mathrm{d} V b_{x}^{2} \propto \exp (2 t / \tau)$. For comparison, we use the linear growth rates derived by Pétri \& Kirk (2007) by linearizing the Vlasov-Maxwell system around the drifting Maxwell-Jüttner distribution C.1 and the magnetic field of Eq. (5).

The results are summarized in Table 4. Discrepancies with Pétri \& Kirk (2007) range between 5\% and 8\%. These growth rates vary by less than $3 \%$ when the numerical resolution is doubled (i.e., when $\rho_{\mathrm{sp}}, n_{x}$, and $n_{t}$ are doubled all together). We restrict our analysis to total energy curves because contrary to the

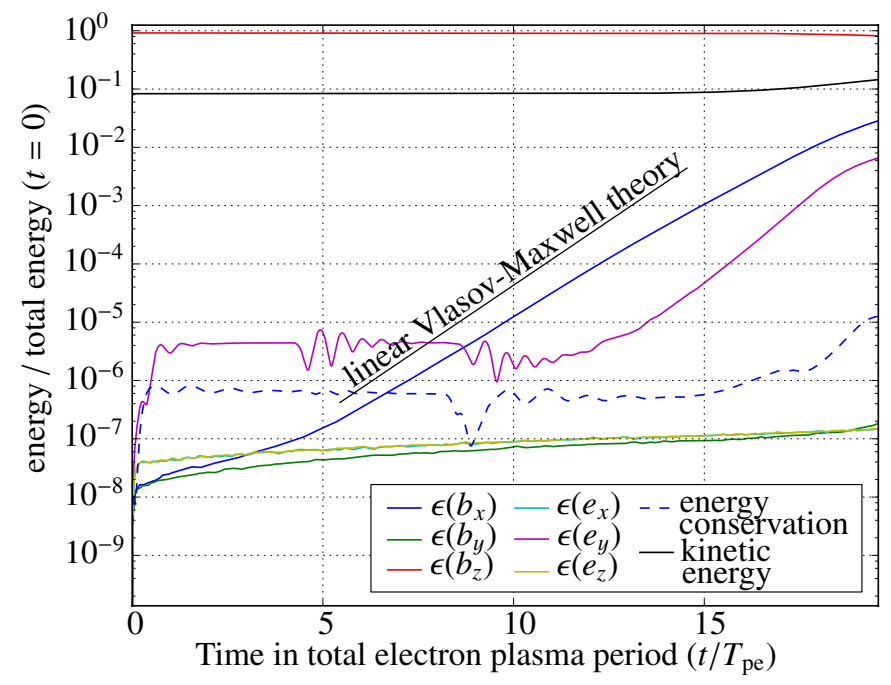

Fig. 8. Energy curves for the tearing instability (labeled Fig. 8 in Table 4). Shown are the energy curves, for example $\int \mathrm{d} V B_{x}(t)^{2} /\left(2 \mu_{0}\right)$. They are normalized by the total initial energy $E_{\text {tot }}^{0}$ (which is mostly the energy in $\left.b_{z}\right)$. The curve labeled "energy conservation" is $\left(E_{\mathrm{tot}}^{0}-\right.$ total energy $(t)) / E_{\mathrm{tot}}^{0}$.

case of the filamentation instability, the linear growth of the field energy spans several orders of magnitude and the fastest modes have enough time to dominate the total energy.

We note that when the simulation is launched, an electromagnetic wave is seen to propagate from the sheet in the $\pm x$ directions. This wave is a necessary consequence of the fact that at $t=0$ we load the superparticles by pairs of electron-positron at the same location, and we set a zero electric field everywhere (see Sect. 2.1). The system then has to relax from this very peculiar state: in less than one plasma period, charge screening is established and an electric field appears. It is this field that partly propagates outside of the sheet. It is then reflected on the $\pm x$ boundaries and propagates back to the sheet, causing the oscillations in $e_{y}$ seen in Fig. 8. We have checked that their incidence does not influence the linear growth by using different domain sizes.

It is more puzzling that the sheet contracts slightly just after the beginning of the simulation and the current magnitude at its center rises by $\$ 10 \%$. This may be related to the fact that our algorithm does not solve the Vlasov-Maxwell system in a strict sense (see also Sects. 5 and 6.3).

\section{Loading a relativistic particle distribution}

This section deals with the general problem of loading particles with momenta that reproduce a given distribution function. 
Very common cases are waterbag and Maxwell-Boltzmann distributions with a mean bulk velocity $U_{0}$. A simple method is then to load the particles in the frame comoving with the plasma, which is fairly easy because in this frame the distributions are isotropic, and then to add to every particle the velocity $U_{0}$ or, if $U_{0}$ is close to $c$, to boost every particle with the Lorentz boost corresponding to $U_{0}$. We will see, however, that this method is no longer correct when both $U_{0}$ and the rest frame distribution are relativistic, mainly because boosting particles in a PIC code does not boost space. We present here another method, correct in all cases ${ }^{2}$.

\subsection{Transformation of the distribution function}

We start by explaining how the particle distribution changes from one frame to another (see e.g. Mihalas \& Mihalas 1984; Pomraning 1973).

We consider a frame $\mathcal{R}$ where the plasma has a mean velocity $U_{0}$ and follows the distribution $f(\boldsymbol{x}, \boldsymbol{p})$. In the comoving frame or plasma rest frame $\mathcal{R}_{0}$, the plasma mean velocity is zero and follows the distribution $f_{0}(\boldsymbol{x}, \boldsymbol{p})$. We follow a group of particles. Seen from $\mathcal{R}$, they are in a volume $\mathrm{d}^{3} x$ around $x$ and have momentum $\boldsymbol{p}$ with a scatter $\mathrm{d}^{3} p$; seen from $\mathcal{R}_{0}$ these quantities change respectively to $\mathrm{d}^{3} x_{0}, \boldsymbol{x}_{0}, \boldsymbol{p}_{0}$, and $\mathrm{d}^{3} p_{0}$. The number of particles in our group is

$f_{0}\left(\boldsymbol{x}_{0}, \boldsymbol{p}_{0}\right) \mathrm{d}^{3} x_{0} \mathrm{~d}^{3} p_{0}=f(\boldsymbol{x}, \boldsymbol{p}) \mathrm{d}^{3} x \mathrm{~d}^{3} p$,

so that to find the link between $f$ and $f_{0}$ we have to find a relation between $\mathrm{d}^{3} x_{0} \mathrm{~d}^{3} p_{0}$ and $\mathrm{d}^{3} x \mathrm{~d}^{3} p$.

We start with the momentum. In the rest frame, our group of particles have momenta spanning a range $d^{3} p_{0}$. Seen in the boosted frame, their momenta transform according to the Lorentz transformation, and span a new range $\mathrm{d}^{3} p$. These two volumes are thus linked by the Jacobian of the Lorentz transformation, and it can be shown that

$\mathrm{d}^{3} p / \gamma=\mathrm{d}^{3} p_{0} / \gamma_{0}$

where $\gamma$ and $\gamma_{0}$ are the Lorentz factors associated to $\boldsymbol{p}$ and $\boldsymbol{p}_{0}$.

We now consider the space volumes. Because of space contraction/dilatation, the group of particles will occupy a different volume in different frames. We consider the frame $\mathcal{R}^{\prime}$ comoving with the group of particles. This is possible because the particles all move at nearly the same velocity $\boldsymbol{v}_{0}=\boldsymbol{p}_{0} / \gamma_{0}$. We denote by a prime all quantities seen from this frame. In $\mathcal{R}^{\prime}$, the particles occupy a volume $\mathrm{d}^{3} x^{\prime}$. Since only one direction is contracted, and since $\mathcal{R}^{\prime}$ moves relative to $\mathcal{R}_{0}$ with Lorentz factor $\gamma_{0}=\sqrt{1+p_{0}^{2}}$, we have the relation $\mathrm{d}^{3} x_{0}=\mathrm{d}^{3} x^{\prime} / \gamma_{0}$. Similarly, $\mathcal{R}^{\prime}$ moves relative to $\mathcal{R}$ with Lorentz factor $\gamma=\sqrt{1+p^{2}}$, and we have $\mathrm{d}^{3} x=\mathrm{d}^{3} x^{\prime} / \gamma$. All in all:

$\gamma \mathrm{d}^{3} x=\gamma_{0} \mathrm{~d}^{3} x_{0}$

From this, we deduce that

$f(\boldsymbol{x}, \boldsymbol{p})=f_{0}\left(\boldsymbol{x}_{0}, \boldsymbol{p}_{0}\right)$.

\footnotetext{
2 We note that after the submission of our article, Swisdak (2013) published a similar method.
}

\subsection{Why boosting particles from the rest frame is incorrect for relativistic distributions}

We now come back to PIC simulations. We assume that we load particles uniformly in space, with momenta following $f_{0}\left(\boldsymbol{x}_{0}, \boldsymbol{p}_{0}\right)$, and that we boost each particle with a velocity $U_{0}$. The momentum volume elements are then transformed according to Eq. (7), but positions are not changed. Equation (8) does not hold, and we obtain a particle distribution

$f_{\mathrm{PIC}}(\boldsymbol{x}, \boldsymbol{p})=f_{0}\left(\boldsymbol{x}, \boldsymbol{p}_{0}\right) \mathrm{d}^{3} p_{0} / \mathrm{d}^{3} p=f_{0}\left(\boldsymbol{x}, \boldsymbol{p}_{0}\right) \gamma_{0} / \gamma$.

The volume contraction/dilatation is not performed, and the factor $\gamma_{0} / \gamma$ does not cancel.

Consequently, boosting each particle from the rest frame leads to the expected distribution only if $\gamma_{0} / \gamma$ is independent of the particle. We can write this ratio as

$\frac{\gamma_{0}}{\gamma}=\frac{\gamma_{0}}{\Gamma_{0}\left(\gamma_{0}+p_{0, y} U_{0} / c\right)}$

with $p_{0, y}$ the $y$ component of $\boldsymbol{p}_{0}$ and $\Gamma_{0}=\left(1-U_{0}^{2} / c^{2}\right)^{-1 / 2}$, so that this is the case only if $p_{0, y} \ll 1$ (or if the boost is nonrelativistic, $\left.U_{0} \ll c\right)$. If $p_{0, y} \ll 1$, then $\gamma_{0} / \gamma \sim 1 / \Gamma_{0}$ and when it is inserted back into Eq. (10), we find the usual result of density contraction.

However, when the particle distribution is relativistic in the rest frame of the plasma, $\gamma_{0} / \gamma$ is not a constant factor and Eq. (10) does not have the expected dependence on momentum $\boldsymbol{p}$.

\subsection{Loading a drifting Maxwell-Jüttner distribution with arbitrary temperature and drift speed}

We now present a method for loading the superparticle momenta directly in the frame where the distribution has a bulk velocity.

In the literature, there is some agreement around the fact that the particle distribution of a relativistic plasma in thermodynamic equilibrium is given by the Maxwell-Jüttner distribution (Jüttner 1911; Cubero et al. 2007; Chacón-Acosta et al. 2010; Dunkel \& Hänggi 2009) and, even if some alternatives are also debated (Treumann et al. 2011), this is the distribution used in PIC simulations (e.g., Pétri \& Lyubarsky 2007; Zenitani \& Hoshino 2008; Jaroschek \& Hoshino 2009).

In the plasma rest frame, the Maxwell-Jüttner distribution is given by

$f_{0}\left(\boldsymbol{x}_{0}, \boldsymbol{p}_{0}\right)=n_{0} g_{0}\left(\boldsymbol{p}_{0}\right)=n_{0} \frac{\mu}{4 \pi K_{2}(\mu)} \exp \left\{-\mu \sqrt{1+p_{0}^{2}}\right\}$,

with $n_{0}$ the uniform particle number density and $g_{0}$ the momentum distribution, both in the restframe, $\mu=m c^{2} / T, \boldsymbol{p}=\gamma \boldsymbol{v} / c$, and $K_{2}$ the modified Bessel function of the second kind. If we now denote by $f$ the distribution in the frame where the plasma moves with a bulk velocity $c \beta_{0}$ (and associated Lorentz factor $\Gamma_{0}$ ), by $n$ the particle density, and by $g$ the momentum distribution, both in this same frame, we have $f(\boldsymbol{x}, \boldsymbol{p})=n g(\boldsymbol{p})$. Equation (9) then gives $g(\boldsymbol{p})=g_{0}\left(\boldsymbol{p}_{0}\right) \times n_{0} / n$. Now using $n=\Gamma_{0} n_{0}$, we arrive at

$g(\boldsymbol{p})=\frac{\mu}{4 \pi K_{2}(\mu) \Gamma_{0}} \exp \left\{-\mu \Gamma_{0}\left(\sqrt{1+p^{2}}-\beta_{0} p_{y}\right)\right\}$.

This distribution is normalized to unity: $\iiint \mathrm{d}^{3} \boldsymbol{p} g(\boldsymbol{p})=1$.

The main difficulty with Eq. (13) is that the variables $p_{x}, p_{y}$, and $p_{z}$ are coupled and cannot be chosen independently. The solution is to compute the marginal distribution for the variable $p_{y}$. 
M. Melzani et al.: Apar-T: code, validation, and physical interpretation of particle-in-cell results
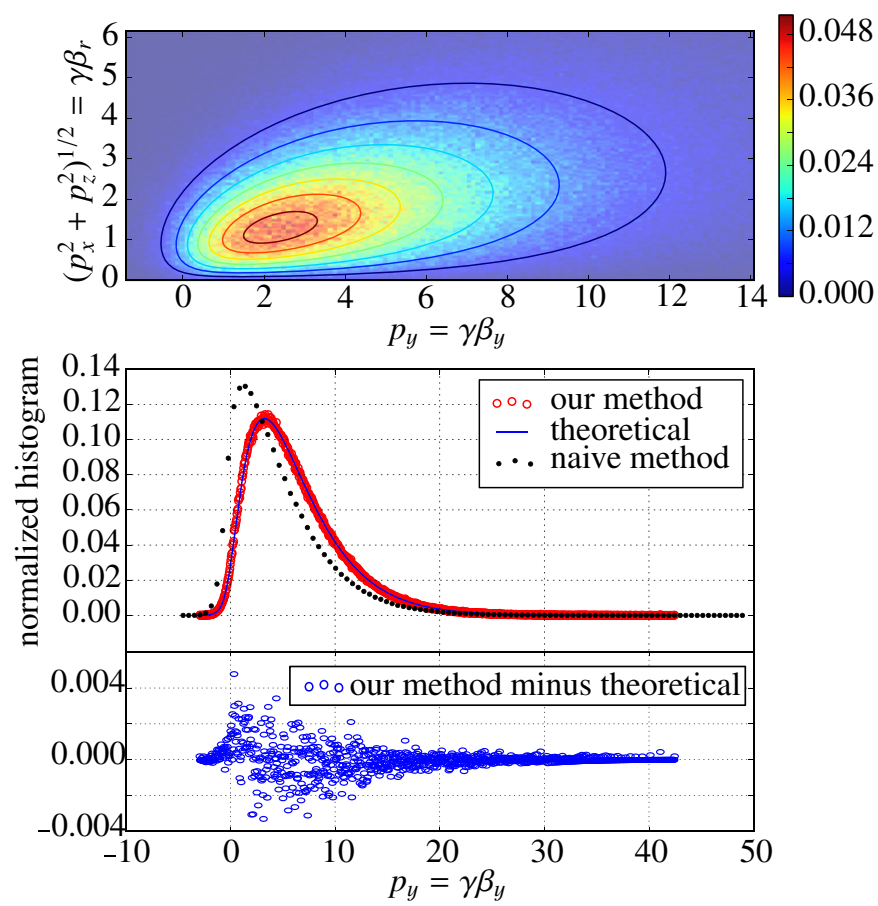

Fig. 9. Maxwell-Jüttner distribution for $T=m c^{2}$ and $\Gamma_{0} \beta_{0}=\sqrt{2}$. Upper plot: contours are drawn from the exact expression $2 \pi r g\left(r, 0, p_{y}\right)$, with $g$ from Eq. (13) and $\left(r, 0, p_{y}\right)$ defined in Appendix D. The background color map is the 2D histogram of $\left(\left(p_{x}^{2}+p_{z}^{2}\right)^{1 / 2}, p_{y}\right)$ for $10^{6}$ particles generated according to our method. Middle plot: normalized histogram of $p_{y}$ for the particles (red dotted), to be compared to the exact expression in Eq. (D.2) (blue line), and for comparison (black dots) the histogram of $p_{y}$ for particles generated the wrong way (initialization in the rest frame and boost of $\Gamma_{0}$ ). Bottom plot: difference between the red points and the blue curve.

With this, one can choose the $y$-component of $\boldsymbol{p}$ independently of the others, and then use the distribution $g\left(p_{\perp}, p_{y}\right)$ knowing $p_{y}$ to choose the component normal to $\boldsymbol{y}$. Details are presented in Appendix D, where we provide the expressions for the marginal distribution and the conditional probability, as well as a method for generating the velocity by using the cumulative distributions.

Figure 9 shows an example of the distribution generated with this method for $T=m c^{2}$ and $\Gamma_{0} \beta_{0}=1.41$, and compares it to the theoretical expectation and to the distribution obtained by boosting individually the superparticles from the restframe. We clearly see the accuracy of our algorithm, and the mismatch between the simpler boosting method and the expected result. We note that this mismatch can have significant consequences. For example, when used for the tearing instability of Sect. 3.4 it leads to large adjustments in the initial conditions, and for the most extreme temperatures to a complete disruption of the current sheet.

We also provide in Table 5 the analytical expressions of various quantities averaged over the Maxwell-Jüttner distribution, for example, the mean Lorentz factor, mean momentum, or the enthalpy. The method used to derive these expressions is presented in Appendix E. Besides being of general interest, these expressions served to further validate our generated distributions by comparing the analytical results of Table 5 with averages performed over particles generated with our method.

\section{Real, PIC, and Vlasov-Maxwell plasmas}

Particle-in-cell simulations have brought tremendous new insights into astrophysical plasmas, for example through studies of
Table 5. Useful averages for the Maxwell-Jüttner distribution $f(\boldsymbol{x}, \boldsymbol{p})=$ $n g(\boldsymbol{p})$ (Eq. (13)) of temperature $\Theta=1 / \mu=T /\left(m c^{2}\right)$.

\begin{tabular}{|c|c|c|c|}
\hline \multicolumn{4}{|c|}{ Without drift velocity: $U_{0}=0$} \\
\hline Parameter & Value & NR & UR \\
\hline$\langle\gamma\rangle$ & $\kappa_{32}(\mu)-\mu^{-1}$ & $1+3 \Theta / 2$ & $3 \Theta$ \\
\hline$\left\langle(\gamma \beta)^{2}\right\rangle$ & $3 \Theta \kappa_{32}(\mu)$ & $3 \Theta$ & $12 \Theta^{2}$ \\
\hline$\left\langle\beta^{2}\right\rangle$ & $3 \Theta \kappa_{12}(\mu)$ & $3 \Theta$ & $3 / 2$ \\
\hline$\left\langle\gamma \beta^{2}\right\rangle$ & $3 \Theta$ & & \\
\hline Larmor radius & $\left(c / \omega_{\mathrm{ce}}\right) \sqrt{2 \Theta \kappa_{32}(\mu)}$ & & \\
\hline Pressure $P$ & $n T$ & & \\
\hline Enthalpy $h$ & $\kappa_{32}(\mu)$ & $1+5 \Theta / 2$ & $4 \Theta$ \\
\hline $\begin{array}{l}\text { Adiabatic } \\
\text { exponent } \hat{\gamma}\end{array}$ & $1+\left(\mu \kappa_{32}(\mu)-\mu-1\right)^{-1}$ & $5 / 3$ & $4 / 3$ \\
\hline \multicolumn{4}{|c|}{ With a drift velocity $\boldsymbol{U}_{0}=U_{0} \hat{\boldsymbol{y}}$} \\
\hline Parameter & Value & NR & UR \\
\hline$\langle\boldsymbol{\langle v}\rangle$ & $\boldsymbol{U}_{0}$ & & \\
\hline$\langle\gamma \boldsymbol{v}\rangle$ & $\kappa_{32}(\mu) \Gamma_{0} \boldsymbol{U}_{0}$ & $\Gamma_{0} \boldsymbol{U}_{0}$ & $4 \Theta \Gamma_{0} \boldsymbol{U}_{0}$ \\
\hline$\langle\gamma\rangle$ & $\Gamma_{0} \kappa_{32}(\mu)-\frac{1}{4 \Gamma_{0}}$ & $\Gamma_{0}$ & $\frac{4 \Gamma_{0}}{u}-\frac{1}{u \Gamma_{0}}$ \\
\hline$\left\langle p_{x} v_{x}\right\rangle=\left\langle p_{z} v_{z}\right\rangle$ & $\Theta / \Gamma_{0}{ }^{\mu 1}$ & & \\
\hline$\left\langle p_{y} v_{y}\right\rangle$ & $\frac{\Theta}{\Gamma_{0}}+\Gamma_{0} \beta_{0}^{2} \kappa_{32}(\mu)$ & & \\
\hline$\left\langle\left(p_{i}-\left\langle p_{i}\right\rangle\right)\left(v_{j}-\right.\right.$ & $\delta_{i j} \Theta / \Gamma_{0}$ & & \\
\hline
\end{tabular}

Notes. We define $\kappa_{i j}(\mu)=K_{i}(\mu) / K_{j}(\mu)$, with $K_{n}$ the modified Bessel function of the nth kind. The Larmor radius is defined by $\left\langle r_{\mathrm{ce}}\right\rangle=$ $\left\langle\left(\gamma v_{\perp}\right)^{2}\right\rangle^{1 / 2} / \omega_{\text {ce }}$, the pressure by $P=(1 / 3) n\langle\boldsymbol{v} \cdot(\gamma m \boldsymbol{v})\rangle$, the enthalpy by $h=\left(n\left\langle\gamma m c^{2}\right\rangle+P\right) /\left(n m c^{2}\right)$, and the adiabatic exponent by $\hat{\gamma}-1=$ $P /\left(n\langle\gamma-1\rangle m c^{2}\right)$. NR means non-relativistic limit $\left(\Theta \rightarrow 0, \kappa_{32}(\mu) \sim\right.$ $1+5 \Theta / 2)$, and UR ultra-relativistic limit $\left(\Theta \rightarrow+\infty, \kappa_{32}(\mu) \sim 4 \Theta\right)$, with in both cases no constraints on $\Gamma_{0}$.

kinetic instabilities in their nonlinear phase, kinetic turbulence, particle acceleration via the Fermi-process, or 3D magnetic reconnection and the associated particle acceleration. However, as we will detail in this section, there remain a number of questions with respect to the degree to which PIC models are able to completely mirror real plasmas.

The modeling of a real plasma by a PIC plasma implies two steps (see the right branch of Fig. 12): the grouping of many real particles into a single superparticle, known as coarse-graining, and the discretization of the equations with the presence of a grid. Each of these steps raises questions:

1. Coarse-graining: Is plasma behavior still expected with so few superparticles per Debye sphere? Is the noise level too large? With this, do nonlinearities appear sooner than in real plasmas? Does the PIC plasma remain collisionless? And what are we losing when we gather the particles into the superparticles?

2. Discretization and grid: At least for explicit schemes, they bring with them numerical stability problems, reviewed in Appendix B. Moreover, the interpolation of superparticle quantities to grid points implies a finite volume for the superparticles, which in turn implies a vanishing two-point force at short distances and thus reduces drastically the influence of collisions; it helps the PIC plasma to be collisionless, but is it enough? And what are the consequences of having superparticles whose sizes reach a significant fraction of the Debye length?

We discuss some of these questions in Sects. 5.1 to 5.3.

The distinguishing feature of the Vlasov-Maxwell description of a plasma is the absence of collisions and of correlations between particles. Given the two preceding points, we can wonder if a PIC plasma can be described by the Vlasov-Maxwell 
system, or if it has too few superparticles per cell and thus correlation levels that are too high for this description to be accurate. The differences between PIC and Vlasov-Maxwell descriptions are examined in Sect. 5.4.

\subsection{The plasma parameter $\Lambda$ : a coarse-graining dependent quantity}

As said earlier and expressed in Eq. (1), a real plasma is represented in the computer by grouping many particles into superparticles. This is what is called coarse-graining. Unlike fluid equations $^{3}$, Eqs. (2a)-(2e) are not invariant under coarse-graining (because of the definition of the current, Eq. (2e)). The prototype of $p$-dependent quantities is the plasma parameter ${ }^{4} \Lambda=n \lambda_{\mathrm{D}}^{3}$, which is close to the number of particles per Debye sphere (we recall that $p$ is the number of particles per superparticles, $\lambda_{\mathrm{D}}$ the Debye length and $n$ the real particle number density).

The plasma parameter $\Lambda$ also expresses the ratio of the particles' kinetic energy to their electrostatic potential energy of interaction and, as such, varies as $1 / p$ because kinetic energy is proportional to the superparticles' mass $m_{\mathrm{sp}} \propto p$ while charge interaction energy involves their charge $q_{\mathrm{sp}}^{2} \propto p^{2}$. This can be seen directly by writing $\Lambda_{p}=n_{\mathrm{sp}} \lambda_{\mathrm{D}}^{3}$ for the superparticle plasma, with $n_{\mathrm{sp}}$ the number density of superparticles. The Debye length, being derived from fluid theory, is invariant under coarse-graining, and since $n=p \times n_{\text {sp }}$, one has that

$\Lambda_{p}=\frac{\Lambda}{p}$

with $\Lambda=\Lambda_{p=1}$ the real plasma parameter.

In a real plasma $\Lambda$ ranges from $10^{4}$ to $10^{20}$ (for example $\Lambda \sim 10^{6}$ in solar coronal loops; $10^{12}$ in the magnetotail, magnetopause, or in typical Crab flares; $10^{17}$ in AGN jets), while in computer experiments where we have to simulate thousands to millions of Debye spheres, $\Lambda$ reaches hardly a few tens (for a discussion see, e.g., Bykov \& Treumann 2011, Sect. 4). The corresponding number of particles per superparticles then reaches $p \sim 10^{3}$ to $10^{19}$. The question of the relevance of PIC simulations for describing collisionless plasmas has thus been asked from the beginning, and concerns both terms, collisionless and plasma, that we now discuss.

\subsubsection{Plasma behavior}

A weakly coupled plasma is characterized by the predominance of collective effects over individual effects. The ratio of these effects is contained in the plasma parameter $\Lambda_{p}$, which can be seen as the ratio of collective behavior (the interaction of one particle with the electromagnetic fields collectively generated by all others, which is coarse-graining independent) to binary effects (which are proportional to $q_{\mathrm{sp}}^{2} / m_{\mathrm{sp}} \propto p$ ).

Since plasma behavior, with Debye screening and local charge neutrality, requires a high plasma parameter, it is wise to ask how large it should be in a PIC plasma. Birsdall \& Langdon (1985, chap. 1) and Hockney \& Eastwood (1988) have shown

\footnotetext{
3 By a fluid model we mean any set of equations where the individual nature of the particles has been smoothed. This is the case of the MHD family, two-fluid models, or the Vlasov-Maxwell system.

4 In a fully ionized plasma, all coarse-graining dependent quantities can be expressed as the product of a fluid quantity (which is coarsegraining independent) and a parameter expressing a number of particles per fluid volume. Examples of these parameters include $\Lambda=n \lambda_{\mathrm{D}}^{3}$, $n\left(c / \omega_{\mathrm{pe}}\right)^{3}, n\left(c / \omega_{\mathrm{pi}}\right)^{3}, \ldots$
}

that it is not necessary for this ratio to be as high as in real plasmas, and that a $\Lambda_{p}$ of about a few suffices for correct plasma behavior.

\subsubsection{Collisionless behavior}

A plasma behaves collisionlessly if the time and length scales of interest are negligible toward the collision time and the meanfree path, respectively. Since the collision time scales as $\Lambda_{p}$ times the plasma period, it is not clear whether a PIC computer plasma with a plasma parameter on the order of unity will be collisionless.

Particle-in-cell plasmas are helped by the superparticle finite sizes, which imply that the two-point force decreases to zero for separations smaller than this size. This fact, albeit degrading the accuracy of single particle dynamics, greatly reduces the relative importance of binary collisions so that in order to correctly simulate a collisionless plasma for scales accessible in simulations, one has to insure that (Birsdall \& Langdon 1985; Hockney \& Eastwood 1988, chap. 1)

$\Lambda_{p}=n_{\mathrm{sp}} \lambda_{\mathrm{D}}^{3}=\rho_{\mathrm{sp}} \tilde{\lambda}_{\mathrm{D}}^{3}>$ a few.

(Here, $\tilde{\lambda}_{\mathrm{D}}=\lambda_{\mathrm{D}} / X_{0}$ is the normalized Debye length. It can be expressed as $\tilde{\lambda}_{\mathrm{D}}=n_{x} v_{\text {th }} / c$ with $v_{\text {th }}=\sqrt{T / m}$.)

This is all the more true if $r_{\mathrm{c}}<X_{0}$, where $X_{0}$ is the grid size and $r_{\mathrm{c}}$ is the effective collision radius for Coulomb encounters, expressed by equating the kinetic energy of the meeting particles to their potential energy of interaction: $r_{\mathrm{c}}=$ $q_{\mathrm{sp}}^{2} /\left(4 \pi \epsilon_{0} T\right)=\lambda_{\mathrm{D}} / \Lambda_{p}$ ( $r_{\mathrm{c}}$ is also $p$-dependent $)$. The Debye length must be resolved for reasons of numerical stability (Sect. 3.1 and Appendix B), so that we arrive at an optimal ordering $r_{\mathrm{c}}<X_{0}<\lambda_{\mathrm{D}}$, which is allowed only if, again, $\lambda_{\mathrm{D}} / r_{\mathrm{c}}=$ $\Lambda_{p}>1$.

However, because the PIC model is a description of a plasma of cloud charges, grazing collisions will still be present and will lead to thermalization (Birsdall \& Langdon 1985, Chap. 1; Hockney \& Eastwood 1988, Chaps. 1 and 9), a point discussed in the next section.

\subsection{Thermalization time: a coarse-graining dependent quantity}

In a PIC plasma, the behavior of plasma quantities depending on $\Lambda$ can be guessed by replacing $\Lambda$ by $\Lambda_{p}$. This is the case for the thermalization time of a plasma by grazing Coulomb collisions (Spitzer 1965) or by electric field fluctuations (Birsdall \& Langdon 1985, p. 282), which is on the order of $t_{\text {th }} \sim T_{\mathrm{P}} \times \Lambda$ (with $T_{\mathrm{P}}$ the plasma period). This has two important consequences:

- We expect $t_{\text {th }}$ to depend on resolution and coarse-graining, roughly as

$$
\frac{t_{\mathrm{th}}}{T_{\mathrm{P}}} \propto \Lambda_{p}=\rho_{\mathrm{sp}} \tilde{\lambda}_{\mathrm{D}}^{3}
$$

- Since $\Lambda_{p}=\Lambda / p$ is several orders of magnitude smaller than the real plasma parameter $\Lambda$, we expect the thermalization by grazing collisions and fluctuations to be vastly more efficient in PIC codes than in reality.

This can have important consequences in simulations where thermalization plays a key role. For example in real collisionless shocks, the mean free path for collisions $l_{\text {mean free path }}$ is far larger than the shock thickness $\Delta_{\text {shock }}$ and the thermalization processes are collisionless kinetic instabilities. Since the mean free 
M. Melzani et al.: Apar-T: code, validation, and physical interpretation of particle-in-cell results
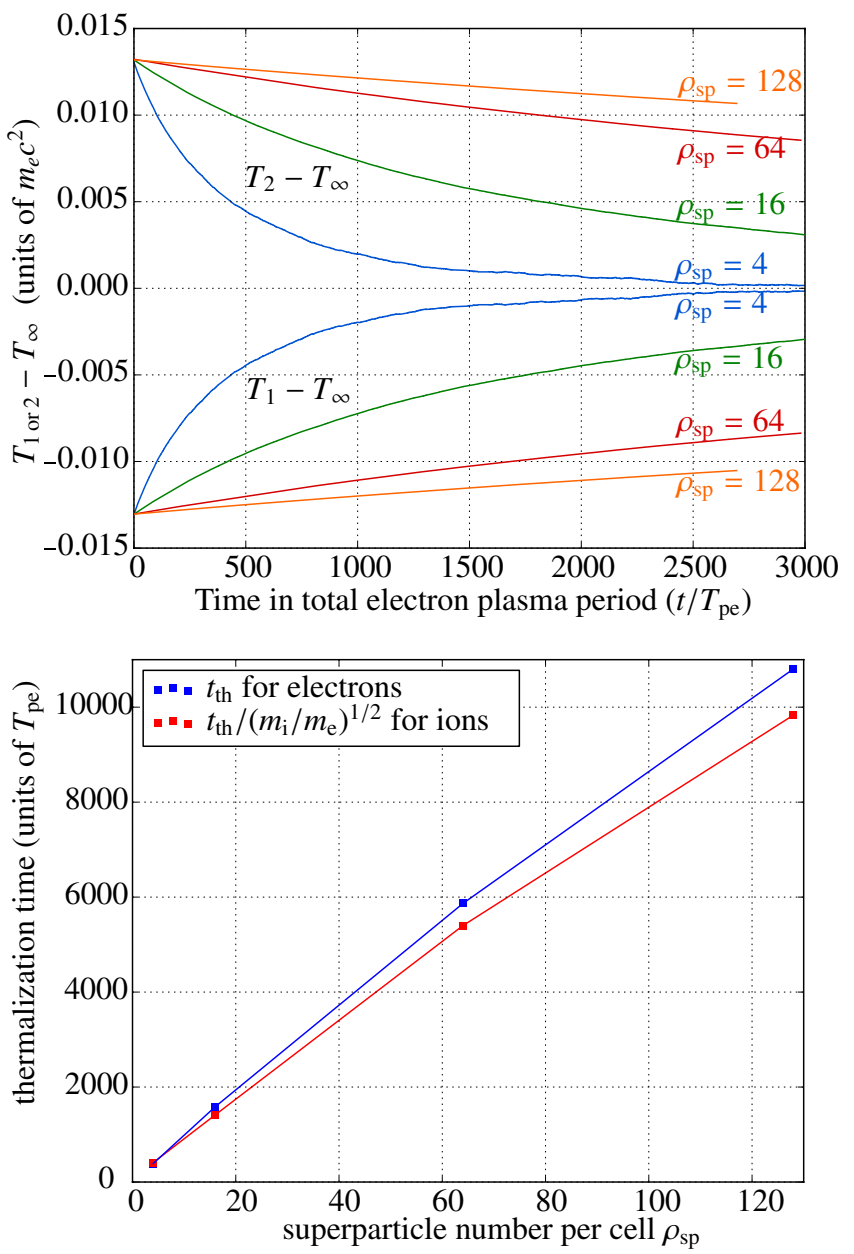

Fig. 10. Top: electron temperatures for the hot (2) and cold (1) plasmas, from four simulations with different $\rho_{\text {sp }}$. The curves for ion temperatures are similar, except for an overall time dilatation by a factor $\sim\left(m_{\mathrm{i}} / m_{\mathrm{e}}\right)^{1 / 2}=5$. In this figure we use $T_{\infty}(t)=\left(T_{1}(t)+T_{2}(t)\right) / 2$. Except for $\rho_{\text {sp }}=4$ where there is significant numerical heating, $T_{\infty}(t)$ is constant in time. Bottom: half-thermalization time for ions and electrons, versus number of superparticles per cell. For ions, we have plotted $t_{\mathrm{th}} /\left(m_{\mathrm{i}} / m_{\mathrm{e}}\right)^{1 / 2}$. The times reported are measured as the initial slope of the temperature curves in a log-lin plot, and thus correspond to $t_{\mathrm{th}} / 2$. We see the scaling $t_{\mathrm{th}} \propto \rho_{\mathrm{sp}}$.

path $l_{\text {mean free path }}^{\mathrm{PIC}} \propto \Lambda_{p}$ in a PIC plasma is smaller by a factor of $p \sim 10^{10}$ than in a real plasma, it is not obvious that the ordering $l_{\text {mean free path }}^{\text {PIC }} \gg \Delta_{\text {shock }}$ still holds. To truly describe a collisionless shock with a PIC algorithm, one has to be careful that the unphysically fast thermalization by collisions or fluctuations remains slower than thermalization by kinetic instabilities.

To illustrate the dependance of the collision and fluctuation induced thermalization time, we present simulations that initially have two thermal ion-electron plasmas. The first is cold, with a temperature $T_{1, \mathrm{e}}(0)=T_{1, \mathrm{i}}(0)=1.6 \times 10^{-3} m_{\mathrm{e}} c^{2}$ for its electrons and ions, while the second is hot, with $T_{2, \mathrm{e}}(0)=$ $T_{2, \mathrm{i}}(0)=1.8 \times 10^{-2} m_{\mathrm{e}} c^{2}$. The mass ratio is $m_{\mathrm{i}} / m_{\mathrm{e}}=25$. The four species interact via collisions and correlations (no sign of plasma kinetic instabilities were found) and tend to reach the same final temperature

$T_{\infty}=\frac{T_{1, \mathrm{e}}(0)+T_{2, \mathrm{e}}(0)}{2}$.

Particle-in-cell results are shown in Fig. 10 (top) for the electrons, for four simulations with a number of superparticles per cell (including all species) $\rho_{\mathrm{sp}}=4,16,64$, or 128 . The other parameters are kept fixed: $n_{x}=25, n_{t}=500$, and box size of $25^{3}$ cells. It results in $\Lambda_{\infty}=0.25 \rho_{\mathrm{sp}}\left(n_{x} \sqrt{T_{\infty} / m_{\mathrm{e}} c^{2}}\right)^{3}=15$, 61,243 , or 485 . The temperatures are measured with $T=$ $m \sum_{\mathrm{sp}} \boldsymbol{v}_{\mathrm{sp}}^{2} / 3$, where the sum runs over all the superparticles of a given species. In Fig. 10 (top) we clearly see a slower thermalization as $\rho_{\text {sp }}$ increases.

To evaluate the thermalization times, we use the result of Spitzer (1965): for two species at temperature $T_{1}$ and $T_{2}$, thermalization occurs according to

$$
\begin{aligned}
\frac{\mathrm{d} T_{1}}{\mathrm{~d} t} & =-\frac{\mathrm{d} T_{2}}{\mathrm{~d} t}=\frac{T_{2}-T_{1}}{t_{\mathrm{th}}}, \\
t_{\mathrm{th}} & =\frac{3 \pi}{2 \sqrt{2 \pi}} \frac{n \omega_{\mathrm{p} 1}^{-2} \omega_{\mathrm{p} 2}^{-2}}{\ln \Lambda^{\mathrm{c}}}\left(\frac{T_{1}}{m_{1}}+\frac{T_{2}}{m_{2}}\right)^{3 / 2},
\end{aligned}
$$

with $n=n_{1}=n_{2}$ the particle number density, $\omega_{\mathrm{p} i}=$ $\sqrt{n e^{2} /\left(\epsilon_{0} m_{i}\right)}$, and $\ln \Lambda^{\mathrm{c}}$ the Coulomb logarithm, or the logarithm of the ratio of the largest to closest distances used in the collision integral. In a PIC code, $\ln \Lambda^{\mathrm{c}}=\ln \lambda_{\mathrm{D}} / X_{0}$. Clearly, $\left(T_{1}(t)+T_{2}(t)\right) / 2$ is constant and equal to $T_{\infty}$. It follows that if mass $m_{1}$ and $m_{2}$ are equal, the thermalization time is also constant and can be written

$t_{\mathrm{th}}=\frac{3}{2 \sqrt{\pi}} \frac{2 \pi}{\omega_{\mathrm{p} 1}} \frac{\Lambda_{\infty}}{\ln \Lambda^{\mathrm{c}}}$,

with $\Lambda_{\infty}=n\left[\epsilon_{0} T_{\infty} /\left(n e^{2}\right)\right]^{3 / 2}$ the plasma parameter based on the temperature $T_{\infty}$. It also follows that the temperatures vary exponentially as $T_{1}=T_{\infty}-0.5\left[T_{2}(0)-T_{1}(0)\right] \exp \left\{-2 t / t_{\text {th }}\right\}$, and similarly for $T_{2}$.

Here we do not find the temperature curves to be strictly exponential, mainly because there are four species. The cold electrons interact with the hot electrons on a timescale $t_{0}$, but also with the hot ions on a timescale $m_{\mathrm{i}} / m_{\mathrm{e}} t_{0}=25 t_{0}$. The cold ions are heated by interactions with the hot ions on a timescale $\left(m_{\mathrm{i}} / m_{\mathrm{e}}\right)^{1 / 2} t_{0}=5 t_{0}$, and by interactions with the hot electrons on a timescale $m_{\mathrm{i}} / m_{\mathrm{e}} t_{0}=25 t_{0}$. Since the cold ions are heated more slowly than the cold electrons, a temperature difference between these two components appears and they also start heating or cooling each other. Nevertheless, given the separation of scales we expect a measure of the slope around $t=0$ to reflect the electron-electron or ion-electron thermalization times when measured on the electron or ion temperature curves, respectively.

Results are shown in Fig. 10 (bottom). We see that the relation $t_{\text {th }} \propto \rho_{\text {sp }}$ is roughly correct for both electrons and ions. We also underline the difference with a real plasma, where $t_{\mathrm{th}} / T_{\mathrm{pe}} \sim \Lambda$ reaches $10^{10}$ or more, while it is on the order of $\Lambda_{p} \leq 10^{4}$ in PIC simulations.

\subsection{Field fluctuation level dependence: coarse-graining and finite superparticle size}

The previous section showed that the behavior of PIC plasma quantities can be guessed by the substitution $\Lambda \rightarrow \Lambda_{p}$. While it is true for orders of magnitude estimates, this recipe is, however, not exact, and coarse-graining dependent quantities generally follow other relations than their real counterparts with respect to physical parameters (temperature, Debye length, plasma parameter, etc.). The main reason for this is that the finite volume of the superparticles implies a cutoff of the physical processes at smaller scales, an effect that becomes even more important when the superparticle size is close to the Debye length: $\lambda_{\mathrm{D}} / X_{0}=\tilde{\lambda}_{\mathrm{D}}$ typically ranges between one (or less) and ten in simulations. 
This section illustrates this double dependence $\left(\Lambda \rightarrow \Lambda_{p}\right.$ and superparticle size) with a detailed study of the level of electric field fluctuations $\varepsilon$ in a PIC thermal plasma.

In a real plasma in thermal equilibrium, it is given by (Callen 2006, Sect. 1.1)

$\varepsilon=\frac{\left\langle\epsilon_{0} \boldsymbol{E}^{2} / 2\right\rangle}{3 n T / 2} \sim \frac{1}{\Lambda}$

where the symbol $\langle\cdot\rangle$ denotes an average over space.

Dieckmann et al. (2004) studied the spectrum of thermal fluctuations in a PIC plasma, but without investigating their levels. Hockney (1971, see also Hockney \& Eastwood 1988) measured ratios like $\varepsilon$ in a series of 2D simulations of thermal plasmas, and found a good agreement with the empirical formula $\varepsilon \propto\left(\tilde{W}^{2}+\rho_{\mathrm{sp}} \tilde{\lambda}_{\mathrm{D}}^{2}\right)^{-1}$, where $\tilde{W}$ is the superparticle geometrical size in number of cells. Its algorithm was two dimensional, electrostatic, and based on the integration of the Poisson equation.

We perform these simulations with our 3D electromagnetic code and measure the level of energy in the electric field. We use thermal velocities from $0.04 c$ to $0.10 c, \rho_{\mathrm{sp}}$ from 2 to 500 , and $n_{x}$ from 10 to 128 . It results in $\tilde{\lambda}_{\mathrm{D}}=\lambda_{\mathrm{D}} / X_{0}$ from 0.4 to 12.8 , and in $\Lambda_{p}=n_{\mathrm{sp}} \lambda_{\mathrm{D}}^{3}=\rho_{\mathrm{sp}} \tilde{\lambda}_{\mathrm{D}}^{3}$ from 0.1 to 75000 . The fluctuation levels do not depend on the timestep (which varies from $n_{t}=2000$ down to close to the Courant limit $\Delta t \sim X_{0} / c$ ) nor on box size (which is always bigger than $n_{x}$ ). We use a pair plasma, but increasing the mass of the ions would only multiply the fluctuation levels by a constant factor.

The results are summarized in Fig. 11: $\varepsilon$ is found to be proportional to $\left(\tilde{\lambda}_{\mathrm{D}}-\arctan \tilde{\lambda}_{\mathrm{D}}\right) / \rho_{\mathrm{sp}} \tilde{\lambda}_{\mathrm{D}}^{3}$, and not exactly to $1 / \Lambda_{p}$. To explain this, we generalize the computation of Hockney to three dimensions.

For a plasma in thermal equilibrium, the energy in the electric field at location $(\boldsymbol{x}, t)$ can be evaluated by adding the electric field produced at $\boldsymbol{x}$ by charges at location $\boldsymbol{x}_{0}$ and having a velocity $\boldsymbol{v}_{0}, \boldsymbol{E}_{\boldsymbol{x}_{0}, v_{0}}(\boldsymbol{x}, t)$

$\frac{\epsilon_{0}\left\langle\boldsymbol{E}^{2}(\boldsymbol{x}, t)\right\rangle}{2}=\int \mathrm{d}^{3} \boldsymbol{v}_{0} \mathrm{~d}^{3} \boldsymbol{x}_{0} \frac{\epsilon_{0} \boldsymbol{E}_{\boldsymbol{x}_{0}, v_{0}}^{2}(\boldsymbol{x}, t)}{2} f_{0}\left(\boldsymbol{x}_{0}, \boldsymbol{v}_{0}\right)$,

where $\langle\cdot\rangle$ means an ensemble average (which coincides with a spatial average); $\boldsymbol{E}_{\boldsymbol{x}_{0}, \boldsymbol{v}_{0}}(\boldsymbol{x}, t)$ is a generalization of the Debye electric field for moving particles (Nicholson 1983, Chap. 9); and Eq. (21) can be evaluated for a plasma of finite-sized particles as (Hockney 1971; Birsdall \& Langdon 1985)

$\frac{\left\langle\epsilon_{0} \boldsymbol{E}^{2} / 2\right\rangle}{3 n T / 2}=\frac{1}{3 n} \int \frac{4 \pi k^{2} \mathrm{~d} k}{(2 \pi)^{3}} \frac{1}{1+k^{2} \lambda_{\mathrm{D}}^{2} / S^{2}(k a)}$,

where $S(k a)$ is the Fourier transform of the shape of the superparticles and $a$ the characteristic size of the superparticles (in our case $\left.a \sim X_{0}\right) ; S(k a)$ tends to 1 as $k^{-1} \gg a$.

Equation (22) cannot be used as such for a real plasma of point particles $(S(k a)=1)$ because it includes the electric field at arbitrarily small distances from the charge, which has an infinite energy. It leads to Eq. (20) only if a truncation at small distances is performed, for example $k<\left(\alpha \lambda_{\mathrm{D}}\right)^{-1}$ with $\alpha$ any constant: $\left\langle\epsilon_{0} \boldsymbol{E}^{2} / 2\right\rangle$ in Eq. (20) is then the energy in the electric field for wavelengths larger than $\alpha \lambda_{\mathrm{D}}$. Alternatively and to avoid a cutting procedure, we note that the electric field in Eq. (20) can be taken as the total field produced by the particles to maintain the screening Debye clouds (the polarization electric field; see Callen 2006, Sect. 1.1).

In the case of a PIC plasma all processes at scales below the grid size $a=X_{0}$ are ignored, so that the upper bound of
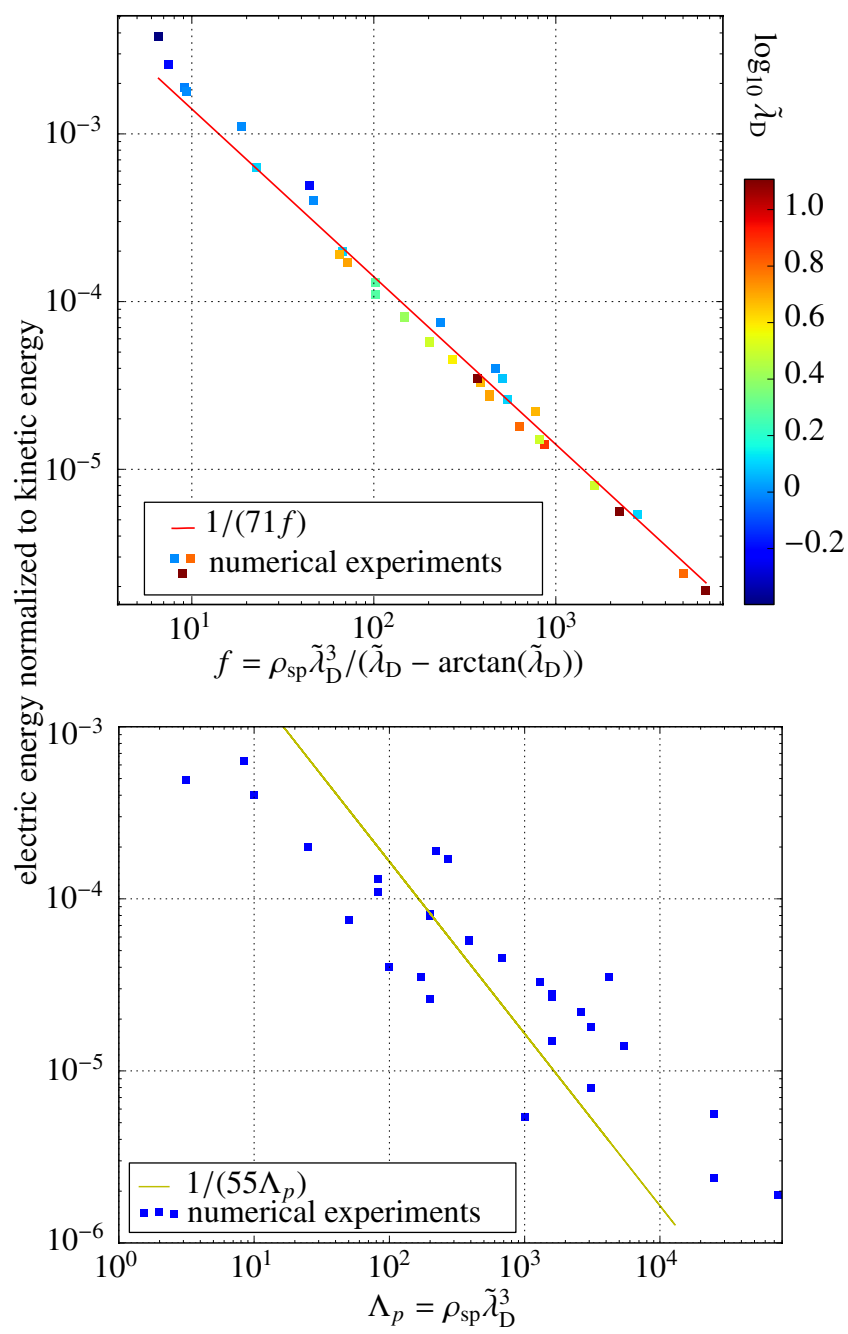

Fig. 11. Top: field energy levels as a function of $f=\rho_{\text {sp }} \tilde{\lambda}_{\mathrm{D}}^{3} /\left(\tilde{\lambda}_{\mathrm{D}}-\right.$ $\left.\arctan \tilde{\lambda}_{\mathrm{D}}\right)$. The colorbar is $\log _{10}\left(\tilde{\lambda}_{\mathrm{D}}\right)$. Blue points at low $f$ have an under-resolved Debye length that could explain the mismatch with $1 / f$. Bottom: field energy levels versus $\Lambda_{p}=\rho_{\mathrm{sp}} \tilde{\lambda}_{\mathrm{D}}^{3}$. We clearly see the mismatch between $1 / \Lambda_{p}$ and the results, even if the trend is correct. The large scatter is a hint that $\Lambda$ is not a relevant parameter to describe field fluctuations. Top and bottom: each point is the result from a simulation. The field energy levels are measured as the energy in the $x$ electric field, $\alpha^{-1} \int \mathrm{d} V e_{x}^{2} / 2$ (with $\alpha$ from Eq. (A.6)), divided by the kinetic energy of the superparticles, $\sum_{\mathrm{sp}}\left(\gamma_{\mathrm{sp}}-1\right)$.

the integral is $k_{\max }=a^{-1}$ and there is no small scale divergence. Since $S(k a) \sim 1$ for $k \ll a^{-1}$, and given that the integration stops at $k=a^{-1}$, we will assume that $S(k a)=1$.

Changing to spherical coordinates, using $u=\lambda_{\mathrm{D}} k$ and $\left\langle E_{x}^{2}\right\rangle=$ $\left\langle\boldsymbol{E}^{2}\right\rangle / 3$, we arrive at

$\frac{\left\langle\epsilon_{0} E_{x}^{2} / 2\right\rangle}{3 n T / 2}=\frac{1}{18 \pi^{2}} \frac{1}{n \lambda_{\mathrm{D}}^{3}} \int_{u_{\min }}^{u_{\max }} \mathrm{d} u \frac{u^{2}}{1+u^{2}}$.

A primitive of the integral is $u-\arctan (u)$. We use $k_{\max }=1 / X_{0}$ or $u_{\max }=\lambda_{\mathrm{D}} / X_{0}=\tilde{\lambda}_{\mathrm{D}}$, while the largest wavelength is given by the simulation domain size and verifies $u_{\min } \ll u_{\max }$. Consequently, we obtain

$\frac{\left\langle\epsilon_{0} E_{x}^{2} / 2\right\rangle}{3 n T / 2}=\frac{1}{18 \pi^{2}} \frac{\tilde{\lambda}_{\mathrm{D}}-\arctan \tilde{\lambda}_{\mathrm{D}}}{\rho_{\mathrm{sp}} \tilde{\lambda}_{\mathrm{D}}^{3}}$,

in good agreement with the simulations (Fig. 11, top panel), except for the constant factors $18 \pi^{2} \sim 178$ (which can be attributed to an approximate choice of $u_{\max }$ ). 
The two limits are interesting. For a very high resolution, $\tilde{\lambda}_{\mathrm{D}} \gg 1$, the field energy decreases as $1 /\left(\rho_{\mathrm{sp}} \tilde{\lambda}_{\mathrm{D}}^{2}\right)$, which is nontrivial and different from what is expected in a real plasma where it decreases as $1 / \Lambda=1 /\left(n \lambda_{\mathrm{D}}^{3}\right)$. The empirical formula of Hockney (1971), generalized to 3D, would also predict $\varepsilon \propto$ $1 /\left(\rho_{\mathrm{sp}} \tilde{\lambda}_{\mathrm{D}}^{3}\right)$ in the high resolution limit. However, our experiments clearly preclude this dependence, and are compatible with Eq. (24) (see Fig. 11). The presence of a finite superparticle volume and of the grid is retained in our calculation only in the upper bound of the integral: physically speaking, physical processes with $k^{-1}<X_{0}$ are smoothed out. This explains the difference between Eq. (24) and that of a real plasma.

For low resolutions, $\tilde{\lambda}_{\mathrm{D}} \lesssim 1$, an expansion of the arctangent shows that the field energy behaves as $1 /\left(54 \pi \rho_{\mathrm{sp}}\right)$, which is finite and independent of $\tilde{\lambda}_{\mathrm{D}}$. In a real plasma, $\varepsilon$ would go to zero as the screening distance vanishes. That this is not the case here indicates that the screening distance does not vanish, because the finite size of the superparticles also plays the role of the screening mechanism.

\subsection{Comparing the PIC and Vlasov-Maxwell models}

We now highlight some differences between PIC models and kinetic models based on Liouville or Klimontovich equations. To do so, we recall how the Vlasov-Maxwell system is derived from these formalisms.

A plasma is constituted of many charged particles in mutual electromagnetic interaction. Under relativistic conditions, a plasma microstate is fully characterized by the positions and velocities of the $N$ particles and by the value of the fields at all space points (the fields must be treated as independent from the particles because of retarded interactions), plus the necessary boundary conditions. Within the frame of classical electrodynamics, the time evolution of a microstate is described by Maxwell equations and by the equations of motion for the particles under the action of the Lorentz force:

$$
\begin{aligned}
& \frac{\mathrm{d}}{\mathrm{d} t}\left(\gamma_{j} \boldsymbol{v}_{j}\right)=\frac{q_{j}}{m_{j}}\left(\boldsymbol{e}_{\mathrm{m}}+\frac{\boldsymbol{v}_{j}}{c} \wedge \boldsymbol{b}_{\mathrm{m}}\right), \\
& \frac{\mathrm{d}}{\mathrm{d} t} \boldsymbol{x}_{j}=\boldsymbol{v}_{j}, \\
& \frac{\partial \boldsymbol{b}_{\mathrm{m}}}{\partial t}=-c \nabla \wedge \boldsymbol{e}_{\mathrm{m}}, \\
& \frac{\partial \boldsymbol{e}_{\mathrm{m}}}{\partial t}=c \nabla \wedge \boldsymbol{b}_{\mathrm{m}}-\frac{1}{\epsilon_{0}} \sum_{j=1}^{N} q_{j} \boldsymbol{v}_{j} \delta\left(\boldsymbol{x}-\boldsymbol{x}_{j}\right), \\
& \nabla \cdot \boldsymbol{e}_{\mathrm{m}}=\frac{1}{\epsilon_{0}} \sum_{j=1}^{N} q_{j} \delta\left(\boldsymbol{x}-\boldsymbol{x}_{j}\right), \\
& \nabla \cdot \boldsymbol{b}_{\mathrm{m}}=0 .
\end{aligned}
$$

Here, $c$ is the speed of light, $\boldsymbol{e}_{\mathrm{m}}$ the microscopic electric field; $\boldsymbol{b}_{\mathrm{m}}=c \boldsymbol{B}_{\mathrm{m}}$ is $c$ times the microscopic magnetic field, $q_{j}, m_{j}, \gamma_{j}$, $\boldsymbol{v}_{j}$, and $\boldsymbol{x}_{j}$ the charge, mass, Lorentz factor, velocity, and position of the particle number $j$. We also define its momentum $\boldsymbol{p}_{j}=$ $m_{j} \gamma_{j} \boldsymbol{v}_{j}$.

At a given time $t$, a microstate is represented by a point $\left\{\boldsymbol{x}_{j}, \boldsymbol{p}_{j}\right\}_{j=1 \ldots N}$ in the $6 N$-dimensional phase-space, and by the fields. One can then consider the collection of microstates having the same macroscopic properties (which can depend on what one is looking for), place them as points in the $6 \mathrm{~N}$ dimensional phase-space, and define the $N$-particle distribution function $f_{N}\left(t,\left\{\boldsymbol{x}_{j}, \boldsymbol{p}_{j}\right\}_{j=1 \ldots N}\right)$ as the number density, at a given time, of these microstates in the $6 N$-dimensional phase-space.
Given that the number of microstates in phase-space is chosen as a continuum, $f_{N}$ is a smooth function (Klimontovich 1982; Nicholson 1983). It defines a macrostate, i.e., an ensemble average of a collection of compatible microstates. The dynamic evolution is then obtained by the Liouville equation, which states that the number of microstates is conserved

$\frac{\partial f_{N}}{\partial t}+\sum_{i=1}^{N} \boldsymbol{v}_{i} \cdot \frac{\partial f_{N}}{\partial \boldsymbol{x}_{i}}+\sum_{i=1}^{N} \dot{\boldsymbol{p}}_{i} \cdot \frac{\partial f_{N}}{\partial \boldsymbol{p}_{i}}=0$

supplemented by Maxwell equations for the microscopic fields $\boldsymbol{e}_{\mathrm{m}}$ and $\boldsymbol{b}_{\mathrm{m}}$ with for sources the particles of the corresponding microstate.

When there is no background magnetic field, and when the particles are non-relativistic, the magnetic field remains negligible toward the electric field which can be computed directly from the particle positions at time $t$ with the use of the electrostatic potential $V(r)=q^{2} /\left(4 \pi \epsilon_{0} r\right)$ (the Coulomb model). The Liouville equation can then be transformed to the infinite BBGKY hierarchy. The first BBGKY level involves the oneparticle distribution function $f_{1}(t, \boldsymbol{w})$ (with $\boldsymbol{w}=(\boldsymbol{x}, \boldsymbol{p})$ ) and the two-point distribution function $f_{2}$ via the correlation function $g_{2}\left(t, \boldsymbol{w}_{1}, \boldsymbol{w}_{2}\right)=f_{2}\left(t, \boldsymbol{w}_{1}, \boldsymbol{w}_{2}\right)-f_{1}\left(t, \boldsymbol{w}_{1}\right) f_{1}\left(t, \boldsymbol{w}_{2}\right)$ :

$$
\begin{aligned}
& \frac{\partial f_{1}}{\partial t}+\boldsymbol{v}_{1} \cdot \frac{\partial f_{1}}{\partial \boldsymbol{x}}-\frac{\partial}{\partial \boldsymbol{x}_{1}} \bar{V}_{t, \boldsymbol{x}_{1}}\left[f_{1}\right] \cdot \frac{\partial f_{1}}{\partial \boldsymbol{p}}=C\left[g_{2}\right], \\
& \bar{V}_{t, \boldsymbol{x}_{1}}\left[f_{1}\right]=\int \mathrm{d}^{6} \boldsymbol{w}_{2} V\left(\left|\boldsymbol{x}_{1}-\boldsymbol{x}_{2}\right|\right) f_{1}\left(t, \boldsymbol{w}_{2}\right) .
\end{aligned}
$$

Here $C$ is an integral operator, vanishing with $g_{2}$. The quantity $\bar{V}_{t, x_{1}}\left[f_{1}\right]$ is the mean potential due to the particle distribution $f_{1}$. Since $f_{1}(\boldsymbol{w})$ is the probability of finding a particle near $\boldsymbol{w}$ independently of the positions of all others, this potential does not include short-range correlations, but only long-range collective effects. It is a macroscopic quantity, just as the fields entering into the Vlasov-Maxwell system (Eq. (28)).

Approximations can then be made to truncate the BBGKY hierarchy. Evaluations of the right-hand side of Eq. (27a) can lead, depending on the hypothesis made, to Boltzmann, Landau, Lenard-Balescu, or more refined kinetic equations. For a fully ionized plasma, the relevant approximation parameter is the number of particles per Debye sphere, or plasma parameter $\Lambda$. For large $\Lambda$, collisions and correlations between small numbers of particles are negligible toward interactions of particles with the fields collectively generated by all others. Keeping only these collective interactions is equivalent to a truncation of BBGKY hierarchy at its lowest level, i.e., $g_{2}=0$, and leads to the VlasovMaxwell system. Generalized to relativistic plasmas ${ }^{5}$ and to two species, it reads

$$
\begin{aligned}
& \frac{\partial f_{1, s}}{\partial t}+\boldsymbol{v} \cdot \frac{\partial f_{1, s}}{\partial \boldsymbol{x}}+q_{s}(\boldsymbol{e}+\boldsymbol{v} \wedge \boldsymbol{b}) \cdot \frac{\partial f_{1, s}}{\partial \boldsymbol{p}}=0, \quad s=\mathrm{i}, \mathrm{e}, \\
& \frac{\partial \boldsymbol{b}}{\partial t}=-c \nabla \wedge \boldsymbol{e} \\
& \frac{\partial \boldsymbol{e}}{\partial t}=c \nabla \wedge \boldsymbol{b}-\frac{1}{\epsilon_{0}} \sum_{s=\mathrm{i}, \mathrm{e}} \iiint \mathrm{d}^{3} \boldsymbol{p} q_{s} \boldsymbol{v} f_{1, s}(t, \boldsymbol{x}, \boldsymbol{p}) \\
& \nabla \cdot \boldsymbol{e}=\frac{1}{\epsilon_{0}} \sum_{s=\mathrm{i}, \mathrm{e}} \iiint \mathrm{d}^{3} \boldsymbol{p} q_{s} f_{1, s}(t, \boldsymbol{x}, \boldsymbol{p}), \\
& \nabla \cdot \boldsymbol{b}=0
\end{aligned}
$$

5 The relativistic Vlasov-Maxwell system is usually derived by using the Klimontovich formalism, not the Liouville formalism (see, e.g., Nicholson 1983; Klimontovich 1982). 


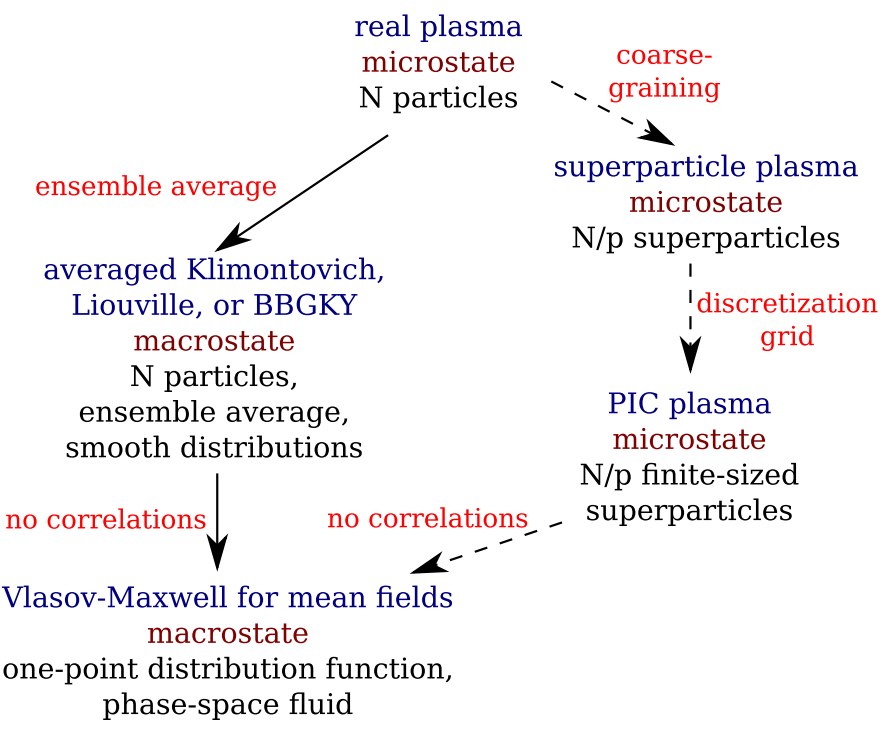

Fig. 12. Different plasma models. Dashed arrows are transitions showing non-trivial effects. Coarse-graining and discretization are discussed in Sects. 5.1 to 5.3 .

with $f_{1, s}(t, \boldsymbol{x}, \boldsymbol{p})$ the one-particle distribution function, for electrons $(s=\mathrm{e})$ or ions $(s=\mathrm{i})$, defined from $f_{N}$. It is also a smooth function, and the Vlasov-Maxwell system describes the evolution of a continuous fluid in the six-dimensional phasespace, where information on the individual nature of the particles has been smoothed out. In particular within this description, the plasma parameter $\Lambda$ is infinite, the fluctuation- and collisioninduced thermalization time is infinite, and the level of electric field fluctuations $\varepsilon$ is zero. We note that these are analytical properties. Numerical solutions of the Vlasov-Maxwell-system will also not strictly recover the collisionless behavior of the plasma. For instance, numerical diffusion arising necessarily from the discretization of Eq. (28) will also lead to a finite thermalization time. We are, however, not aware of a comprehensive study of such effects for algorithms solving the discretized VlasovMaxwell system.

In contrast, the models underlying PIC simulations follow a different path, illustrated in Fig. 12. It consists in following the time evolution of the microstate constituted by $N / p$ superparticles and the fields, Eqs. (2a)-(2e), with $p$ reaching $10^{10}$ or more. One of the consequences is that the PIC plasma has a plasma parameter $\Lambda_{p}=\Lambda / p$ (from Eq. (14)) far smaller than that of the real plasma, so that it includes relatively large correlation and noise levels.

\subsection{Higher-order effects of coarse-graining}

We have highlighted that the coarse-graining step, the description of a real plasma of $N$ particles by a PIC plasma of $N / p$ superparticles, each containing $p$ real particles, involves a reduction of the plasma parameter $\Lambda$ by a factor $p$. This is the main effect of coarse-graining. Higher-order effects arise because after coarse-graining, the model ignores the internal dynamics and correlations of the particles contained within a superparticle.

This can be seen by writing explicitly the grouping: we label the particles either by $w_{n}, n=1 \ldots N$ (with $w=(\boldsymbol{x}, \boldsymbol{p})$ ), or by $w_{i j}$ with $i=1 \ldots N / p$ representing the group number, and $j=1 \ldots p$ the particle number within this group. We denote by $\bar{w}_{\mathrm{sp}, i}$ the position and velocity of the center-of-mass of the group number $i$.
The $N$-particle distribution function $f_{N}$ of the real plasma can then be written formally as

$$
\begin{aligned}
& f_{N}\left(t, w_{1}, \ldots, w_{N}\right)=g_{\mathrm{corr}}\left(t, w_{1}, \ldots, w_{N}\right) \\
& \quad+f_{N / p}\left(t, \bar{w}_{\mathrm{sp}, 1}, \ldots, \bar{w}_{\mathrm{sp}, N / p}\right) \times \prod_{i=1}^{N / p} f_{\mathrm{sp}, i}\left(t, w_{i 1}, \ldots, w_{i p}\right) .
\end{aligned}
$$

This equation introduces $f_{N / p}\left(t, \bar{w}_{\mathrm{sp}, 1}, \ldots, \bar{w}_{\mathrm{sp}, N / p}\right)$, the analog of $f_{N}$ but for the center-of-mass of the particle groups (i.e., of the superparticles); $f_{\mathrm{sp}, i}\left(t, w_{i 1}, \ldots, w_{i p}\right)$, the distribution function of the particles contained within a group, which represents the dynamics and correlations between particles of the same group; and $g_{\text {corr }}\left(t, w_{1}, \ldots, w_{N}\right)$, the correlations ignored by writing $f_{N}=$ $f_{N / p} \times f_{\mathrm{sp}, 1} \ldots f_{\mathrm{sp}, N / p}$, i.e., the correlations between particles of different groups. The PIC approximation then consists in setting $g_{\text {corr }}=0$ and $f_{\mathrm{sp}, i}\left(t, w_{i 1}, \ldots, w_{i p}\right)=$ const.

A complete understanding of these approximations would require developing a BBGKY hierarchy from Eq. (29) and making explicit the electric and magnetic field contributions from the particle groups. This is a complex task. We can, however, stress important consequences of the assumption $f_{\mathrm{sp}, i}\left(t, w_{i 1}, \ldots, w_{i p}\right)=$ const.:

- The superparticles are assumed incompressible. The compressibility due to particle motion within a particle group is thus absent from the coarse-grained plasma.

- The velocity dispersion of the particles within a group is ignored in the coarse-grained plasma. The kinetic pressure resulting from this dispersion is thus also absent.

- The electric fields present in the PIC plasma are computed from the superparticles. This is equivalent to saying that they are computed by taking into account only the monopole distribution of charge created by the internal arrangement of the particles within a group, with higher-order multipole terms neglected. The same holds for the magnetic field.

\section{Discussion and conclusion}

\subsection{Code validation}

We have presented our particle-in-cell code Apar-T (Sect. 2 and Appendix A), and studied several validation tests.

Computation of the spectra from a magnetized or unmagnetized thermal plasma at rest (Sect. 3.1) has proven very accurate, with the plasma pulsation and the right and left cutoff pulsations precisely recovered (Figs. 1 and 2), even in cases where the Debye length and the Larmor radius are not resolved. This proves that the description of individual particle motions in a constant magnetic field and of collective particle dynamics is accurate, and robust with respect to numerical resolution. In particular we showed that Larmor orbits in a constant magnetic field are well described provided that the cyclotron pulsation is well resolved, independently of the grid size. We found, however, a numerical instability with abnormal behavior in the energy curves and high noise levels for under-resolved Debye length or under-resolved Larmor radius, so that this parameter range should be avoided.

Simulations of the filamentation instability (Sect. 3.2) showed a good agreement with linear cold theories, provided that the growth rates are computed from the temporal evolution of the Fourier modes. Discrepancies with theory then range from $5 \%$ to $13 \%$, and can be explained by the wavenumber dependence of the growth rates. On the other hand, the linear growth rates derived from the total energy curves, or equivalently from 
the sum of all Fourier modes, present larger discrepancies with linear theory, ranging between $12 \%$ to $61 \%$. This is explained by the high level of fluctuations in PIC codes that prevent the fastest growing modes to dominate the total energy before the end of the linear phase of the instability.

Simulations of the tearing instability in a relativistic pair plasma gave linear growth rates within $8 \%$ of those found by Pétri \& Kirk (2007) with an analytical linear Vlasov-Maxwell solution, a result not varying significantly when changing the numerical resolution (Sect. 3.4, Fig. 8, and Table 4). This example, where the shape of the velocity distribution is a key feature, is thus in agreement with the Vlasov-Maxwell description. It also validates the new method used to load the relativistic (in both temperature and bulk velocity) Maxwell-Jüttner distribution that we present in Sect. 4 and Appendix D, as well as the general relations used for the relativistic Harris equilibrium (Appendix C). We note that in this case the total energy curves can be used for evaluation of the linear growth rates because the linear phase spans several orders of magnitude in field intensity, so that the fastest growing modes have enough time to dominate the energy.

All in all, these tests show that our code Apar-T is a sound basis for future explorations. They also serve as a base to explore important questions regarding the nature of a PIC plasma, that we summarize in the next section.

\subsection{PIC and real plasmas}

The widespread use of PIC codes for studying plasmas out of equilibrium calls for a deep understanding of the PIC model, and of its relation with a real plasma and with the Vlasov-Maxwell description. Section 5 attempted to provide some explanations.

We have seen that the PIC model lies on two building blocks. The first stems from the capability of computers to handle only up to $\sim 10^{10}$ particles, while real plasmas contain from $10^{4}$ to $10^{20}$ particles per Debye sphere. This means that a coarsegraining step must be used, whereby of the order of $p \sim 10^{10}$ real particles are represented by a single computer superparticle. The second step is field storage on a grid with its subsequent finite superparticle size.

We have introduced the notion of coarse-graining dependent quantities, i.e., physical quantities depending on $p$. The prototype of such quantities is the plasma parameter $\Lambda$, that behaves as $\Lambda_{p} \propto 1 / p$. This vast reduction of $\Lambda$ induces higher noise levels and correlations, but we have again seen that it does not threaten plasma and collisionless behavior as long as $\Lambda_{p}$ remains above unity. All coarse-graining dependent quantities can be expressed as the product of a fluid quantity (which is coarse-graining independent) and a parameter expressing a number of particles per fluid volume. Examples of such parameters include $\Lambda=n \lambda_{\mathrm{D}}^{3}$, $n\left(c / \omega_{\text {pe }}\right)^{3}, n\left(c / \omega_{\text {pi }}\right)^{3}, \ldots$ Their behavior in the PIC plasma can be guessed by taking into account the reduction by a factor $p$ of the number of particles, leading for example to the substitution $\Lambda \rightarrow \Lambda_{p}=\Lambda / p$ in the relevant analytical expressions. We checked this for the collision and fluctuation induced thermalization time (Sect. 5.2), which is indeed proportional to the number of computer superparticles per cell; the lower the number the shorter the thermalization time. Bret et al. (2013) similarly reduce the parameter $n\left(c / \omega_{\mathrm{pe}}\right)^{3}$ by a factor $p$ when applying their theory for the magnetic fluctuation level in a drifting plasma to their PIC simulations. However, the substitution $\Lambda \rightarrow \Lambda_{p}$ is strictly valid only for point-size particles, and the large finite size of the superparticles, which reaches a fraction of a Debye length, suppresses interactions and fluctuations at shorter wavelengths and modifies these scalings. We have detailed how this works for the electric field fluctuation level in a thermal plasma in Sect. 5.3.

We stress that the reduction of the collision and fluctuation induced thermalization time and of other related timescales (e.g., the slowing-down time of fast particles), by 10 or more orders of magnitude, can have important consequences for the relevance of simulations: one has to insure that collisionless kinetic processes remain more efficient than the artificially enhanced collisional and fluctuation induced PIC effects. Similarly, we have seen in Sect 3.2 that the high level of fluctuations alter the linear spectrum of instabilities by preventing the fastest growing modes to dominate the total energy.

A more subtle effect of coarse-graining is due to the loss of the dynamics of the $p$ particles represented by each superparticle. We intuitively expect that it will lead to the overall loss of compressibility due to superparticle incompressibility, of the contribution to kinetic pressure of the particle velocity spreading within a superparticle, and of the multipole contribution to the electric and magnetic fields created by the distribution of particles within a superparticle (see Sect. 5.5). The relevance of these missing effects remains unclear.

\subsection{PIC and Vlasov-Maxwell plasmas}

We have highlighted in Sect. 5.4 that a PIC algorithm simulates a plasma of finite-sized charges in their self-fields, and does not strictly solve the Vlasov-Maxwell system. Using the Vlasov equation assumes that the plasma is represented in phase space by a continuous fluid. In this limit of an infinite number of particles, the plasma parameter $\Lambda$ and the thermalization time are infinite, and the collision frequency and the thermal field fluctuation levels are zero. Using a PIC algorithm amounts to dividing the continuous phase space fluid into discrete elements, and to following their orbits. In this sense, one can say that we integrate the characteristics of the Vlasov equation. However, the newly introduced graininess (which is far higher than that of the original plasma) implies the presence of binary collisions and of correlations between superparticles that is not easy to evaluate, in part because they are reduced by the finite size of the superparticles and the subsequent vanishing of the two-point force at short distances (see Sect. 5.1). The intricate dependence of Eq. (24) is a hint to this complexity. We note that Birsdall \& Langdon (1985, Chap. 12) have derived a generalization of the Balescu-Guernsey-Lenard kinetic equation that includes the use of a grid (and thus of finite sized superparticles), and of the discretization in space and time of the equations. The correlations just mentioned are partly present in this equation, but difficult to extract.

These differences between PIC and Vlasov-Maxwell plasmas are especially enhanced in the linear phase of instabilities. We see two main points. The first is that nonlinear effects absent from the linear theory, and possibly enhanced by the high noise level of the simulation (Sect. 5.3), may have visible consequences (Birsdall \& Langdon 1985, Sect. 13.6; Dieckmann et al. 2006). This example is reported by Daughton (2002) in the context of the drift kink instability of a current sheet: the instability is found to grow faster than predicted by the linear Vlasov theory because the early development of another instability quickly produces nonlinear effects. Bret et al. (2010, Fig. 23) also report significant early nonlinear behavior in counter-streaming situations. We have also reported the presence of field components due to nonlinear effects in Fig. 4.

The second point is that the high level of fluctuations delays the dominance of the fastest growing Fourier modes over 
the sum of other modes. The consequence is that effective linear growth rates measured from total energy curves appear slower than the growth rates of the fastest modes. This is even more important in instabilities where the linear phase is short, and explains the differences between the effective growth rates and the linear cold theory of the counter-streaming instability measured in Sect. 3.2, with discrepancies reaching $60 \%$ or more. It may also explain the differences between theory and measured growth rates of Cottrill et al. (2008), Dieckmann et al. (2006), Haugboelle et al. (2013) for the counter-streaming instability. On the other hand, the differences can be small if the linear phase lasts long enough for the fastest mode to dominate the energy, as is the case for the relativistic tearing instability in Sect. 3.4 or for the Weibel instability of Markidis et al. (2010).

\subsection{Modeling astrophysical plasmas}

In the light of what has been said so far one may wonder what this all implies for the modeling of astrophysical plasmas. We attempt to give some answers in the following. We have shown that the PIC description of an astrophysical plasma bears some risk because the plasma parameter $\Lambda$ is always underestimated, leading to systematic errors in the evaluation of important parameters of the plasma such as the collision and fluctuation induced thermalization time. We stress that this does not lessen the important role of PIC algorithms for deepening our understanding of plasma physics. In particular they also have their virtues, for instance the consideration of certain correlations and of direct particle encounters (with the restrictions discussed in Sect. 5). They provide an accurate description of collisionless kinetic processes such as instabilities, and of the induced turbulence and eventual associated thermalization relevant to collisionless environments.

The Vlasov-Maxwell equations perfectly describe a plasma free of collisions and fluctuations. However, their discretization will again introduce different plasma characteristics. A thorough discussion of these effects is still missing. On the other hand, a collisionless description of astrophysical plasmas is not always correct. On larger spatial and temporal scales, the description of flows and the propagation of non-thermal particles must include collisions to a certain degree. In this regime other models, and in particular Fokker-Planck models, have been shown to give a good description of the plasma and have provided significant results. However, Fokker-Planck models have their own drawbacks, notably that they are local and use dragging and diffusion coefficients not self-consistently derived.

These discrepancies between real, PIC, and Vlasov-Maxwell plasmas are complex, and it needs to be discussed in further detail under what circumstances which model and which numerical realization comes closest to a real plasma. In the long term, it may be justified to use models including correlations in a more systematic way, for example the Landau or the LenardBalescu equation on the theoretical side, and $\mathrm{P}^{3} \mathrm{M}$ algorithms on the numerical side (that include short-range particle-particle interactions Hockney \& Eastwood 1988). Both approaches should then be faced with results from well-controlled collisionless plasma experiments, which are presently in their infancy (see, e.g., Grosskopf et al. 2013).

Acknowledgements. We would like to thank Jérôme Pétri for information and discussions about linear growth rates. We also thank the anonymous referee for comments that allowed to significantly improve the manuscript. We acknowledge support from the French Stellar Astrophysics Program PNPS. Some of the simulations have been performed at pôle scientifique de modélisation numérique, PSMN, at ENS-Lyon, whose staff we thank for their steady technical support. Larger simulations were performed using HPC ressources from GENCI (Grand Équipement National de Calcul Intensif) at CINES and CCRT, under the allocation $\mathrm{x} 2012046960$.

\section{Appendix A: Numerical implementation}

This Appendix is the direct continuation of Sect. 2.

\section{A.1. Computation of the current}

Before going through the normalization of Eqs. (2a)-(2e), we have to understand how the current is computed. As said in Sect. 2.2.2, interpolation of particle quantities to grid nodes is done by attributing to the superparticles a finite volume $V_{\mathrm{sp}}=$ $X_{0}^{3}$. We consider a superparticle, and the cell that contains its center. At time $t$, the superparticle occupies a volume $V_{t}$ of the cell. The charge in the cell is given by $Q_{\text {cell }}(t)=q_{\mathrm{sp}} \times\left(V_{t} / V_{\mathrm{sp}}\right)$. The charge continuity equation then gives

$$
\oiint_{\text {cell }} \boldsymbol{j} \cdot \mathrm{d} \boldsymbol{S}=-\frac{\mathrm{d} Q_{\text {cell }}}{\mathrm{d} t}=\frac{V_{t+\mathrm{d} t}-V_{t}}{V_{\mathrm{sp}}} \frac{q_{\mathrm{sp}}}{\mathrm{d} t} .
$$

The superparticle volume necessarily intersects three faces of the cell that contains its center: one of perpendicular along $\boldsymbol{x}$, one along $\boldsymbol{y}$, and one along $\boldsymbol{z}$. Consequently, the motion of this superparticle will create a current through these three faces, and we can write

$$
\oiint_{\text {cell }} \boldsymbol{j} \cdot \mathrm{d} \boldsymbol{S}=j_{x} X_{0}^{2}+j_{y} X_{0}^{2}+j_{z} X_{0}^{2} .
$$

We have to know which part of the volume variation $V_{t+\mathrm{d} t}-V_{t}$ is attributed to each part of the current. The displacement of the superparticle between $t$ and $t+\mathrm{d} t$ is denoted $(\Delta x, \Delta y, \Delta z)$. The volume variation depends on this displacement, and the part of it proportional to $\Delta x$ is attributed to $j_{x}$, and similarly for the $y$ and $z$ components. More specifically, we can write $V_{t+\mathrm{d} t}-V_{t}=$ $A_{x} \Delta x+A_{y} \Delta y+A_{z} \Delta z$. The areas $A_{i}$ can be evaluated with some geometry (see Sect. A.3.4). Then (and similarly for $y$ and $z$ ):

$j_{x}=-\frac{1}{X_{0}^{2}} \frac{q_{\mathrm{sp}}}{\mathrm{d} t} \frac{A_{x} \Delta x}{V_{\mathrm{sp}}}$.

This way of computing the current ensures that the discrete charge conservation equation is fulfilled, and justifies the advection of the divergence of the fields.

\section{A.2. Normalization}

The problem is formulated with as many equations as variables (Eqs. (2a)-(2e), variables $\boldsymbol{e}, \boldsymbol{b}, \boldsymbol{j}, \boldsymbol{x}_{\mathrm{sp}}$, and $\boldsymbol{v}_{\mathrm{sp}}$ for each superparticle), and it is possible to normalize the equations in a way independent of any physical quantity. We denote normalized quantities with a tilde.

We choose to normalize lengths by $X_{0}$. Consequently, the normalized step-size is unity. Times are normalized by $T_{0}=$ $X_{0} / c$, and velocities are then naturally normalized to $X_{0} / T_{0}=c$. For the fields $\boldsymbol{E}$ and $\boldsymbol{B}$, we use $\boldsymbol{e}=\boldsymbol{E}$ and $\boldsymbol{b}=c \boldsymbol{B}$. These last two quantities are normalized by $e_{0}=b_{0}=m_{\mathrm{e}} c^{2} /\left(e X_{0}\right)$. With this, Eqs. (2a), (2b), and (2c) transform into

$$
\begin{aligned}
& \frac{\mathrm{d} \tilde{\boldsymbol{x}}}{\mathrm{d} \tilde{t}}=\tilde{\boldsymbol{v}} \\
& \frac{\mathrm{d} \gamma \tilde{\boldsymbol{v}}}{\mathrm{d} \tilde{t}}=\left[\frac{m_{\mathrm{e}}}{m_{s}} \frac{q_{s}}{e}\right](\tilde{\boldsymbol{e}}+\tilde{\boldsymbol{v}} \wedge \tilde{\boldsymbol{b}}), \\
& \frac{\partial \tilde{\boldsymbol{b}}}{\partial \tilde{t}}=-\tilde{\nabla} \wedge \tilde{\boldsymbol{e}}
\end{aligned}
$$


with $m_{s}=m_{\mathrm{e}}$ or $m_{\mathrm{i}}$ and $q_{s}=-e$ or $e$ ( $e$ is positive).

For the current $j$, the algorithm computes the quantity $A_{x} \Delta x / V_{\text {sp. }}$. Writing for example the $x$ component of Eq. (2d) gives

$\frac{\partial \tilde{e_{x}}}{\partial \tilde{t}}=(\tilde{\nabla} \wedge \tilde{\boldsymbol{b}})_{x}+\underbrace{\left[\frac{1}{\epsilon_{0}} \frac{e^{2}}{m_{\mathrm{e}} c^{2} X_{0}} p \frac{q_{s}}{e}\right]}_{\alpha}\left[\frac{\tilde{A}_{x} \Delta \tilde{x}}{\tilde{V}_{\mathrm{sp}}}\right] \frac{1}{\tilde{\mathrm{d} t}}$.

Using Eq. (1), we can write

$\alpha=\frac{2 \operatorname{sgn}\left(q_{s}\right)}{n_{x}^{2} \rho_{\mathrm{sp}}^{0}}$,

where sgn is the sign of the charge.

With this, all the equations are completely independent of any physical quantity related to the simulated problem, and depend only on space discretization $\left(n_{x}\right)$ and particle coarsegraining $\left(\rho_{\mathrm{sp}}^{0}\right)$. Time discretization $\left(n_{t}\right)$ will play a role in time integration.

As a final comment, we note that $\rho_{\mathrm{sp}}^{0}$ is a priori unrelated to the actual number of superparticles per cell during the simulation. One should, however, make these two values not too far apart, because the size and timesteps are a fraction $n_{x}$ and $n_{t}$ of the skin depth and plasma period of a $n_{\mathrm{e}}^{0}$-density plasma. If, for example, the superparticle density in the simulation is twice $\rho_{\mathrm{sp}}^{0}$, then $n_{x}$ cells will now represent two skin depths of the $2 n_{\mathrm{e}^{-}}^{0}$ density plasma, and the resolution will decrease. This must be kept in mind in simulations where high density contrasts appear.

\section{A.3. Discrete version of the equations}

In this section we drop the tilde over normalized quantities. We denote the time at which they are considered by a superscript and their spatial location on the grid by a subscript.

\section{A.3.1. The main loop}

The strategy is to use a leap-frog scheme. It has the advantages of being time centered and reversible, and second order in time and space.

Before the loop, $\boldsymbol{b}$ and $\boldsymbol{v}$ are known at time $t-\mathrm{d} t / 2$, and $\boldsymbol{e}$ and $\boldsymbol{x}$ are known at time $t$. This should also be true for the initial conditions, so that initially we integrate backward the velocities and the $\boldsymbol{b}$-field by $-\mathrm{d} t / 2$. Injected particles (if any) should also be correctly staggered.

The structure of the main loop is the following:

1. Half advance of $\boldsymbol{b}^{t-\mathrm{d} t / 2}$ with $\nabla \wedge \boldsymbol{e}^{t} ; \boldsymbol{b}$ is now at time $t$.

2. Update of $\boldsymbol{v}^{t-\mathrm{d} t / 2}$ with $\boldsymbol{b}^{t}$ and $\boldsymbol{e}^{t} ; \boldsymbol{v}$ is now at time $t+\mathrm{d} t / 2$.

3. Update of $\boldsymbol{x}^{t}$ with $\boldsymbol{v}^{t+\mathrm{d} t / 2} ; \boldsymbol{x}$ is now at time $t+\mathrm{d} t$.

4. Half advance of $\boldsymbol{b}^{t}$ with $\nabla \wedge \boldsymbol{e}^{t} ; \boldsymbol{b}$ is now at time $t+\mathrm{d} t / 2$.

5. Boundary for $\boldsymbol{b}$.

6. Full advance of $\boldsymbol{e}^{t}$ with $\nabla \wedge \boldsymbol{b}^{t+\mathrm{d} t / 2} ; \boldsymbol{e}$ is now at time $t+\mathrm{d} t$.

7. Boundary for $\boldsymbol{e}$.

8. Boundary for particles.

9. Computation of the currents from $\boldsymbol{v}^{t+\mathrm{d} t}$ and $\boldsymbol{x}^{t+\mathrm{d} t / 2}$

10. Filtering of the currents.

11. Boundary for the currents.

12. Add currents to $\boldsymbol{e}^{t+\mathrm{d} t}$.

\section{A.3.2. Integration of the fields}

The fields are stored on the grid in a staggered way, with $\boldsymbol{e}$ at the center of the grid edges and $\boldsymbol{b}$ at the center of the grid faces
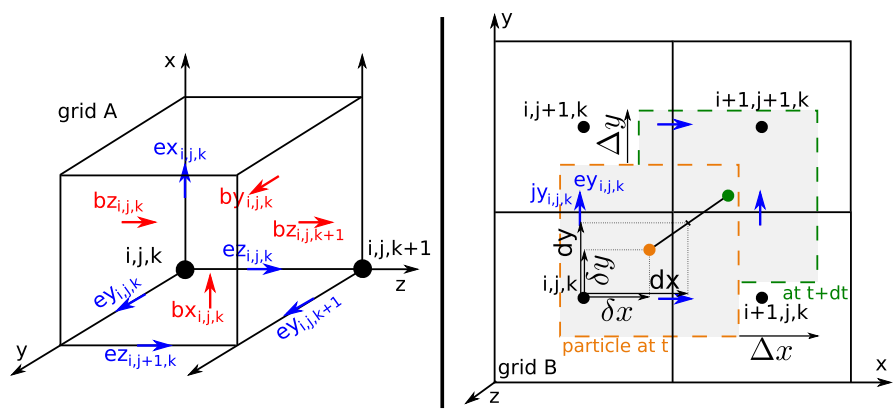

Fig. A.1. Grid and fields locations.

(Fig. A.1). This is the so-called Yee lattice. It allows an easy integration of the fields, and is second-order accurate in time and space (Birsdall \& Langdon 1985, Sect. 15),

$b_{x \mid i, j, k}^{t+\mathrm{d} t / 2}=b_{x \mid i, j, k}^{t-\mathrm{d} t / 2}+\mathrm{d} t\left(e_{y \mid i, j, k+1}^{t}-e_{y \mid i, j, k}^{t}-e_{z \mid i, j+1, k}^{t}+e_{z \mid i, j, k}^{t}\right)$,

and similarly for the other components of $\boldsymbol{b}$ and for $\boldsymbol{e}$.

To reduce the effects of Čerenkov emission (see Appendix B.1), we have also implemented a fourth order solver (Greenwood et al. 2004).

\section{A.3.3. Moving the particles}

Integration of the equation of motion for the superparticles is done with the algorithm described by Birsdall \& Langdon (1985, Sect. 15.4). It is a relativistic generalization of the leap-frog scheme, time centered, time reversible, and second order accurate. We note however that, as pointed out by Vay (2008), it can have shortcomings for ultrarelativistic particles. In short, Eqs. (A.4a) and (A.4b) are discretized as

$$
\begin{aligned}
& \left(\boldsymbol{u}^{t+\mathrm{d} t / 2}-\boldsymbol{u}^{t-\mathrm{d} t / 2}\right) / \mathrm{d} t= \\
& \frac{m_{\mathrm{e}}}{m} \frac{q}{e}\left\{\boldsymbol{e}^{t}+\frac{\boldsymbol{u}^{t+\mathrm{d} t / 2}+\boldsymbol{u}^{t-\mathrm{d} t / 2}}{2 \gamma^{t}} \wedge \boldsymbol{b}^{t}\right\} \\
& \frac{\boldsymbol{x}^{t+\mathrm{d} t}-\boldsymbol{u}^{t}}{\mathrm{~d} t}=\frac{\boldsymbol{u}^{t+\mathrm{d} t / 2}}{\sqrt{1+\left(\boldsymbol{u}^{t+\mathrm{d} t / 2}\right)^{2}}}
\end{aligned}
$$

with $\boldsymbol{u}=\gamma \boldsymbol{v}$ and $\gamma$ the Lorentz factor. Defining $\boldsymbol{u}^{-}=\boldsymbol{u}^{t-\mathrm{d} t / 2}+$ $q m_{\mathrm{e}} /(e m) \boldsymbol{e}^{t} \mathrm{~d} t / 2$ and $\boldsymbol{u}^{+}=\boldsymbol{u}^{t+\mathrm{d} t / 2}-q m_{\mathrm{e}} /(e m) \boldsymbol{e}^{t} \mathrm{~d} t / 2$ and substituting into (A.8a) leads to

$\frac{\boldsymbol{u}^{+}-\boldsymbol{u}^{-}}{\mathrm{d} t}=\frac{q m_{\mathrm{e}} /(e m)}{2 \gamma^{t}}\left(\boldsymbol{u}^{+}+\boldsymbol{u}^{-}\right) \wedge \boldsymbol{b}^{t}$.

This equation is the classical rotation around a $\boldsymbol{b}$ field (Birsdall \& Langdon 1985, Sect. 4.4; Hockney \& Eastwood 1988, Sect. 4.7.1), and is solved via

$\boldsymbol{u}^{+}=\boldsymbol{u}^{-}+\frac{2}{1+\left(\frac{\Omega \mathrm{d} t}{2}\right)^{2}}\left(\boldsymbol{u}^{-}+\boldsymbol{u}^{-} \frac{\Omega \mathrm{d} t}{2}\right) \wedge \Omega$,

with the rotation vector $\Omega=q m_{\mathrm{e}} /\left(2 e m \gamma^{t}\right) \boldsymbol{b}^{t}$. Finally, we use $\gamma^{t}=\sqrt{1+\left(u^{-}\right)^{2}}=\sqrt{1+\left(u^{+}\right)^{2}}$. In Eq. (A.8a), the fields have to be known at time $t$. It explains the need for the half advances of $\boldsymbol{b}$ in the general scheme.

The interpolation of the fields at particle positions is done via a trilinear interpolation. We denote by $(i, j, k)$ the nodes of the 
main grid $A$ (Fig. A.1), and we introduce a second grid $B$ whose cell centers are on $(i, j, k)$. Consider a superparticle at position $x=i+\delta x, y=j+\delta y, z=k+\delta z$. The superparticle is actually a charge cloud of volume equal to a cell, and this volume intersects the cell of the second grid with center $(i, j, k)$ in a volume $V_{i, j, k}=$ $(1-\delta x)(1-\delta y)(1-\delta z)$, the cell of the second grid with center $(i+1, j, k)$ in a volume $V_{i+1, j, k}=\delta x(1-\delta y)(1-\delta z)$, and so on. For a quantity $f$ defined at grid points $(i, j, k)$, the weight associated to $f_{i, j, k}$ is $V_{i, j, k}$, the one associated to $f_{i+1, j, k}$ is $V_{i+1, j, k}$, and so on for a total of 8 points.

However, neither $\boldsymbol{e}$ nor $\boldsymbol{b}$ are defined at grid points $(i, j, k)$ (Fig. A.1), and they must be first interpolated at grid points before applying the above procedure. This is done for example with $f_{i, j, k}=0.5\left(e_{x, i-1, j, k}+e_{x, i, j, k}\right)$ or $f_{i, j, k}=0.25\left(b_{z, i, j, k}+\right.$ $\left.b_{z, i-1, j, k}+b_{z, i-1, j-1, k}+b_{z, i, j-1, k}\right)$. Details can be found in Matsumoto \& Omura (1993); Messmer (2001).

We note that the superparticle shape used for interpolation of fields to particle position and for interpolation of the current to grid nodes is the same. This is required to avoid the existence of a self-force on the superparticles and to conserve the total momentum (Birsdall \& Langdon 1985, Sect. 8.6).

\section{A.3.4. Computation of the current}

The current $\boldsymbol{j}_{i, j, k}$ is defined at the same locations as $\boldsymbol{e}_{i, j, k}$. For current deposition, we again consider the volumes occupied by the superparticle in the grid $B$ cells. As the superparticle moves, these volumes vary. We denote by $(i+\delta x, j+\delta y, k+\delta z)$ the position of the superparticle at $t-\mathrm{d} t$, and we assume that it moves from $(\Delta x, \Delta y, \Delta z)$ between $t-\mathrm{d} t$ and $t$.

Consider, for example, the volume of the superparticle in the cell of center $(i, j, k)$. Its variation is given by $d V=(1-\delta x-$ $\Delta x)(1-\delta y-\Delta y)(1-\delta z-\Delta z)-(1-\delta x)(1-\delta y)(1-\delta z)$. Defining $d_{x}=\delta x+\Delta x / 2, c_{x}=1-d_{x}$, and similarly for $y$ and $z$, one finds

$$
\begin{aligned}
d V= & \Delta x\left[-c_{y} c_{z}-\Delta y \Delta z / 12\right] \rightarrow j_{x \mid i, j, k} \\
& +\Delta y\left[-c_{z} c_{x}-\Delta z \Delta x / 12\right] \rightarrow j_{y \mid i, j, k} \\
& +\Delta z\left[-c_{x} c_{y}-\Delta x \Delta y / 12\right] \rightarrow j_{z \mid i, j, k}
\end{aligned}
$$

As explained in Appendix A.1, the part of (A.11) proportional to the displacement along $x$ is attributed to $j_{x \mid i, j, k}$, and so on.

A similar treatment is done with the cells that intersect the superparticle volume. These are cells centered in $(i+\epsilon, j+\eta, k+\xi)$, with $\epsilon, \eta$ and $\xi$ equal either to 0 or 1 (8 cells). For each of these cells, only the faces intersecting the superparticle volume are concerned, so that in total there are only 12 currents to update.

Currents can be smoothed before being added to $\boldsymbol{e}$. This has the effect of reducing electromagnetic noise (see Appendix B.2). This is done in the following way for the current of cell $(i, j, k)$ : attribute a weight of 1 to cell $(i, j, k)$; of 0.5 to cells $(i \pm 1, j, k)$, $(i, j \pm 1, k)$, and $(i, j, k \pm 1)$; of 0.25 to cells $(i \pm 1, j \pm 1, k),(i \pm$ $1, j, k \pm 1)$, and $(i, j \pm 1, k \pm 1)$; of 0.125 to cells $(i \pm 1, j \pm 1, k \pm 1)$; and normalize the sum of the weights to 1 .

\section{A.4. Boundaries}

Periodic and reflective boundaries are available. The latter simulate a perfect conductor at the domain boundary by imposing the correct values for the electric and magnetic fields $(\boldsymbol{b}=\boldsymbol{e}=0$ inside the conductor, $\boldsymbol{b}_{\text {normal }}=\boldsymbol{e}_{\text {tangential }}=0$ at the conductor surface), and by reflecting the particles.

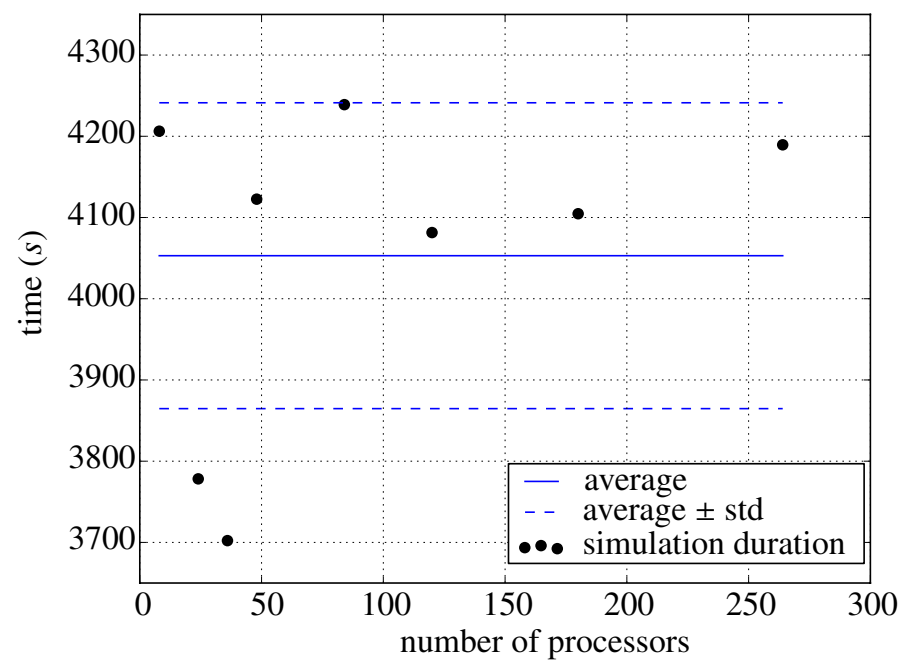

Fig. A.2. Simulation time duration versus number of cores, the spatial domain extending in proportion to the number of cores. The standard deviation corresponds to $4 \%$ of the mean value.

\section{A.5. Parallel efficiency}

The code parallelization was performed and tested by Messmer (2001, Chap. 4). It uses Fortran 90 and MPI. The simulation domain is decomposed in sub-domains of equal length along the $z$ direction, and all the cells and particles of each sub-domain are assigned to a processor. To minimize communications between processors, ghost cells for the fields are added to each sub-domain. Communication between neighboring processors occurs at each step involving the boundaries: for particles leaving or entering the domain, for the fields, and for the currents.

The domain is currently decomposed along one direction only. This is relevant for simulations of collisionless shocks where the domain is elongated along the flow direction, or for 2D magnetic reconnection simulations where the presence of the over-dense current sheet at the domain center would lead to load balancing issues if a 2D domain decomposition were used.

We have tested the efficiency of this implementation with simulations using 16 superparticles per cell and a domain size of $60 \times 60 \times\left(16 n_{\text {proc }}\right)$, where the number of cores varied from $n_{\text {proc }}=16$ to 256 . The corresponding (weak) scaling results, shown in Fig. A.2, are satisfactory. Simulation times scatter with a standard deviation of $4 \%$ around a constant value. The scatter is probably due to the uncontrolled node geometry.

\section{Appendix B: Numerical effects}

We have said in Sect. 5 that passing from a real plasma to a PIC model implies a discretization of the equations. This step comes with numerical issues that have been largely studied by Birsdall \& Langdon (1985) and Hockney \& Eastwood (1988). We highlight part of their work here.

\section{B.1. Local numerical effects}

- Stability of the electric part of the superparticle motion integrator used here requires that $\Omega \Delta t<2$, with $\Omega$ the pulsation of oscillation of the superparticles (usually the plasma pulsation). The magnetic part is unconditionally stable.

- Courant condition for the stability of the field integrator in vacuum is $c \Delta t<X_{0} / \sqrt{2}$. 
- The dispersion relation of electromagnetic waves in vacuum is modified by the grid. This modification depends on the angle of propagation with respect to the grid, and waves can have a phase velocity smaller than $c$ (Greenwood et al. 2004). If superparticles with velocity close to $c$ are present, they can overtake light waves and emit Čerenkov radiation. This results in the production of non-physical fields. The situation can be improved with a higher order interpolation scheme for the fields.

\section{B.2. Global numerical effects}

By considering the algorithm as a whole, Birsdall and Langdon were able to identify numerical effects not predicted by the consideration of subparts alone.

For example, the discrete space representation of the continuous quantities introduces a periodicity in Fourier space of period $k_{0}=2 \pi / X_{0}$ (with $X_{0}$ the grid spacing). A physical mode of wavenumber $k=2 \pi / \lambda$ will then have, in the numerical plasma, aliases of wavenumbers $k+n k_{0}$, and $-k+n k_{0}$, with $n$ an integer. Instabilities can arise if the physical mode couples resonantly with one of the aliases. This coupling cannot occur if $k<-k+k_{0}$, i.e., if $\lambda>2 X_{0}$ (we note that it is Nyquist-Shannon criterion to avoid spectral aliasing). Just as in signal processing, the strength of the aliases can be reduced by low-pass filtering the time-series, and this is what is done by attributing a cloud shape to the superparticles. Aliases are even more reduced when the superparticle shapes have a fast decaying Fourier transform, that is, when they are smoother.

We mention in particular the following effects due to grid aliasing:

- A cold beam of velocity $v_{\text {beam }}$ becomes unstable if the Doppler shifted frequency of Langmuir oscillations is near the grid-crossing frequency $k_{\text {grid }} v_{\text {beam. }}$. The beam is then heated. It is not the case if $\lambda_{\mathrm{D}} / X_{0}>0.046$.

$-\lambda_{\mathrm{D}} / X_{0}>1 / \pi$ is needed to avoid an artificial numerical heating of a Maxwellian plasma. Otherwise, the plasma is heated up to the point where $\lambda_{\mathrm{D}}$ reaches $X_{0} / \pi$.

- The rate of passage of the superparticles through the cell faces produces a high-frequency noise; the rougher the superparticle shapes, the more important is the noise.

Similarly to grid effects, a finite timestep implies that harmonics differing from a multiple of $2 \pi / \Delta t$ are not differentiated by the algorithm, and there are time aliases as well.

- This implies no other instabilities in the case of a nonmagnetized Maxwellian plasma.

- In a magnetized plasma, artificial coupling of cyclotron harmonics can lead to instabilities.

A last point is that the effects of the grid, as well as other errors, act as a random force $F(t)$ on the superparticles. Consequently, the velocity of a superparticle undergoes a random walk, $\mathrm{d} v / \mathrm{d} t \propto$ $F(t)$, and the kinetic energy $\left\langle v^{2}\right\rangle$ increases linearly with time. Hockney \& Eastwood (1988, Sect. 9.2) shows that this is indeed the cause of plasma self-heating in superparticle simulations. This is also what we find in our thermal simulations (see Sect. 3.1.3).

\section{B.3. Qualitative constraints on timestep and sizestep}

- The step-size $X_{0}$ of the grid (which is also roughly the superparticle size), and the time-step $\Delta t$, must be smaller than the scales of the phenomena studied. This scale can be an instability wavelength or growth rate, the cyclotron radius or pulsation, gradient scales, etc.

- The same is true for the mean distance between superparticles: $n_{\mathrm{sp}}^{-1 / 3}<\lambda_{\text {relevant }}$. This is equivalent to having a high enough number of superparticles per volume $\lambda_{\text {relevant }}^{3}$. The case $\lambda_{\text {relevant }}=\lambda_{\mathrm{D}}$ applies to the description of plasma behavior.

- If thermal effects are important, then one should insure that the distribution function $g(\boldsymbol{p})$ is well represented on scales where these effects are important. It requires a high enough number of superparticles per relevant volume. Birsdall \& Langdon (1985, Sect. 15.19) mention that it is sufficient to have a good representation of the relevant projections of $g$.

- The plasma should remain collisionless: the collision time should be greater than relevant timescales (instability growth rates, etc.).

\section{B.4. Limitations for the computation of photon spectra}

- The highest frequency represented is $2 \pi c / X_{0}=2 \pi n_{x} \omega_{\text {pe }}$, so that high energy radiation is absent from the code and must be computed separately to extract photon spectra. This is done for example by Hededal (2005), Trier Frederiksen et al. (2010), Nishikawa et al. (2011), and Cerutti et al. (2012) from superparticle motions, with the inclusion of radiative energy losses. However, even in these cases, effects such as plasma frequency cutoff, Raizin effect, or transition radiation are not described because they are due to the back-reaction of the plasma particles on the electromagnetic waves, waves that are absent from the PIC code and are only computed afterward.

\section{Appendix C: Relativistic Harris current sheet}

Harris configuration is one of the rare fully consistent solutions of the Vlasov-Maxwell system in a non-homogeneous case. Its generalization to the relativistic case is not difficult, and was partly done by Hoh (1966) for a Maxwell-Jüttner distribution and non-relativistic current speeds. The fully relativistic case appeared later for pair plasmas with the same temperature for both species, for example in Kirk \& Skjæraasen (2003) or Pétri \& Kirk (2007). Here, we propose a formulation for the relativistic case with an arbitrary temperature and mass ratio for ions (singly ionized) and electrons.

We define a frame $\mathcal{R}_{0}$, where the magnetic field is assumed to have the dependence given by Eq. (5), and the particles sustaining this field are assumed to have a Maxwell-Jüttner distribution function given in $\mathcal{R}_{0}$ by

$f(\boldsymbol{x}, \boldsymbol{p})=\frac{\mu_{s} n_{s}^{\prime}(x)}{4 \pi K_{2}\left(\mu_{s}\right)} \exp \left\{-\mu_{s} \Gamma_{s}\left(\sqrt{1+p^{2}}-\beta_{s} p_{y}\right)\right\}$,

with $s=\mathrm{i}$ for ions or e for electrons, $\mu_{s}=1 / \Theta_{s}=m_{s} c^{2} / T_{s}$, $\boldsymbol{p}=\gamma \boldsymbol{v} / c$, and $K_{2}$ the modified Bessel function of the second kind; $U_{s}$ is the bulk velocity of species $s$, and $\Gamma_{s}$ the associated Lorentz factor. We note that $f_{s}$ is normalized with respect to $\boldsymbol{p}$ to $\Gamma_{s} n_{s}^{\prime}(x)$, so that $n_{s}^{\prime}$ is the density of species $s$ in its comobile frame (noted with a prime), and $\Gamma_{s} n_{s}^{\prime}$ its density in $\mathcal{R}_{0}$.

Inserting Eq. (C.1) into the Vlasov equation expressed in $\mathcal{R}_{0}$,

$\boldsymbol{v} \cdot \frac{\partial f_{s}}{\partial \boldsymbol{x}}+\frac{q_{s}}{m_{s} c} \boldsymbol{v} \wedge \boldsymbol{B}(\boldsymbol{x}) \cdot \frac{\partial f_{s}}{\partial \boldsymbol{p}}=0$ 
leads to the relation for the comobile number density

$n_{s}^{\prime}(x)=\frac{n_{0, s}^{\prime}}{\cosh ^{2}(x / L)}$

with

$\frac{\Gamma_{s} U_{s}}{c}=-\frac{2 T_{s}}{q_{s} B_{0} L c}=-2 \Theta_{s} \frac{d_{\mathrm{e}}^{\prime}}{L} \frac{\omega_{\mathrm{pe}}^{\prime}}{\omega_{\mathrm{c} s}} \operatorname{sgn}\left(q_{s}\right)$,

with $\operatorname{sgn}\left(q_{s}\right)$ the sign of the charge, $\omega_{\mathrm{c} s}=\left|q_{s}\right| B_{0} / m_{s}, \omega_{\mathrm{pe}}^{\prime}=$ $\sqrt{n_{0}^{\prime} e^{2} /\left(\epsilon_{0} m_{\mathrm{e}}\right)}$, and $d_{\mathrm{e}}^{\prime}=c / \omega_{\mathrm{pe}}^{\prime}$.

We note that the absence of electric field in Eq. (C.2) implicitly assumes that the plasma is quasi neutral in $\mathcal{R}_{0}$, which is true only if the overall charge density in this frame vanishes:

$\Gamma_{i} n_{0, \mathrm{i}}^{\prime}=\Gamma_{\mathrm{e}} n_{0, \mathrm{e}}^{\prime}$.

We now use the Maxwell-Ampère equation in $\mathcal{R}_{0}: \nabla \wedge \boldsymbol{B}=$ $\mu_{0} e\left[\Gamma_{\mathrm{i}} n_{\mathrm{i}}^{\prime}(x) U_{\mathrm{i}}-\Gamma_{\mathrm{e}} n_{\mathrm{e}}^{\prime}(x) U_{\mathrm{e}}\right]$. Insertion of $n^{\prime}(x)$ and $B(x)$ leads, not surprisingly, to a pressure balance between the unmagnetized center of the sheet and the magnetically dominated outer domain:

$\frac{B_{0}^{2}}{2 \mu_{0}}=n_{0, \mathrm{e}}^{\prime} T_{\mathrm{e}}+n_{0, \mathrm{i}}^{\prime} T_{\mathrm{i}}$.

Manipulating Eq. (C.6) and defining $\chi=\left(1+T_{\mathrm{i}} / T_{\mathrm{e}}\right) / 2$ we obtain

$\Theta_{\mathrm{e}}=\frac{1}{4 \chi}\left(\frac{\omega_{\mathrm{ce}}}{\omega_{\mathrm{pe}}^{\prime}}\right)^{2}$.

Given a temperature ratio $\chi$, the four variables $L / d_{\mathrm{e}}^{\prime}, \Theta_{\mathrm{e}}, \omega_{\mathrm{ce}} / \omega_{\mathrm{pe}}^{\prime}$ and $\Gamma_{\mathrm{e}} U_{\mathrm{e}} / c$ are constrained by the two Eqs. (C.4) (for $s=\mathrm{e}$ ) and (C.7). Consequently, one needs to specify two of them. Then, the ion velocity and temperature are easily deduced with Eq. (C.4) for $s=\mathrm{i}$ and with $\chi ; L$ and $\omega_{\text {ce }}$ expressed in units of $d_{\mathrm{e}}$ and $\omega_{\text {pe }}$, that are useful for a setup in a simulation, are then deduced with $\Gamma_{\mathrm{e}}$. A special case is when the temperatures are equal: then $\Gamma_{\mathrm{i}} U_{\mathrm{i}}=-\Gamma_{\mathrm{e}} U_{\mathrm{e}}, n_{\mathrm{e}}^{\prime}=n_{\mathrm{i}}^{\prime}$, and $\Theta_{\mathrm{e}}=(1 / 4)\left(\omega_{\mathrm{ce}} / \omega_{\mathrm{pe}}^{\prime}\right)^{2}$.

\section{Appendix D: A method for loading a drifting Maxwell-Jüttner distribution}

This Appendix directly follows the notations of Sect. 4, and presents a concrete way to load a drifting Maxwell-Jüttner distribution in a PIC code.

Starting from the distribution in Eq. (13), we make a first change of variables $\left(p_{x}, p_{y}, p_{z}\right) \rightarrow(x, y, z)=\left(p_{x} / \gamma_{y}, p_{y}, p_{z} / \gamma_{y}\right)$, where $\gamma_{y}=\sqrt{1+p_{y}^{2}}$, and we then change to cylindrical coordinates $(r, \theta, y)$ with the axis along $y$. We integrate along $\theta$. Finally, a last change of variables $(r, y) \rightarrow(u, y)=\left(\sqrt{1+r^{2}}, y\right)$ leads to the distribution for the random variables $(U, Y) \in[1,+\infty[\times]-$ $\infty,+\infty[$ :

$$
\begin{aligned}
j_{U, Y}(u, y)= & \frac{\mu}{2 K_{2}(\mu) \Gamma_{0}} \gamma_{y} \exp \left\{\mu \Gamma_{0} \beta_{0} y\right\} \\
& \times u \exp \left\{-\mu \Gamma_{0} \gamma_{y} u\right\} .
\end{aligned}
$$

It is then easy to obtain the marginal distribution for $Y$ :

$$
\begin{aligned}
j_{Y}(y) & =\int_{1}^{\infty} \mathrm{d} u j_{U, Y}(u, y) \\
& =\frac{1+\mu \Gamma_{0} \gamma_{y}}{2 \mu \Gamma_{0}^{3} K_{2}(\mu)} \exp \left\{-\mu \Gamma_{0}\left(\gamma_{y}-\beta_{0} y\right)\right\} .
\end{aligned}
$$

From this, we deduce the conditional probability distribution of $U$ given the value $y$ of $Y$ :

$$
j_{U \mid y}(u)=\frac{j_{U, Y}(u, y)}{j_{Y}(y)}=\frac{a_{y}^{2} e^{a_{y}}}{1+a_{y}} u \exp \left\{-a_{y} u\right\},
$$

with $a_{y}=\mu \Gamma_{0} \sqrt{1+y^{2}}$.

Then, for each particle, one has to generate $y=p_{y}$ according to distribution D.2, compute $a_{y}$, and generate $u$ according to distribution D.3.

For the first step, we use the method of the inversion of the cumulative distribution. This method is based on the fact that if $W$ is a uniform random variable on $[0,1]$, if $F(s)=\int_{-\infty}^{s} f(x) \mathrm{d} x$ is the cumulative distribution of the distribution $f$ and $F^{-1}$ its inverse, then $F^{-1}(W)$ follows the distribution $f$. In practice, one has to choose random numbers $w_{i}$ in $[0,1]$, and the $y_{i}=F^{-1}\left(w_{i}\right)$ will be distributed according to $f$.

There is, however, no analytic expression for the cumulative distribution $J(y)=\int_{-\infty}^{y} j_{Y}(z) \mathrm{d} z$. We compute numerically $J^{-1}(t)$ on a grid of points $t_{i}=i / N, i=1 \ldots(N-1)$. For each index $i$, we want to find $y_{i}$ such that $\int_{-\infty}^{y_{i}} j_{Y}(z) \mathrm{d} z=t_{i}$. We thus compute numerically the integral $\int_{-\infty}^{y} j_{Y}(z) \mathrm{d} z$ up to the point where it reaches $t_{i}$, and then attribute the value of $y$ to $y_{i}$. We use the following algorithm:

1. Choose a maximal integration step $\delta y_{\max }$, and set $\delta y=\delta y_{\max }$. Choose a tolerance tol.

2. Start from a low enough value $y_{0}$ such that $j_{Y}\left(y_{0}\right) \ll 1$, and set $y=y_{0}$. Also set $i=1$.

3. Set $J=0$, or if possible $J=\int_{-\infty}^{y_{0}} j_{Y}(z) \mathrm{d} z$.

4. Compute $J=J+\delta y j_{Y}(y)$.

5. $J$ is now an estimation of $\int_{-\infty}^{y} j_{Y}(z) \mathrm{d} z$.

If $\left|J-t_{i}\right|<$ tol, then the desired $y_{i}=J^{-1}\left(t_{i}\right)$ is $y$. Set $i=i+1$, and go back to step 4 .

If $J<t_{i}$, set $y=y+\delta y$, and go back to step 4 .

If $J>t_{i}$, set $\delta y=\delta y / 2$ and go back to step 4 .

We run this algorithm once for the needed $\mu$ and $\Gamma_{0} \beta_{0}$ and store the $y_{i}$ in a file. Then, during the particle initialization, we choose random integers $i$ between 1 and $N-1$ and set $p_{y}=y_{i}$.

Once $y$ is known, we have to pick a $u$ according to Eq. (D.3). Since $a_{y}$ can be anything between $\mu \Gamma_{0}$ and $+\infty$, we cannot generate a file before the program run, and we have to invert $F$ on the flight. It turns out that we can integrate $j_{U \mid y}$. After some basic manipulations, we arrive at

$v=\int_{1}^{u} \mathrm{~d} t j_{U \mid y}(t) \quad \Leftrightarrow \quad l(x)=w l\left(a_{y}\right)$

where $x=a_{y} u, l(x)=(1+x) \exp (-x)$, and $v$ and $w$ are two random numbers between 0 and 1 . Inversion of the right side of Eq. (D.4) is easily done with a Newton method because of the smoothness of the function $l$. Starting from $x=a_{y}$ is a good idea, and one must enforce a minimum number of iterations.

In Fig. 9, we show that the method is accurate.

\section{Appendix E: Analytical expressions for quantities averaged over a Maxwell-Jüttner distribution}

This Appendix details a method used to obtain the analytical expressions of Table 5 for the averaged quantities weighted by a Maxwell-Jüttner distribution. The notations are those in Sect. 4. 
M. Melzani et al.: Apar-T: code, validation, and physical interpretation of particle-in-cell results

\section{E.1. In the comoving frame}

We start with averages over the distribution with zero drift velocity (i.e., the distribution in the frame comoving with the plasma). The calculations are easier when the distribution function is expressed in terms of Lorentz factors $\gamma_{0}$ :

$g_{0}\left(\gamma_{0}\right)=\frac{\mu}{K_{2}(\mu)} \gamma_{0} \sqrt{\gamma_{0}^{2}-1} \exp \left\{-\mu \gamma_{0}\right\}, \quad \gamma_{0} \in[1,+\infty[$.

We introduce two relations ${ }^{6}$ :

$I(\mu) \equiv \int_{1}^{+\infty} \mathrm{d} \gamma_{0} \sqrt{\gamma_{0}^{2}-1} \exp \left\{-\mu \gamma_{0}\right\}=\mu^{-1} K_{1}(\mu)$,

and (DLMF 2013, Eq. (10.29.4))

$\frac{\mathrm{d}}{\mathrm{d} \mu}\left[\mu^{-v} K_{v}(\mu)\right]=-\mu^{-v} K_{v+1}(\mu)$.

The method is then to derive Eq. (E.3) as many times as needed with respect to $\mu$, with the help of Eq. (E.4). For example the integral of $g_{0}$ is easily deduced from $\mathrm{d} I / \mathrm{d} \mu$.

\section{E.2. Drifting distribution}

We now consider the distribution with a drift velocity $\beta_{0} \hat{\boldsymbol{y}}$. We assume that we want the average of a quantity $M$,

$\langle M\rangle=\iiint_{\mathbb{R}^{3}} \mathrm{~d}^{3} \boldsymbol{p} g(\boldsymbol{p}) M(\boldsymbol{p})$,

with $g$ given by Eq. (13). We use the change of variables $p_{0, x}=$ $p_{x}, p_{0, y}=\Gamma_{0}\left(p_{y}-\beta_{0} \sqrt{1+p^{2}}\right), p_{0, z}=p_{z}$, which amounts to passing back into the comoving frame. Using $\mathrm{d}^{3} \boldsymbol{p} / \gamma=\mathrm{d}^{3} \boldsymbol{p}_{0} / \gamma_{0}$, we arrive at

$\langle M\rangle=\Gamma_{0}^{-1} \iiint_{\mathbb{R}^{3}} \frac{\mathrm{d}^{3} \boldsymbol{p}_{0}}{\gamma_{0}} g_{0}\left(\boldsymbol{p}_{0}\right) \gamma M(\boldsymbol{p})=\Gamma_{0}^{-1}\left\langle\frac{\gamma M(\boldsymbol{p})}{\gamma_{0}}\right\rangle_{0}$,

where $\langle\cdot\rangle_{0}$ means that the average is taken with $g_{0}$, and where $\gamma$ and $\boldsymbol{p}$ are to be expressed with comoving quantities (subscript 0 ). With this last formula, one is left with averages in the comoving frame and can use the previous method.

\section{References}

Baumann, G., \& Nordlund, Å. 2012, ApJ, 759, L9

Benedetti, C., Sgattoni, A., Turchetti, G., \& Londrillo, P. 2008, IEEE Trans. Plasma Sci., 36, 1790

Birdsall, C. K. 1967, NASA Special Publ., 153, 375

Birdsall, C. K. 1999, in Dynamical Systems, Plasmas and Gravitation, eds. P. G. L. Leach, S. E. Bouquet, J.-L. Rouet, \& E. Fijalkow, Lect. Notes Phys. (Berlin: Springer Verlag), 518, 383

Birsdall, C., \& Langdon, A. 1985, Plasma Physics via Computer Simulation (New-York: McGraw-Hill)

Bret, A., Gremillet, L., \& Dieckmann, M. E. 2010, Phys. Plasmas, 17, 120501

Bret, A., Stockem, A., Fiuza, F., et al. 2013, Phys. Plasmas, 20, 042102

Buneman, O. 1959, Phys. Rev., 115, 503

Buneman, O. 1976, Comp. Phys. Comm., 12, 21

Burger, P. 1965, J. Appl. Phys., 36, 1938

Bykov, A. M., \& Treumann, R. A. 2011, A\&ARv, 19, 42

6 To evaluate the right-hand side of Eq. (E.3) we use (DLMF 2013, Eq. (10.32.8))

$K_{v}(z)=\frac{\sqrt{\pi}}{\Gamma(v+1 / 2)}(z / 2)^{v} \int_{1}^{+\infty} \mathrm{d} \gamma\left(\gamma^{2}-1\right)^{v-1 / 2} \exp \{-z \gamma\}$

with Euler's gamma function: $\Gamma(1 / 2+1)=\sqrt{\pi} / 2$.
Callen, J. 2006, Fundamentals of Plasma Physics (Madison: University of Wisconsin)

Cerutti, B., Werner, G. R., Uzdensky, D. A., \& Begelman, M. C. 2012, ApJ, 754, L33

Cerutti, B., Werner, G. R., Uzdensky, D. A., \& Begelman, M. C. 2013, ApJ, 770, 147

Chacón-Acosta, G., Dagdug, L., \& Morales-Técotl, H. A. 2010, Phys. Rev. E, 81,021126

Childs, H., Brugger, E., Whitlock, B., et al. 2012, in High Performance Visualization-Enabling Extreme-Scale Scientific Insight, 357

Cottrill, L. A., Langdon, A. B., Lasinski, B. F., et al. 2008, Phys. Plasmas, 15, 082108

Cubero, D., Casado-Pascual, J., Dunkel, J., Talkner, P., \& Hänggi, P. 2007, Phys. Rev. Lett., 99, 170601

Daughton, W. 2002, Phys. Plasmas, 9, 3668

Daughton, W., Scudder, J., \& Karimabadi, H. 2006, Phys. Plasmas, 13, 072101

Dawson, J. 1962, Phys. Fluids, 5, 445

Dieckmann, M. E. 2009, Plasma Phys. Control. Fusion, 51, 124042

Dieckmann, M. E., Ynnerman, A., Chapman, S. C., Rowlands, G., \& Andersson, N. 2004, Phys. Scr., 69, 456

Dieckmann, M. E., Frederiksen, J. T., Bret, A., \& Shukla, P. K. 2006, Phys. Plasmas, 13, 112110

DLMF 2013, NIST Digital Library of Mathematical Functions, http: //dlmf.nist.gov/, Release 1.0.6 of 2013-05-06

Drake, J. F., Swisdak, M., Che, H., \& Shay, M. A. 2006, Nature, 443, 553

Dunkel, J., \& Hänggi, P. 2009, Phys. Rep., 471, 1

Eldridge, O. C., \& Feix, M. 1962, Phys. Fluids, 5, 1076

Esirkepov, T. Z. 2001, Comput. Phys. Comm., 135, 144

Fitzpatrick, R. 2011, The Phys. Plasmas

Fonseca, R. A., Silva, L. O., Tsung, F. S., et al. 2002, Lect. Notes Comput. Sci., 2331,342

Fonseca, R. A., Martins, S. F., Silva, L. O., et al. 2008, Plasma Phys. Control. Fusion, 50, 124034

Fujimoto, K., \& Machida, S. 2006, J. Comput. Phys., 214, 550

Greenwood, A. D., Cartwright, K. L., Luginsland, J. W., \& Baca, E. A. 2004, provided by the SAO/NASA Astrophysics Data System, J. Comput. Phys., 201, 665,

Grosskopf, M., Drake, R., Kuranz, C., et al. 2013, High Energy Density Physics, 9, 192

Grote, D. P., Friedman, A., Vay, J.-L., \& Haber, I. 2005, in Electron Cyclotron Resonance Ion Sources, ed. M. Leitner, Am. Ins. Phys. Conf. Ser., 749, 55

Hartree, D. R. 1950, Appl. Sci. Res., 1, 379

Haugboelle, T., Fredriksen, J. T., \& Nordlund, A. 2013, Phys. Plasmas, 20, 062904-062904-24

Hededal, C. 2005, Ph.D. Thesis, Niels Bohr Institute

Hesse, M., Kuznetsova, M., \& Birn, J. 2001, J. Geophys. Res., 106, 29831

Hockney, R. W. 1966, Phys. Fluids, 9, 1826

Hockney, R. W. 1971, J. Comput. Phys., 8, 19

Hockney, R. W., \& Eastwood, J. W. 1988, Computer simulation using particles (Institute of Physics Publishing)

Hoh, F. C. 1966, Phys. Fluids, 9, 277

Huang, J., \& Ma, Z.-W. 2008, Chin. Phys. Lett., 25, 1764

Jaroschek, C. H., \& Hoshino, M. 2009, Phys. Rev. Lett., 103, 075002

Jaroschek, C. H., Lesch, H., \& Treumann, R. A. 2005, ApJ, 618, 822

Jüttner, F. 1911, Ann. Phys., 339, 856

Kirk, J. G., \& Skjæraasen, O. 2003, ApJ, 591, 366

Klimas, A., Hesse, M., \& Zenitani, S. 2008, Phys. Plasmas, 15, 082102

Klimontovich, I. L. 1982, Oxford Pergamon Press International Series on Natural Philosophy, 105

Langdon, A. B. 1970, J. Comput. Phys., 6, 247

Markidis, S., Lapenta, G., \& Rizwan-uddin 2010, Math. Comput. Simul., 80, 1509

Markidis, S., Henri, P., Lapenta, G., et al. 2012, Nonlinear Processes in Geophysics, 19, 145

Matsumoto, H., \& Omura, Y. 1993, Computer Space Plasma Physics: Simulation Techniques and Software

Medvedev, M. V., Fiore, M., Fonseca, R. A., Silva, L. O., \& Mori, W. B. 2005, ApJ, 618, L75

Messmer, P. 2001, Ph.D. Thesis, Institute of Astronomy, ETH Zürich, Switzerland, No. 14412

Messmer, P. 2002, A\&A, 382, 301

Michno, M. J., \& Schlickeiser, R. 2010, ApJ, 714, 868

Mihalas, D., \& Mihalas, B. W. 1984, Foundations of radiation hydrodynamics (New York: Oxford University Press)

Nicholson, D. R. 1983, Introduction to Plasma Theory, eds. John Wiley \& Sons

Nieter, C., \& Cary, J. R. 2004, J. Comput. Phys., 196, 448 
Nishikawa, K.-I., Mizuno, Y., Fishman, G. J., \& Hardee, P. 2008, Int. J. Mod. Phys. D, 17, 1761

Nishikawa, K.-I., Niemiec, J., Medvedev, M., et al. 2011, in AIP Conf. Ser. 1366, eds. V. Florinski, J. Heerikhuisen, G. P. Zank, \& D. L. Gallagher, 163

Paesold, G., Blackman, E. G., \& Messmer, P. 2005, Plasma Phys. Control. Fusion, 47, 1925

Pétri, J., \& Kirk, J. G. 2007, Plasma Phys. Control. Fusion, 49, 1885

Pétri, J., \& Lyubarsky, Y. 2007, A\&A, 473, 683

Pomraning, G. C. 1973, The equations of radiation hydrodynamics (Pergamon Press)

Silva, L. O., Fonseca, R. A., Tonge, J. W., et al. 2003, ApJ, 596, L121

Sironi, L., \& Spitkovsky, A. 2011, ApJ, 726, 75

Spitkovsky, A. 2005, in Astrophysical Sources of High Energy Particles and Radiation, eds. T. Bulik, B. Rudak, \& G. Madejski, Am. Inst. Phys. Conf. Ser., 801,345
Spitzer, L. 1965, Physics of fully ionized gases (New York: Interscience Publication)

Swisdak, M. 2013, Phys. Plasmas, 20, 062110-062110-4

Tien, P. K., \& Moshman, J. 1956, J. Appl. Phys., 27, 1067

Treumann, R. A., Nakamura, R., \& Baumjohann, W. 2011, Ann. Geophys., 29, 1259

Trier Frederiksen, J., Haugbølle, T., Medvedev, M. V., \& Nordlund, Å. 2010, ApJ, 722, L114

Vay, J.-L. 2008, Phys. Plasmas, 15, 056701

Whitlock, B., Favre, J. M., \& Meredith, J. 2011, In Proc. of the 11th Eurographics Symposium on Parallel Graphics and Visualization (EGPGV'11), 101

Yu, S. P., Kooyers, G. P., \& Buneman, O. 1965, J. Appl. Phys., 36, 2550

Zenitani, S., \& Hoshino, M. 2008, ApJ, 677, 530 